\title{
Number of Distinct Homomorphic Images in Coset Diagrams
}

\author{
Muhammad Aamir $\mathbb{D},{ }^{1}$ Muhammad Awais Yousaf $\mathbb{D},{ }^{1}$ and Abdul Razaq $\mathbb{D}^{2}$ \\ ${ }^{1}$ Department of Mathematics, The Islamia University of Bahawalpur, Bahawalpur 63100, Pakistan \\ ${ }^{2}$ Division of Science and Technology, Department of Mathematics, University of Education, Lahore 54000, Pakistan
}

Correspondence should be addressed to Muhammad Aamir; aamir.math.hed@gmail.com

Received 12 December 2020; Accepted 28 March 2021; Published 2 August 2021

Academic Editor: Elena Guardo

Copyright (c) 2021 Muhammad Aamir et al. This is an open access article distributed under the Creative Commons Attribution License, which permits unrestricted use, distribution, and reproduction in any medium, provided the original work is properly cited.

\begin{abstract}
The representation of the action of PGL $(2, \mathbb{Z})$ on $F_{t} \cup\{\infty\}$ in a graphical format is labeled as coset diagram. These finite graphs are acquired by the contraction of the circuits in infinite coset diagrams. A circuit in a coset diagram is a closed path of edges and triangles. If one vertex of the circuit is fixed by $(p q)^{\Delta_{1}}\left(p q^{-1}\right)^{\Delta_{2}}(p q)^{\Delta_{3}} \ldots\left(p q^{-1}\right)^{\Delta_{m}} \in \operatorname{PSL}(2, \mathbb{Z})$, then this circuit is titled to be a length- $m$ circuit, denoted by $\left(\Delta_{1}, \Delta_{2}, \Delta_{3}, \ldots, \Delta_{m}\right)$. In this manuscript, we consider a circuit $\Delta$ of length 6 as $\left(\Delta_{1}, \Delta_{2}, \Delta_{3}, \Delta_{4}, \Delta_{5}, \Delta_{6}\right)$ with vertical axis of symmetry, that is, $\Delta_{2}=\Delta_{6}, \Delta_{3}=\Delta_{5}$. Let $\Gamma_{1}$ and $\Gamma_{2}$ be the homomorphic images of $\Delta$ acquired by contracting the vertices $a, u$ and $b, v$, respectively, then it is not necessary that $\Gamma_{1}$ and $\Gamma_{2}$ are different. In this study, we will find the total number of distinct homomorphic images of $\Delta$ by contracting its all pairs of vertices with the condition $\Delta_{1}>\Delta_{2}>\Delta_{3}>\Delta_{4}$. The homomorphic images are obtained in this way having versatile applications in coding theory and cryptography. One can attain maximum nonlinearity factor using this in the encryption process.
\end{abstract}

\section{Introduction}

It is prominent that the finite presentation $\left\langle p, q ; p^{2}=q^{3}=\right.$ $1\rangle$ is known as the modular group $\operatorname{PSL}(2, \mathbb{Z})$ generated by the linear fractional transformations $p: \chi \longrightarrow(-1 / \chi)$ and $q: \chi \longrightarrow((\chi-1) / \chi)$. In [1], Akbas discussed suborbitalgraphs for the modular group by showing that these graphs contains no circuit if and only if it contains no triangles. If we insert an extra generator $r=(1 / \chi)$ with $p$ and $q$, another group is emerged, denoted as $\operatorname{PGL}(2, \mathbb{Z})[2]$, an extension of $\operatorname{PSL}(2, \mathbb{Z})$ with the finite presentation as

$$
\left\langle p, q, r ; p^{2}=q^{3}=r^{2}=(p r)^{2}=(q r)^{2}=1\right\rangle .
$$

In 1978, Professor Graham Higman propounds an unfamiliar type of a graph, titled as coset diagram, which presents the action of PGL $(2, \mathbb{Z})$ on $\operatorname{PL}\left(F_{t}\right)$, where $F_{t}$ is a finite field and $t$ shows a prime power. In 1983 , this foundation is laid by Qaisar Mushtaq [3]. Small triangles are proposed for the cycle $q^{3}$, such that $q$ permutes the vertices of triangles in the opposite direction of rotation of clock and an edge is attached to any two vertices that are interchanged by $p$. Heavy dots represent the fixed points of $p$ and $q$. Note that $(p r)^{2}=1$ equals $r q r=q^{-1}$, which means $r$ reverses the triangle orientation proposed for the cycle $q^{3}$. For that reason, the diagram need not to be made more perplexing by interjecting $r$ edges.

A coset diagram (subdiagram) $\Gamma_{1}$ is said to be a homomorphic image of the coset diagram (subdiagram) $\Gamma_{2}$ if and only if $\left|V\left(\Gamma_{1}\right)\right|<\left|V\left(\Gamma_{2}\right)\right| \forall a \in V\left(\Gamma_{2}\right)$ with $(a) h=a$, where $h \in \operatorname{PSL}(2, \mathbb{Z})$, there exist a vertex $u$ in $V\left(\Gamma_{1}\right)$ such that $(u) h=u$.

Coset diagrams obtained from the action of $\operatorname{PSL}(2, \mathbb{Z})$ over $Q_{\epsilon}$ are infinite graphs [4], where $Q_{\varepsilon}$ $=\left\{a_{1}+a_{2} \sqrt{\varepsilon} ; a_{1}, a_{2} \in \mathbb{Q}\right.$ and $\varepsilon \in \mathbb{Z}^{+}$is a square free $\}$. These diagrams are not easy to study because they are infinite. Thence, coset diagrams are considered as important for the action of $\operatorname{PSL}(2, \mathbb{Z})$ on $\operatorname{PL}\left(F_{q}\right)$ because this action presents finite graphs. The number $\left(\left(x_{1}+\sqrt{\varepsilon}\right) / x_{2}\right)$ is an expression of the number $a_{1}+a_{2} \sqrt{\varepsilon} \in Q_{\varepsilon}$, where $\left(x_{1}, x_{2},\left(\left(x_{1}^{2}-\varepsilon\right) / x_{2}\right)\right)=$ 1 . These finite coset diagrams are the homomorphic images of the coset diagrams for $\left(\left(x_{1}+\sqrt{\varepsilon}\right) / x_{2}\right)$, where $\varepsilon \equiv z^{2} \bmod p$ for some $z \in N$.

To explain more, coset diagram in Figure 1 illustrates the action on $\operatorname{PL}\left(F_{17}\right)$ by $\operatorname{PGL}(2, \mathbb{Z})$ with permutation 


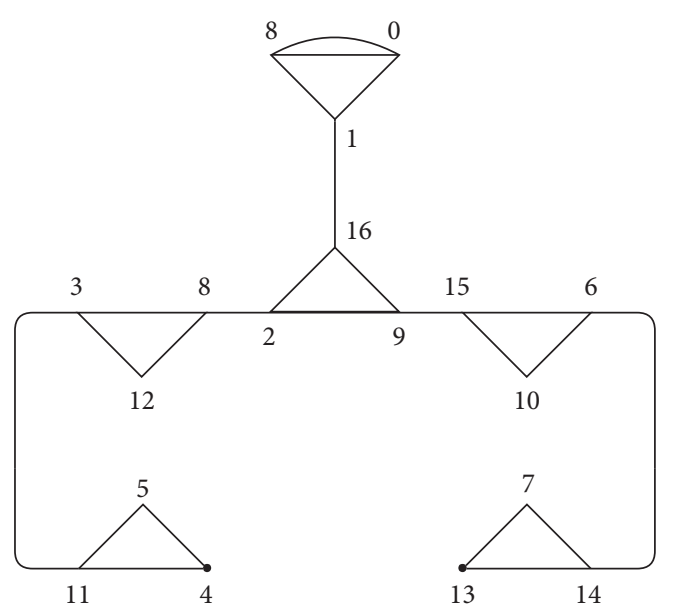

Figure 1: Coset diagram for the action of $\operatorname{PGL}(2, \mathbb{Z})$ on $\operatorname{PL}\left(F_{17}\right)$.

representations $p, q$, and $r$ by $(\chi) p=(-1 / \chi), \quad(\chi) q=$ $((\chi-1) / \chi)$, and $(\chi) r=(1 / \chi)$, respectively, as

$p$ : (0œ), (1 16), (2 8), (3 11), (4), (5 10), (6 14), (7 12),

(9 15), (13),

$q$ : (0œ 1), (2916), (3 12 8), (4 511$),(61510),(71314)$,

$r:(0 \infty),(1),(29),(36),(413),(57),(815),(1012)$,

(11 14), (16).

Thus, $13=8^{2} \bmod 17$ gives that the coset diagram $\operatorname{PGL}(2,17)$ is a homomorphic image of the coset diagram for $\left(\left(x_{1}+\sqrt{13}\right) / x_{2}\right)$.

Coset diagrams obtained from the action of $\operatorname{PSL}(2, \mathbb{Z})$ on $\mathbb{Q}_{\varepsilon}$ have some attractive narrative. In [5], the quadratic irrational numbers are classified by taking prime modulus that proved helpful in investigating the modular group action on the real quadratic field. The number $\bar{B}=\left(\left(x_{1}-\right.\right.$ $\left.\sqrt{\varepsilon}) / x_{2}\right)$ is called the conjugate of $B=\left(\left(x_{1}+\sqrt{\varepsilon}\right) / x_{2}\right)$, where $x_{1}$ and $x_{2}$ are integers and $\varepsilon$ is a fixed number from $\mathbb{Z}^{+}$, which is not a perfect square. $B$ is said to be an ambiguous number [6], if the sign of $B$ is different from the sign of $\bar{B} . B$ is said to be a totally negative (positive) if $B$ and $\bar{B}$ both have the same signs. For a fixed $\epsilon$, the number of ambiguous numbers of the form $B=\left(\left(x_{1}+\sqrt{\varepsilon}\right) / x_{2}\right)$ is finite and that segment of the coset diagram attained by the ambiguous numbers forms a closed path (circuit) and it is the only closed path in $B$-orbit [4]. With the help of coset diagram, Anna Torstensson not only described the applications to study the finitely presented group but also discussed the one-relator quotients of the modular group [7].

A closed path of triangles and edges in a coset diagram is called a circuit. In a coset diagram, a circuit is said to be a length- $k$ circuit, denoted by $\left(\Delta_{1}, \Delta_{2}, \Delta_{3}, \ldots, \Delta_{k}\right)$, if its one vertex is fixed by

$$
(a x)^{\Delta_{1}}\left(a x^{-1}\right)^{\Delta_{2}}(a x)^{\Delta_{3}} \ldots\left(a x^{-1}\right)^{\Delta_{k}} \in \operatorname{PSL}(2, \mathbb{Z}) .
$$

Alternatively, it means that one vertex of the $\Delta_{1}$ triangles lies outside of the circuit and one vertex of the $\Delta_{2}$ triangles lies inside of the circuit and likewise. Since $\left(\Delta_{1}, \Delta_{2}, \Delta_{3}, \ldots, \Delta_{k}\right)$ is a cycle, so it does not matter if one vertex of the $\Delta_{1}$ triangles lies inside of the circuit and one vertex of the $\Delta_{2}$ triangles lies outside of the circuit and likewise. Note that $k$ is always even.

The circuit of the type $\left(\Delta_{1}\right.$, $\left.\Delta_{2}, \Delta_{3}, \ldots, \Delta_{l^{\prime}}, \Delta_{1}, \Delta_{2}, \Delta_{3}, \ldots, \Delta_{l^{\prime}}, \ldots, \Delta_{1}, \Delta_{2}, \Delta_{3}, \ldots, \Delta_{l^{\prime}}\right)$ is termed as a periodic circuit with period of length $l$.

Note 1. By $V(\Delta)$, we mean the collection of vertices lies on the circuit $\Delta=\left(\Delta_{1}, \Delta_{2}, \Delta_{3}, \ldots, \Delta_{k}\right)$.

Let $a, u \in V(\Delta)$ be any two vertices fixed by the words $h_{1}$ and $h_{2}$, that is, $(a) h_{1}=a$ and $(u) h_{2}=u$. Suppose $h_{3}$ is the word that maps $a$ to $u$, then $(a) h_{1}^{-1} h_{3}=u$. Note that $h_{3}$ and $h_{1}^{-1} h_{3}$ are the only two paths that assign $a$ to $u$. Now, by contraction of the pair of vertices $a$ and $u$, we mean that $a$ and $u$ melt together to become one node $s=a=u$ such that $(s) h_{3}=(s) h_{1}^{-1} h_{3}=s$. As a result of this contraction, a closed path $\Gamma$ is created that contains the vertex $s$ fixed by $h_{3}$ and $h_{1}^{-1} h_{3}$. This closed path $\Gamma$ is the homomorphic image of the circuit $\Delta$. It is important to note that $a$ and $u$ is not the only pair of contraction in $\Delta$ that creates homomorphic image $\Gamma$. There are also many pairs of contraction other than $a$ and $u$ that create the same homomorphic image $\Gamma$. The following theorems proved in [8] will help us to find the total number of such contracted pairs that produce the same homomorphic image $\Gamma$ of $\Delta$.

Theorem 1. Let the vertices $a$ and $u$ in $\Delta$ are contracted and $a$ homomorphic image $\Gamma$ of $\Delta$ is evolved, then $\Gamma$ is also obtainable if the vertices $(a) h$ and $(u) h$ in $\Delta$ are contracted.

Theorem 2. If $a$ and $u$ are contracted to obtain $\Gamma$, then during this process $|E|$ number of pairs are contracted all together, where $E$ is the collection of words such that $\forall h \in E,(a) h$ and $(u) h$ are contained by $\Delta$.

Example 1. Let us contract the vertices $a$ and $u$ from the circuit $(3,4,2,4,2,4)$ (Figure 2) and acquire a homomorphic $\Gamma$ (Figure 3 ) of the circuit $(3,4,2,4,2,4)$. Thus, $h_{1}=q(p q)$ and $h_{2}=(p q)\left(p q^{-1}\right)^{4}(p q)^{2}\left(p q^{-1}\right)^{4}(p q)^{2}\left(p q^{-1}\right)^{4} p$ are the two possible paths between $a$ and $u$ that are fixing the vertex $s=a=u$ in $\Gamma$.

Let $E$ be the family of words such that for all $h \in E$ implies $(a) h$ and $(u) h$ lie on the circuit $(3,4,2,4,2,4)$, then $E=\left\{e, q, q^{-1}, p, p q, p q^{-1},(p q) p,(p q)^{2},(p q) p q^{-1}\right\}$. Then by Theorem 2 , the cardinality of $E$ implies that there are 9 pairs of vertices contracted to generate the homomorphic image $\Gamma$.

Note that the cardinality of $E$ does not give the total number of contracted pairs to generate the homomorphic image $\Gamma$. In the following, we will discuss the process to find the total number of contracted pairs to generate $\Gamma$.

Let $\Gamma^{*}$ denote itself as the mirror image of $\Gamma$. Thus, the permutation $r$ ensures that the coset diagram is symmetric along the vertical axis. This implies $\Gamma^{*}$ will assuredly occur.

If $u=p q^{\pi_{1}} p q^{\pi_{2}} \ldots p q^{\pi_{n}}\left(\pi_{i}=1\right.$ or -1$)$ is a word, then $u^{*}=p q^{-\pi_{1}} p q^{-\pi_{2}} \ldots p q^{-\pi_{n}}$. If the word $h$ fixes the vertex $s$, then the vertex $s^{*}$ is fixed by $u^{*}$. 


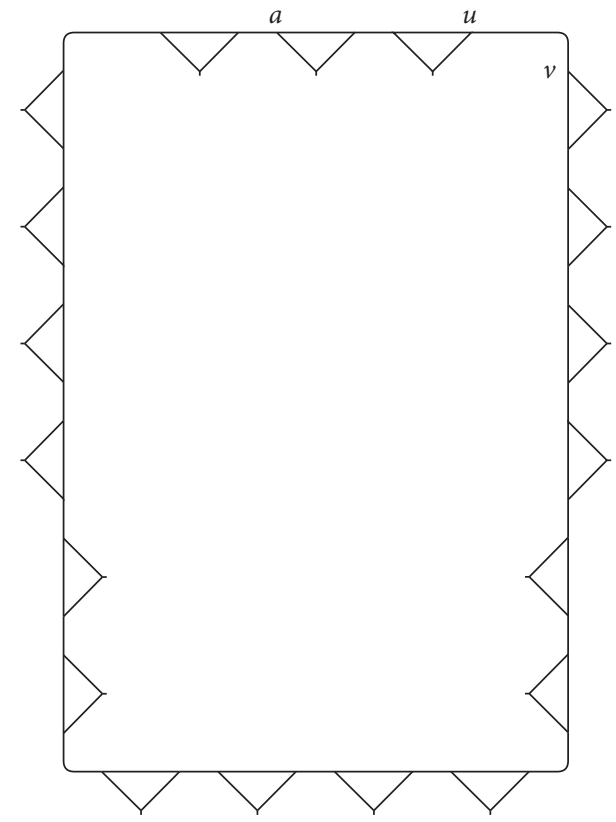

Figure 2: Circuit $(3,4,2,4,2,4)$.

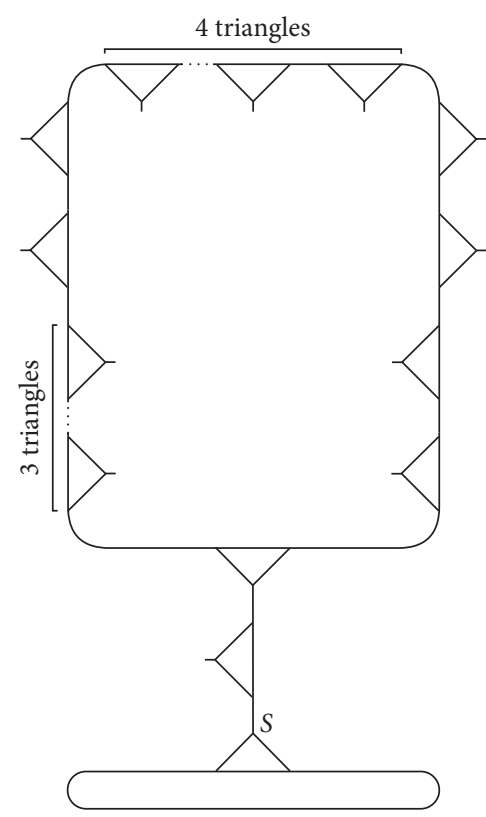

FIgURE 3: Homomorphic image $\Gamma$.

A homomorphic image $\Gamma$ has a symmetry with respect to vertical axis if and only if by contracting $a$ and $u$, the vertices $a^{*}$ and $u^{*}$ are also contracted.

Remark 1. In coset diagrams, $r$ reverses the orientation of the triangles representing the three cycles of $q$ (as reflection does). So corresponding to each vertex $s$ fixed by the pair $h_{1}$, $h_{2}$, there is a vertex $s^{*}$ in $\Gamma^{*}$ (mirror image of $\Gamma$ ) such that $s^{*}$ is a fixed point of $h_{1}^{*}, h_{2}^{*}$. In other words, it is created by contracting $a^{*}$ and $u^{*}$. There are certain $\Gamma^{\prime}$ 's which have a vertical symmetry and so have the same orientations as those of their mirror images. The homomorphic image $\Gamma$ of a circuit $\Delta=\left(\Delta_{1}, \Delta_{2}, \Delta_{3}, \Delta_{4}, \Delta_{5}, \Delta_{6}\right)$, which has a vertex $s$ fixed by the pair $h_{1}, h_{2}$, has the same orientation as that of its mirror image if and only if there is a vertex $s^{*}$ in $\Gamma$ such that $\left(s^{*}\right) h_{1}=\left(s^{*}\right) h_{2}=s^{*}$.

1.1. Counting the Number of Pairs of Contracting Vertices of a Homomorphic Image. Let $\Gamma$ be a homomorphic image of the circuit $\Delta$ acquired by the contraction of pair of vertices $a$ and $u$ of $\Delta$. Then by Theorem $2, \Gamma$ has $|E|$ number of pairs of vertices. To find the total number of pairs of vertices, one should follow the following steps.

To know how many total pairs of contracting vertices are there, special precaution must be taken.

(1) If by contracting $a$ and $u$ to create $\Gamma$, the pair of vertices $a^{*}$ and $u^{*}$ are not contracted, then $\Gamma$ has different orientation from its mirror image $\Gamma^{*}$. So, there are $|E|$ number of more pairs of vertices for the mirror image of $\Gamma$.

(2) If by contracting $a$ and $u$ to create $\Gamma$, the pair of vertices $a^{*}$ and $u^{*}$ are also contracted, then $\Gamma$ has the same orientation as that of its mirror image $\Gamma^{*}$. So, in this case, $\Gamma$ has $|E|$ number of pairs of contracted vertices.

Consider a circuit of length 6 as $\Delta=\left(\Delta_{1}, \Delta_{2}, \Delta_{3}, \Delta_{4}, \Delta_{5}, \Delta_{6}\right)$ (Figure 4$)$ with vertical axis of symmetry, that is, $\Delta_{2}=\Delta_{6}, \Delta_{3}=\Delta_{5}$. Suppose $\Delta_{1}>\Delta_{2}>\Delta_{3}>\Delta_{4}$. The coset diagrams are composed of circuits. The vertices of the circuits in infinite diagrams are contracted in a certain way, and a finite coset diagram evolves. It is therefore necessary to ask how many distinct homomorphic images are obtained if we contract all the pairs of vertices of the circuit $\Delta$ ? We not only give the answer to this question for a circuit $\Delta$ but also mention those pairs of vertices which are "important". There is no need to contract the pairs which are not mentioned as "important". If we contract those, we obtain a homomorphic image, which we have already obtained by contracting “important" pairs.

Note 2. It is clear from Figure 4 that

(1) The mirror image of the vertex $e_{l}^{k}$ is $e^{\frac{2-k}{3 \Delta_{k}}-(l-1)}$, that is, $e_{l}^{k *}=e_{3 \Delta_{k}-(l-1)}^{\frac{2-k}{2}}$

(2) The vertex $a_{3 m+1}^{k}$ is fixed by the word $\left(p q^{-1}\right)^{m}(p q)$ $\Delta_{\underline{k} \pm 5}\left(p q^{-1}\right)$

$\underline{\Delta_{k \pm 4}}(p q)^{\Delta_{k \pm 3}}\left(p q^{-1}\right)^{\Delta_{k \pm 2}}(p q)^{\Delta_{k \pm 1}}\left(p q^{-1}\right)^{\Delta_{k}-m}$

(3) The vertex $x_{3 m}^{k}$ is fixed by the word (pq) $\Delta_{k}-m\left(p q^{-1}\right)^{\Delta}$

$\underline{k \pm 1}(p q)^{\Delta_{k \pm 2}}\left(p q^{-1}\right)^{\Delta_{k \pm 3}}(p q)^{\Delta_{k \pm 4}}\left(p q^{-1}\right)^{\Delta_{k \pm 5}}(p q)^{m}$, where

$k \in\{1,2,3,4,5,6\} ; l=1,2,3, \ldots, \Delta_{k} ; m=$

$1,2,3, \ldots, \Delta_{k}-1, \underline{n}= \begin{cases}6, & \text { if } n=0,6 \\ n(\bmod 6) & \text { otherwise }\end{cases}$

and $\pm=\left\{\begin{array}{ll}+ & \text { if } k \text { is odd } \\ - & \text { otherwise }\end{array}\right.$. 


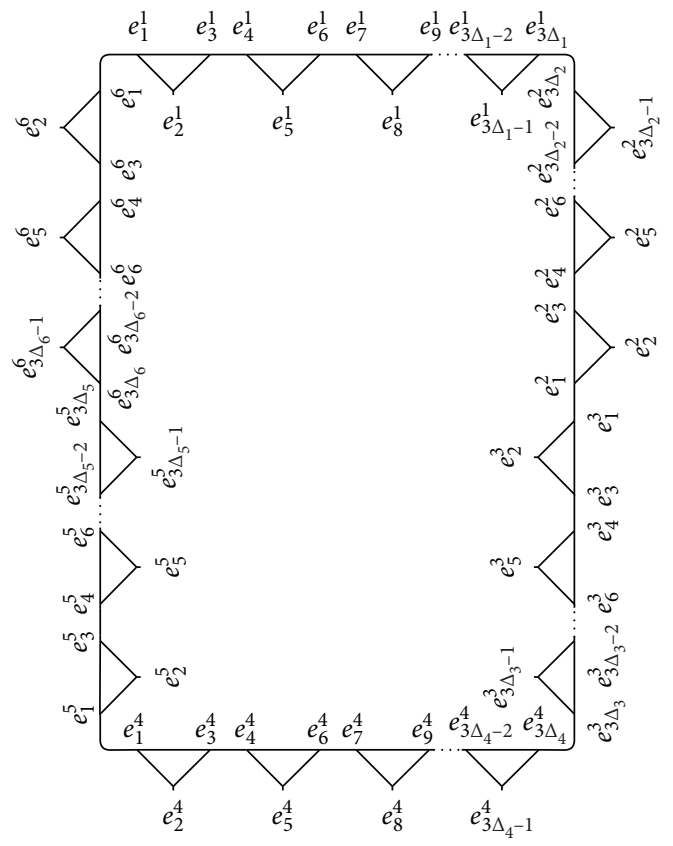

Figure 4: The circuit $\Delta=\left(\Delta_{1}, \Delta_{2}, \Delta_{3}, \Delta_{4}, \Delta_{5}, \Delta_{6}\right)$.

Lemma 1. If we contract the vertices $e_{3 l_{1}+1}^{1}: l_{1}=0,1,2, \ldots$, $\Delta_{2}-1$, with the vertex $e_{3 \Delta_{1}}^{1}$ in the circuit $\Delta$, then there arise $\Delta_{2}$ distinct homomorphic images of $\Delta$ and $3\left(l_{1}+2\right)$ pairs of contracted vertices create each homomorphic image. Moreover, the number of total pairs of contracted vertices to generate all $\Delta_{2}$ and their mirror homomorphic images of $\Delta$ is $3\left(\Delta_{2}^{2}+3 \Delta_{2}-2\right)$.

Proof. Let $H_{1}=\left\{\Omega_{l_{1}}^{1} ; l_{1}=0,1,2, \ldots, \Delta_{2}-1\right\}$ (Figure 5) be the collection of homomorphic images of $\Delta$ acquired by the contraction of the vertices $e_{3 l_{1}+1}^{1}: l_{1}=0,1,2, \ldots, \Delta_{2}-1$ with the vertex $e_{3 \Delta_{1}}^{1}$ in $\Delta$, where $e_{3 l_{1}+1}^{1}$ and $e_{3 \Delta_{1}}^{1}$ are fixed by the words $\quad(p q)^{l_{1}}\left(p q^{-1}\right)^{\Delta_{6}}(p q)^{\Delta_{5}}\left(p q^{-1}\right)^{\Delta_{4}}(p q)^{\Delta_{3}} \quad\left(p q^{-1}\right)^{\Delta_{2}}$ $(p q)^{\Delta_{1}-l_{1}}$ and $(p q)^{\Delta_{2}}\left(p q^{-1}\right)^{\Delta_{3}}(p q)^{\Delta_{4}}\left(p q^{-1}\right)^{\Delta_{5}}(p q)^{\Delta_{6}}$ $\left(p q^{-1}\right)^{\Delta_{1}}$. It is easy to verify that $P_{1}=q^{-1}\left(p q^{-1}\right)^{\Delta_{1}-l_{1}-1}$ and $P_{2}=(p q)^{\Delta_{2}}\left(p q^{-1}\right)^{\Delta_{3}}(p q)^{\Delta_{4}}\left(p q^{-1}\right)^{\Delta_{5}}(p q)^{\Delta_{6}}\left(p q^{-1}\right)^{1_{1}} p$ are the possible paths between $e_{3 l_{1}+1}^{1}$ and $e_{3 \Delta_{1}}^{1}$ (Figure 4). This implies that for each $l_{1}$, the homomorphic image $\Omega_{l_{1}}^{1}$ has a vertex $s$ fixed by $P_{1}$ and $P_{2}$.

Thus,

$$
\begin{aligned}
E_{1}= & \left\{e, q, q^{-1}, p, p q, p q^{-1}, p q p,(p q)^{2}, p q p q^{-1}, \ldots,\right. \\
& \left.(p q)^{l_{1}} p,(p q)^{l_{1}+1},(p q)^{l_{1}} p q^{-1}\right\}
\end{aligned}
$$

is the family of elements in $\operatorname{PSL}(2, \mathbb{Z})$ such that $\forall x \in E, \Delta$ contains the vertices $\left(e_{3 l_{1}+1}^{1}\right) x$ and $\left(e_{3 \Delta_{1}}^{1}\right) x$. This gives that the cardinality of $E_{1}$, that is, $3\left(l_{1}+2\right)$ is the number of contracted pairs of vertices to produce the homomorphic image $\Omega_{l_{1}}^{1}$ (Theorem 2). For $m \neq n$, let $\Omega_{m}^{1}$ and $\Omega_{n}^{1}$ be any two elements of $H_{1}$, then the number of triangles in $\Omega_{m}^{1}$ and $\Omega_{n}^{1}$ are not equal (Figure 5). This implies that all the elements in $H_{1}$ are different and no one is the mirror image of the other. This

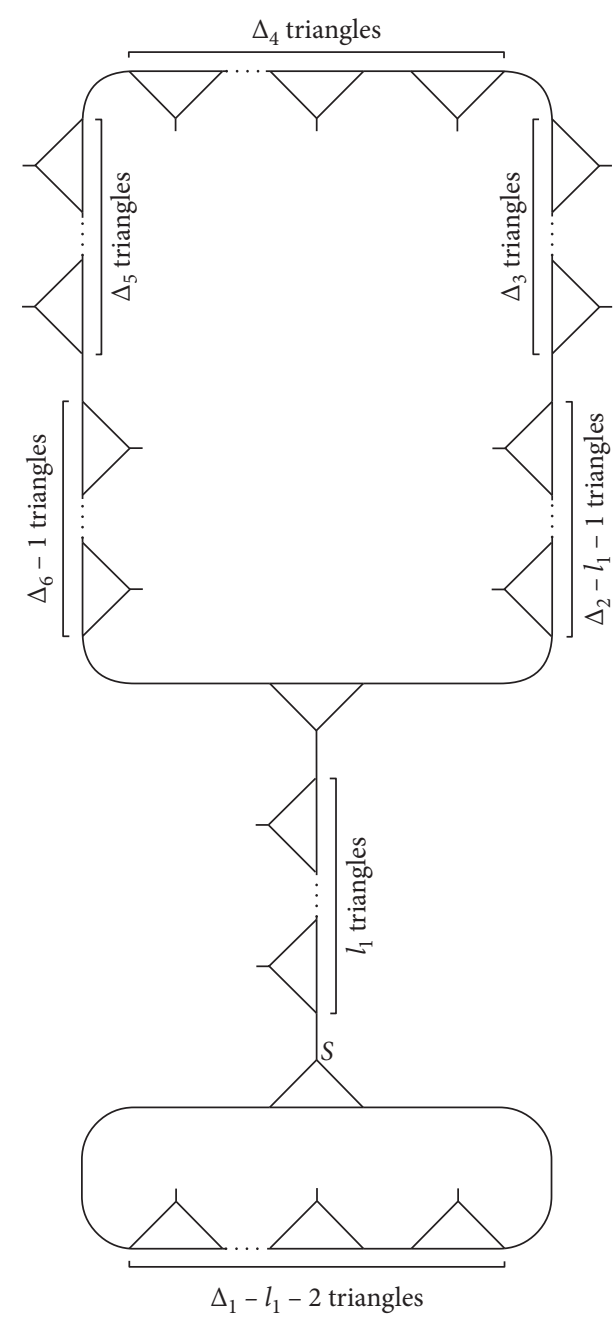

Figure 5: Homomorphic images $\Omega_{l_{1}}^{1}$.

further forms the result as $\left|H_{1}\right|=\Delta_{2}$. Thence, the number of contracted pairs of vertices of $\Delta$ to create all the elements of $H_{1}$ is $\sum_{l_{1}=0}^{\Delta_{2}-1} 3\left(l_{1}+2\right)$.

From Figure 5, it is also clear that no element of $H_{1}$ except $\Omega_{0}^{1}$ has vertical axis of symmetry. So, $\Omega_{0}^{1}$ is the only homomorphic image whose orientation is not different from its mirror image $\Omega_{0}^{1 *}$ and all the remaining $\Delta_{2}-1$ elements of $H_{1}$ have different orientations from their mirror images. Hence, there are

$$
6 \sum_{l_{1}=1}^{\Delta_{2}-1}\left(l_{1}+2\right)+6=3\left(\Delta_{2}^{2}+3 \Delta_{2}-2\right)
$$

pairs of contracted vertices to produce all the homomorphic images in $H_{1}$.

Lemma 2. If we contract the vertices $e_{3 l_{1}+1}^{2}: l_{1}=0,1,2, \ldots$, $\Delta_{2}-1$, with the vertex $e_{3 \Delta_{1}}^{1}$ in the circuit $\Delta$, then there arise $\Delta_{2}$ distinct homomorphic images of $\Delta$ and $3\left(l_{1}+2\right)$ pairs of contracted vertices create each homomorphic image. Moreover, the number of total pairs of contracted vertices to generate all $\Delta_{2}$ and their mirror homomorphic images of $\Delta$ is $3\left(\Delta_{2}^{2}+3 \Delta_{2}\right)$. 
Let $H_{2}=\left\{\Omega_{l_{1}}^{2} ; l_{1}=0,1,2, \ldots, \Delta_{2}-1\right\}$ be the collection of homomorphic images of $\Delta$ acquired by the contraction of the vertices $e_{3 l_{1}+1}^{2}: l_{1}=0,1,2, \ldots, \Delta_{2}-1$ with the vertex $e_{3 \Delta_{1}}^{1}$ in $\Delta$. Suppose $r$ shows itself as the remainder of $\left(l_{1} /\left(\Delta_{2}-l_{1}\right)\right)$. Then, graphically we make four partitions of $\mathrm{H}_{2}$ as follows:

(i) $\Omega_{l_{1}}^{2}: \Delta_{2}-2 l_{1}>1$ (Figure 6(a))

(ii) $\Omega_{l_{1}}^{2}: \Delta_{2}-2 l_{1}=1$ (Figure 6(b))

(iii) $\Omega_{l_{1}}^{2}: \Delta_{2}-2 l_{1}<1$ and $\Delta_{2}-l_{1}>r+1$ (Figure 6(c))

(iv) $\Omega_{l_{1}}^{2}: \Delta_{2}-2 l_{1}<1$ and $\Delta_{2}-l_{1}=r+1$ (Figure 6(d))

From all the homomorphic images presented in these figures, it is not intricated to check that no one is the mirror image of itself. This lemma can be proved by using the same procedure as that for Lemma 1.

Let $k_{1} \in\{3,4,5\}$.

Lemma 3. If we contract the vertices $e_{3 l_{\left(2, k_{1}+1\right.}+k_{1}}^{k_{1}} l_{\left(2, k_{1}\right)}=$ $0,1,2, \ldots, \Delta_{k_{1}}-1$, with the vertex $e_{3 \Delta_{1}}^{1}$ in the circuit $\Delta$, then for each $k_{1}$, there arise $\Delta_{k_{1}}$ distinct homomorphic images of $\Delta$ and $3\left(l_{\left(2, k_{1}\right)}+2\right)$ pairs of contracted vertices that create each homomorphic image. Moreover, the number of total pairs of contracted vertices to generate all $\Delta_{k_{1}}$ and their mirror homomorphic images of $\Delta$ is $3\left(\Delta_{k_{1}}^{2}+3 \Delta_{k_{1}}\right)$.

For a fix value of $k_{1}$, let $H_{3}^{k_{1}}=\left\{\Omega_{l_{\left(2, k_{1}\right)}^{3}} ; l_{\left(2, k_{1}\right)}=\right.$ $\left.0,1,2, \ldots, \Delta_{k_{1}}-1\right\}$ be the collection of homomorphic images of $\Delta$ acquired by the contraction of the vertices $e_{3 l_{\left(2, k_{1}\right)+1}^{k_{1}}:}: l_{\left(2, k_{1}\right)}=0,1,2, \ldots, \Delta_{k_{1}}-1$, with the vertex $e_{3 \Delta_{1}}^{1}$ in $\Delta$. Figures 7-9 present $H_{3}^{k_{1}}$ graphically. From all the homomorphic images presented in these figures, it is not intricated to check that no one is the mirror image of itself.

This lemma can be proved by using the same procedure as that for Lemma 1 .

Lemma 4. If we contract the vertices $e_{3 l_{3}+1}^{6}: l_{3}=0,1,2, \ldots$, $\Delta_{6}-1$, with the vertex $e_{3 \Delta_{1}}^{1}$ in the circuit $\Delta$, then there arise $\Delta_{6}$ distinct homomorphic images of $\Delta$ and $3\left(l_{3}+2\right)$ pairs of contracted vertices create each homomorphic image. Moreover, the number of total pairs of contracted vertices to generate all $\Delta_{6}$ and their mirror homomorphic images of $\Delta$ is $(3 / 2)\left(\Delta_{6}^{2}+3 \Delta_{6}\right)$.

Let $H_{4}=\left\{\Omega_{l_{3}}^{4} ; l_{3}=0,1,2, \ldots, \Delta_{6}-1\right\}$ be the collection of homomorphic images of $\Delta$ acquired by the contraction of the vertices $e_{3 l_{6}+1}^{1}: l_{3}=0,1,2, \ldots, \Delta_{6}-1$ with the vertex $e_{3 \Delta_{1}}^{1}$ in $\Delta$. Figure 10 presents $H_{4}$ graphically. From all the homomorphic images presented in Figure 10, it is not intricated to check that every one is the mirror image of itself.

This lemma can be proved by using the same procedure as that for Lemma 1.

Lemma 5. If we contract the vertices $e_{3 l_{4}+1}^{1}: l_{4}=1,2, \ldots$, $\Delta_{1}-1$, with the vertex $e_{3 \Delta_{2}}^{2}$ in the circuit $\Delta$, then there arise $\Delta_{1}-1$ distinct homomorphic images of $\Delta$ and $3\left(l_{4}+2\right)$ pairs of contracted vertices create each homomorphic image. Moreover, the number of total pairs of contracted vertices to generate all $\Delta_{1}-1$ and their mirror homomorphic images of $\Delta$ is $(3 / 2)\left(\Delta_{1}^{2}+3 \Delta_{1}-4\right)$.

Let $H_{5}=\left\{\Omega_{l_{4}}^{5} ; l_{4}=1,2, \ldots, \Delta_{1}-1\right\}$ be the collection of homomorphic images of $\Delta$ acquired by the contraction of the vertices $e_{3 l_{4}+1}^{1}: l_{4}=1,2, \ldots, \Delta_{1}-1$, with the vertex $e_{3 \Delta_{2}}^{2}$ in $\Delta$. Suppose $r$ shows itself as the remainder of $\left(l_{4} /\left(\Delta_{1}-l_{4}\right)\right)$, then graphically, we make four partitions of $\mathrm{H}_{5}$ as follows:

(i) $\Omega_{l_{4}}^{5}: \Delta_{1}-2 l_{4}>1$ (Figure 11(a))

(ii) $\Omega_{l_{4}}^{5}: \Delta_{1}-2 l_{4}=1$ (Figure 11(b))

(iii) $\Omega_{l_{4}}^{5}: \Delta_{1}-2 l_{4}<1$ and $\Delta_{1}-l_{4}>r+1$ (Figure 11(c))

(iv) $\Omega_{l_{4}}^{5}: \Delta_{1}-2 l_{4}<1$ and $\Delta_{1}-l_{4}=r+1$ (Figure 11(d))

From all the homomorphic images presented in these figures, it is not intricated to check that every one is the mirror image of itself. This lemma can be proved by using the same procedure as that for Lemma 1.

Lemma 6. If we contract the vertices $e_{3 l_{5}+1}^{2}: l_{5}=1,2, \ldots$, $\Delta_{2}-1$, with the vertex $e_{3 \Delta_{2}}^{2}$ in the circuit $\Delta$, then there arise $\Delta_{2}-1$ distinct homomorphic images of $\Delta$ and $3\left(l_{5}+2\right)$ pairs of contracted vertices create each homomorphic image. Moreover, the number of total pairs of contracted vertices to generate all $\Delta_{2}-1$ and their mirror homomorphic images of $\Delta$ is $3\left(\Delta_{2}^{2}+3 \Delta_{2}-4\right)$.

Let $H_{6}=\left\{\Omega_{l_{5}}^{6} ; l_{5}=1,2, \ldots, \Delta_{2}-1\right\}$ be the collection of homomorphic images of $\Delta$ acquired by the contraction of the vertices $e_{3 l_{5}+1}^{2}: l_{5}=1,2, \ldots, \Delta_{2}-1$, with the vertex $e_{3 \Delta_{2}}^{2}$ in $\Delta$. Graphically, we make two partitions of $\mathrm{H}_{5}$ as follows:

(i) $\Omega_{l_{5}}^{6}: l_{5}<\Delta_{2}-1$ (Figure 12(a))

(ii) $\Omega_{l_{5}}^{6}: l_{5}=\Delta_{2}-1$ (Figure 12(b))

From all the homomorphic images presented in Figures 12(a) and 12(b), it is not intricated to check that no one is the mirror image of itself. This lemma can be proved by using the same procedure as that for Lemma 1 .

Let $k_{1} \in\{3,4,5\}$.

Lemma 7. If we contract the vertices $e_{3 l_{\left(6, k_{1}\right)+1}}^{k_{1}}: l_{\left(6, k_{1}\right)}=$ $1,2, \ldots, \Delta_{k_{1}}-1$, with the vertex $e_{3 \Delta}^{2}$ in the circuit $\Delta$, then for each $k_{1}$, there arise $\Delta_{k_{1}}-1$ distinct homomorphic images of $\Delta$ and $3\left(l_{\left(6, k_{1}\right)}+2\right)$ pairs of contracted vertices create each homomorphic image. Moreover, the number of total pairs of contracted vertices to generate all $\Delta_{k_{1}}-1$ and their mirror homomorphic images of $\Delta$ is $3\left(\Delta_{k_{1}}^{2}+3 \Delta_{k_{1}}-4\right)$.

For a fix value of $k_{1}$, let $H_{7}^{k_{1}^{1}}=\left\{\Omega_{l_{\left(6, k_{1}\right.}}^{7} ; l_{\left(6, k_{1}\right)}=\right.$ $\left.1,2, \ldots, \Delta_{k_{1}}-1\right\}$ be the collection of homomorphic images of $\Delta$ acquired by the contraction of the vertices $e_{3 l_{\left(6, k_{1}\right)}+1}^{k_{1}}: l_{\left(6, k_{1}\right)}=$ $1,2, \ldots, \Delta_{k_{1}}-1$, with the vertex $e_{3 \Delta_{2}}^{2}$ in $\Delta$. Figures 13-15 present $H_{7}^{k_{1}}$ graphically. From all the homomorphic images presented in these figures, it is not intricated to check that no one is the mirror image of itself. This lemma can be proved by using the same procedure as that for Lemma 1.

Lemma 8. If we contract the vertices $e_{3 l_{7}+1}^{3}: l_{7}=1,2, \ldots$, $\Delta_{4}-1$, with the vertex $e_{3 \Delta_{3}}^{3}$ in the circuit $\Delta$, then there arise 


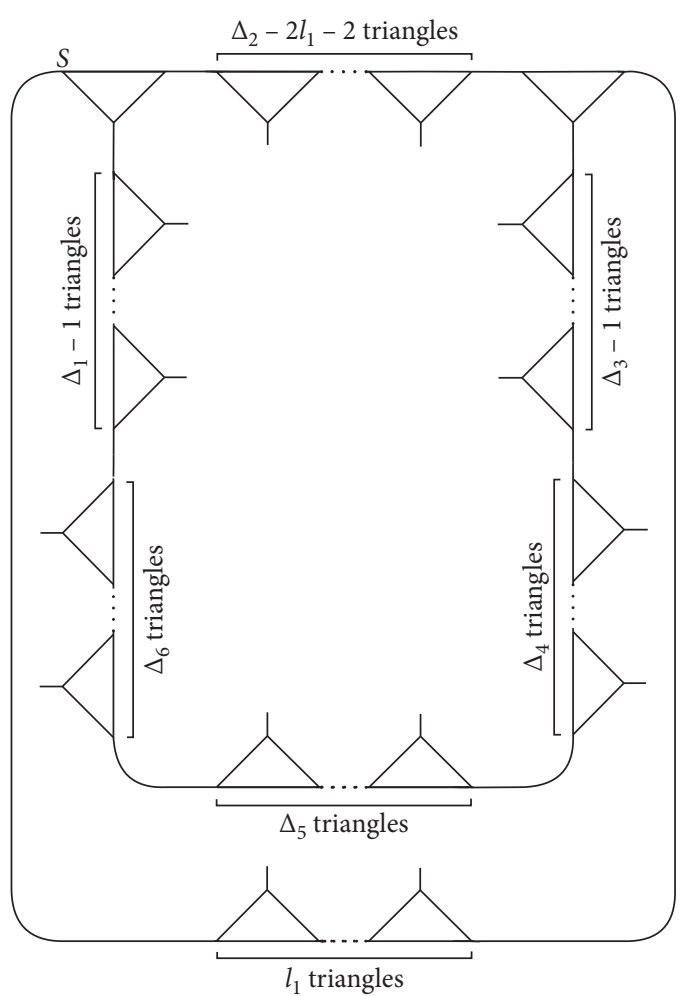

(a)

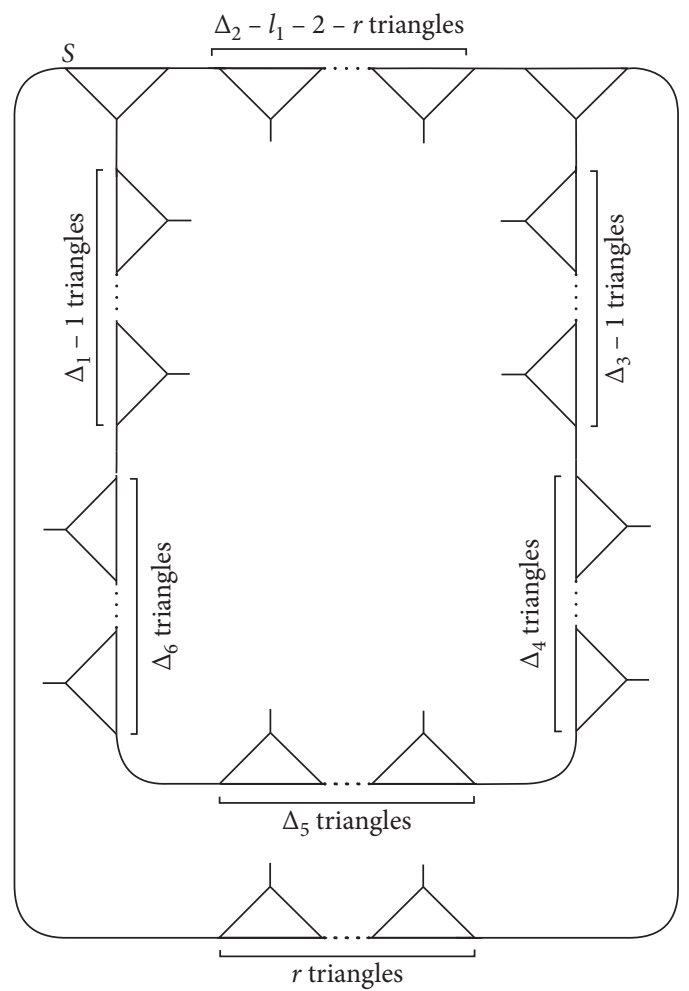

(c)

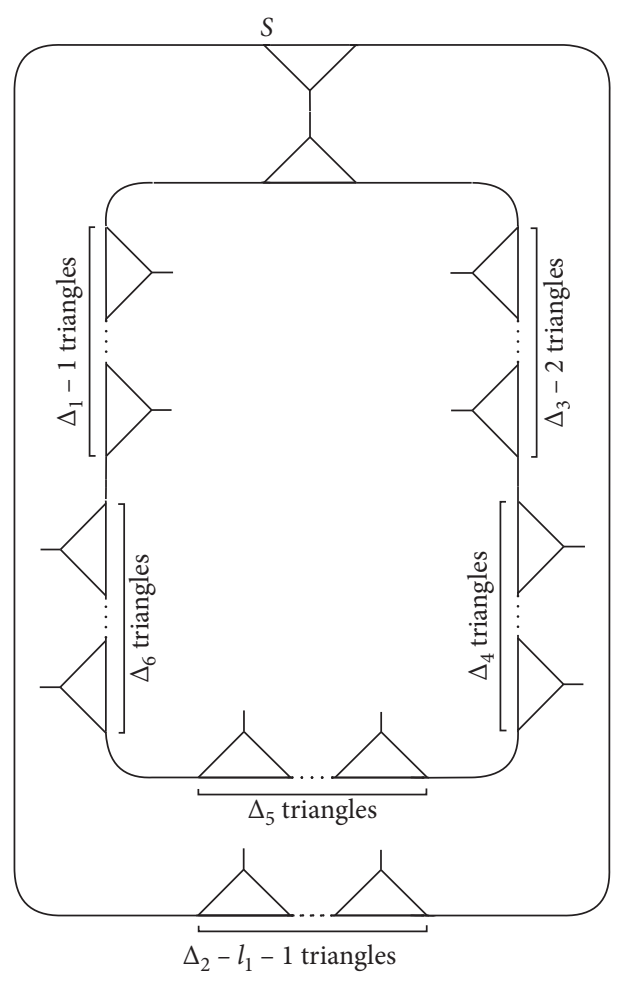

(b)

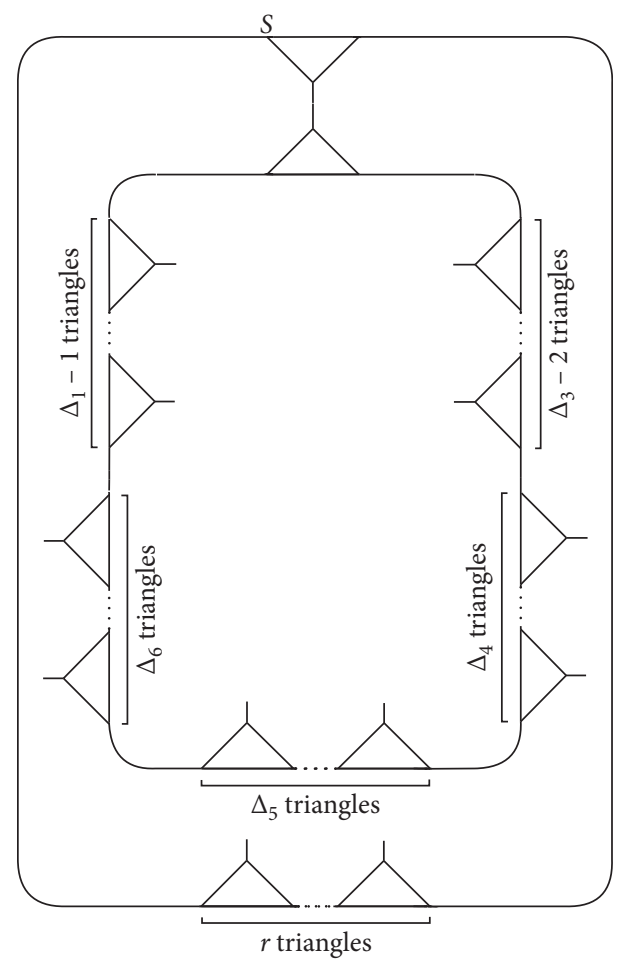

(d)

FIGURE 6: (a) Homomorphic images $\Omega_{l_{1}}^{2}: \Delta_{2}-2 l_{1}>1$. (b) Homomorphic images $\Omega_{l_{1}}^{2}: \Delta_{2}-2 l_{1}=1$. (c) Homomorphic images $\Omega_{l_{1}}^{2}: \Delta_{2}-$ $2 l_{1}<1$ and $\Delta_{2}-l_{1}>r+1$. (d) Homomorphic images $\Omega_{l_{1}}^{2}: \Delta_{2}-2 l_{1}<1$ and $\Delta_{2}-l_{1}=r+1$. 


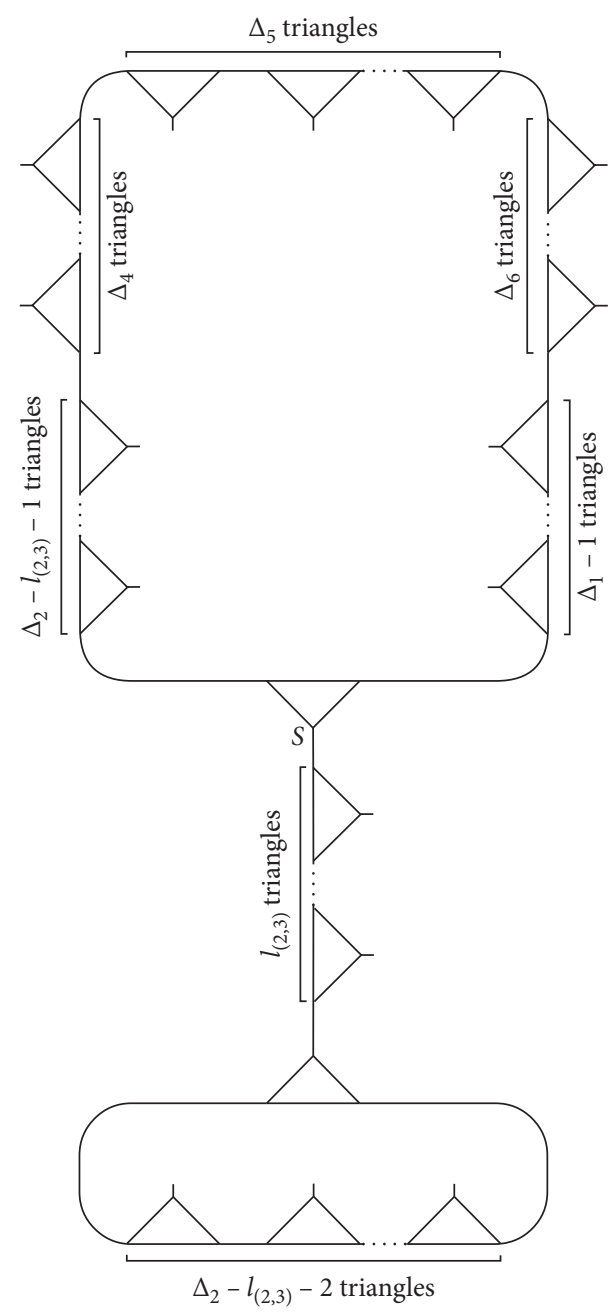

FIgURE 7: Homomorphic images $\Omega_{l_{(2,3)}^{3}}^{3}$.

$\Delta_{4}-1$ distinct homomorphic images of $\Delta$ and $3\left(l_{7}+2\right)$ pairs of contracted vertices create each homomorphic image. Moreover, the number of total pairs of contracted vertices to generate all $\Delta_{4}-1$ and their mirror homomorphic images of $\Delta$ is $3\left(\Delta_{4}^{2}+3 \Delta_{4}-4\right)$.

Let $H_{8}=\left\{\Omega_{l_{7}}^{8} ; l_{7}=1,2, \ldots, \Delta_{4}-1\right\}$ be the collection of homomorphic images of $\Delta$ acquired by the contraction of the vertices $e_{3 l_{7+1}}^{3}: l_{7}=1,2, \ldots, \Delta_{4}-1$, with the vertex $e_{3 \Delta_{3}}^{3}$ in $\Delta$. Figure 16 presents $H_{8}$ graphically. From all the homomorphic images presented in Figure 16, it is not intricated to check that no one is the mirror image of itself. This lemma can be proved by using the same procedure as that for Lemma 1.

Lemma 9. If we contract the vertices $e_{3 l_{7}+1}^{4}: l_{7}=1,2, \ldots$, $\Delta_{4}-1$, with the vertex $e_{3 \Delta_{3}}^{3}$ in the circuit $\Delta$, then there arise $\Delta_{4}-1$ distinct homomorphic images of $\Delta$ and $3\left(l_{7}+2\right)$ pairs of contracted vertices create each homomorphic image. Moreover, the number of total pairs of contracted vertices to generate all $\Delta_{1}-1$ and their mirror homomorphic images of $\Delta$ is $(3 / 2)\left(\Delta_{4}^{2}+3 \Delta_{4}-4\right)$.

Let $H_{9}=\left\{\Omega_{l_{7}}^{9} ; l_{7}=1,2, \ldots, \Delta_{4}-1\right\}$ be the collection of homomorphic images of $\Delta$ acquired by the contraction of the vertices $e_{3 l_{7}+1}^{4}: l_{7}=1,2, \ldots, \Delta_{4}-1$, with the vertex $e_{3 \Delta_{3}}^{3}$ in $\Delta$. Suppose $r$ shows itself as the remainder of $\left(l_{7} / \Delta_{4}-l_{7}\right)$, then graphically we make four partitions of $\mathrm{H}_{9}$ as follows:

(i) $\Omega_{l_{7}}^{9}: \Delta_{4}-2 l_{7}>1$ (Figure $17(a)$ )

(ii) $\Omega_{l_{7}}^{9}: \Delta_{4}-2 l_{7}=1$ (Figure 17(b))

(iii) $\Omega_{l_{7}^{9}}^{9}: \Delta_{4}-2 l_{7}<1$ and $\Delta_{4}-l_{7}>r+1$ (Figure 17(c))

(iv) $\Omega_{l_{7}}^{9}: \Delta_{4}-2 l_{7}<1$ and $\Delta_{4}-l_{7}=r+1$ (Figure $17(d)$ )

From all the homomorphic images presented in these figures, it is not intricated to check that every one is the mirror image of itself. This lemma can be proved by using the same procedure as that for Lemma 1.

Lemma 10. If we contract the vertices $e_{3 l_{8}+1}^{4}: l_{8}=0,1,2, \ldots$, $\Delta_{4}-1$, with the vertex $e_{3 \Delta_{4}}^{4}$ in the circuit $\Delta$, then there arise $\Delta_{4}$ distinct homomorphic images of $\Delta$ and $3\left(l_{8}+2\right)$ pairs of contracted vertices create each homomorphic image. Moreover, the number of total pairs of contracted vertices to generate all $\Delta_{4}$ and their mirror homomorphic images of $\Delta$ is $3\left(\Delta_{4}^{2}+3 \Delta_{4}-2\right)$.

Let $H_{10}=\left\{\Omega_{l_{8}}^{10} ; l_{8}=0,1,2, \ldots, \Delta_{4}-1\right\}$ be the collection of homomorphic images of $\Delta$ acquired by the contraction of the vertices $e_{3 l_{8}+1}^{4}: l_{8}=0,1,2, \ldots, \Delta_{4}-1$, with the vertex $e_{3 \Delta_{4}}^{4}$ in $\Delta$. Graphically, we make two partitions of $H_{10}$ as follows:

(i) $\Omega_{l_{8}}^{10}: l_{8}<\Delta_{4}-1$ (Figure 18(a))

(ii) $\Omega_{l_{8}}^{10}: l_{8}=\Delta_{4}-1$ (Figure $18(b)$ )

From all the homomorphic images presented in these figures, it is not intricated to check that no one is the mirror image of itself except $\Omega_{0}^{10}$. This lemma can be proved by using the same procedure as that for Lemma 1.

Lemma 11. If we contract the vertices $e_{3 l_{9}+1}^{5}: l_{9}=0,1,2, \ldots$, $\Delta_{5}-1$, with the vertex $e_{3 \Delta_{4}}^{4}$ in the circuit $\Delta$, then there arise $\Delta_{5}$ distinct homomorphic images of $\Delta$ and $3\left(l_{9}+2\right)$ pairs of contracted vertices create each homomorphic image. Moreover, the number of total pairs of contracted vertices to generate all $\Delta_{5}$ and their mirror homomorphic images of $\Delta$ is $(3 / 2)\left(\Delta_{5}^{2}+3 \Delta_{5}\right)$.

Let $H_{11}=\left\{\Omega_{l_{9}}^{11} ; l_{9}=0,1,2, \ldots, \Delta_{5}-1\right\}$ be the collection of homomorphic images of $\Delta$ acquired by the contraction of the vertices $e_{3 l_{9}+1}^{5}: l_{9}=0,1,2, \ldots, \Delta_{5}-1$, with the vertex $e_{3 \Delta_{4}}^{4}$ in $\Delta$. Figure 19 presents $H_{11}$ graphically. From all the homomorphic images presented in Figure 19, it is not intricated to check that every one is the mirror image of itself. This lemma can be proved by using the same procedure as that for Lemma 1.

Lemma 12. If we contract the verticese $3_{3 l_{1}+1}^{3}: l_{10}=0,1,2, \ldots$, $\Delta_{3}-1$, with the vertex $e_{3 \Delta_{4}}^{4}$ in the circuit $\Delta$, then there arise $\Delta_{3}$ distinct homomorphic images of $\Delta$ and $3\left(l_{10}+2\right)$ pairs of contracted vertices create each homomorphic image. Moreover, the number of total pairs of contracted vertices to generate all $\Delta_{3}$ and their mirror homomorphic images of $\Delta$ is $3\left(\Delta_{3}^{2}+3 \Delta_{3}\right)$.

Let $H_{12}=\left\{\Omega_{l_{10}}^{12} ; l_{10}=0,1,2, \ldots, \Delta_{3}-1\right\}$ be the collection of homomorphic images of $\Delta$ acquired by the contraction of the vertices $e_{3 l_{10}+1}^{3}: l_{10}=0,1,2, \cdots, \Delta_{3}-1$, with the vertex $e_{3 \Delta_{4}}^{4}$ 


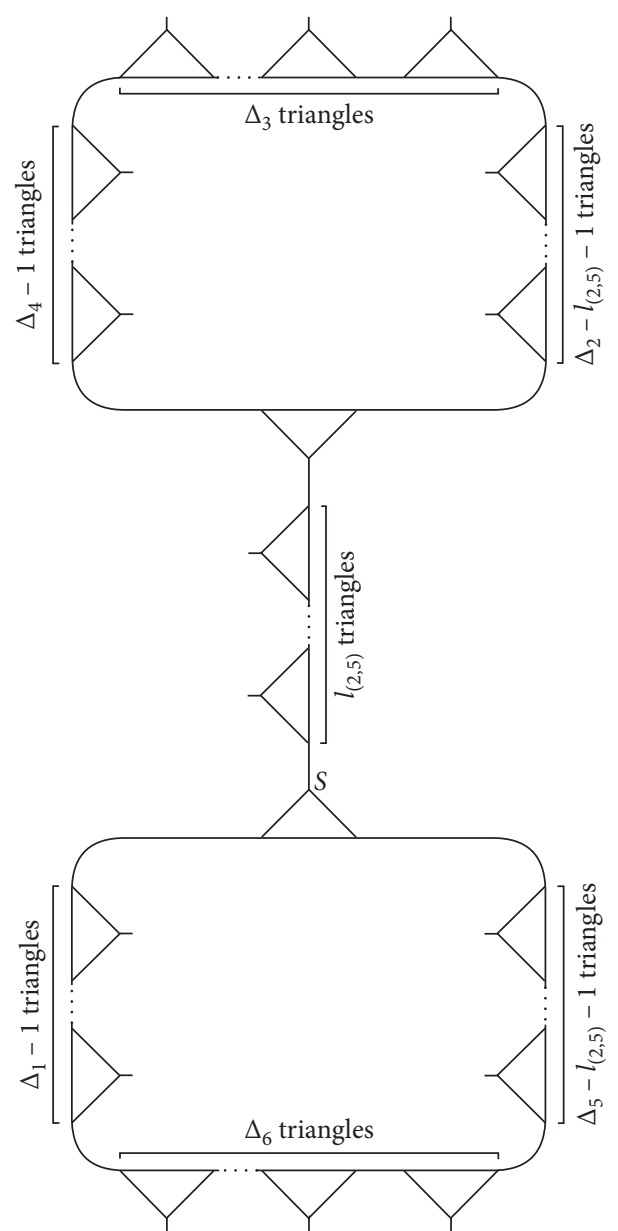

FIGURE 8: Homomorphic images $\Omega_{l_{(2,5)}^{3}}$.

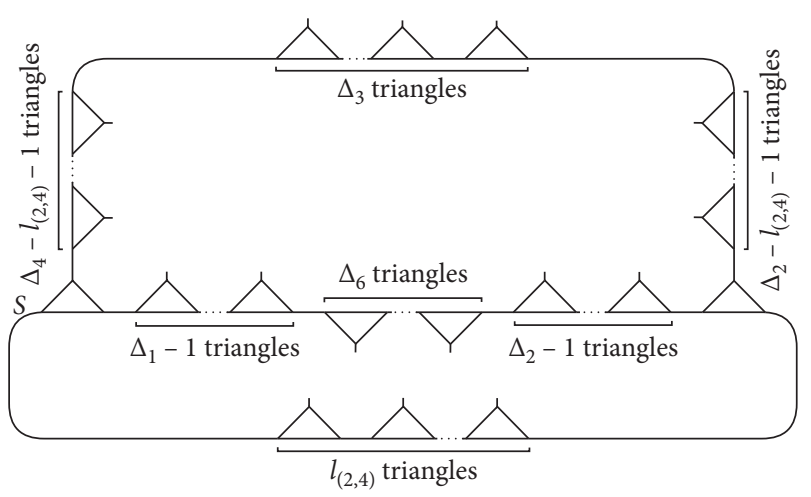

FIGURE 9: Homomorphic images $\Omega_{l_{(2,4)}^{3}}^{3}$.

in $\Delta$. Suppose $r$ shows itself as the reminder of $\left(l_{10} /\left(\Delta_{3}-l_{10}\right)\right)$, then graphically we make four partitions of $H_{12}$ as follows:

(i) $\Omega_{l_{10}}^{12}: \Delta_{3}-2 l_{10}>1$ (Figure 20(a))

(ii) $\Omega_{l_{10}}^{12}: \Delta_{3}-2 l_{10}=1$ (Figure 20(b))

(iii) $\Omega_{l_{10}}^{12}: \Delta_{3}-2 l_{10}<1$ and $\Delta_{3}-l_{10}>r+1$ (Figure 20(c))

(iv) $\Omega_{l_{10}}^{12}: \Delta_{3}-2 l_{10}<1$ and $\Delta_{3}-l_{10}=r+1$ (Figure 20(d))

From all the homomorphic images presented in these figures, it is not intricated to check that no one is the mirror image of itself. This lemma can be proved by using the same procedure as that for Lemma 1.

Lemma 13. If we contract the vertices $e_{3 l_{1}+1}^{2}: l_{1}=0,1,2, \ldots$, $\Delta_{2}-1$, with the vertex $e_{3 \Delta_{5}}^{5}$ in the circuit $\Delta$, then there arise $\Delta_{2}$ distinct homomorphic images of $\Delta$ and $3\left(l_{1}+2\right)$ pairs of contracted vertices create each homomorphic image. Moreover, the number of total pairs of contracted vertices to generate all $\Delta_{2}$ and their mirror homomorphic images of $\Delta$ is $(3 / 2)\left(\Delta_{2}^{2}+3 \Delta_{2}\right)$.

Let $H_{13}=\left\{\Omega_{l_{1}}^{13} ; l_{1}=0,1,2, \ldots, \Delta_{2}-1\right\}$ be the collection of homomorphic images of $\Delta$ acquired by the contraction of the vertices $e_{3 l_{1}+1}^{2}: l_{1}=0,1,2, \ldots, \Delta_{2}-1$, with the vertex $e_{3 \Delta_{5}}^{5}$ in $\Delta$. Figure 21 presents $H_{13}$ graphically. From all the homomorphic images presented in Figure 21, it is not intricated to check that every one is the mirror image of itself. This lemma can be proved by using the same procedure as that for Lemma 1.

Lemma 14. If we contract the vertices $e_{3 l_{11}+1}^{3}: l_{11}=0,1,2, \ldots, \Delta_{3}-1$, with the vertex $e_{3 \Delta_{5}}^{5}$ in the circuit $\Delta$, then there arise $\Delta_{3}$ distinct homomorphic images of $\Delta$ and $3\left(l_{11}+2\right)$ pairs of contracted vertices create each homomorphic image. Moreover, the number of total pairs of 


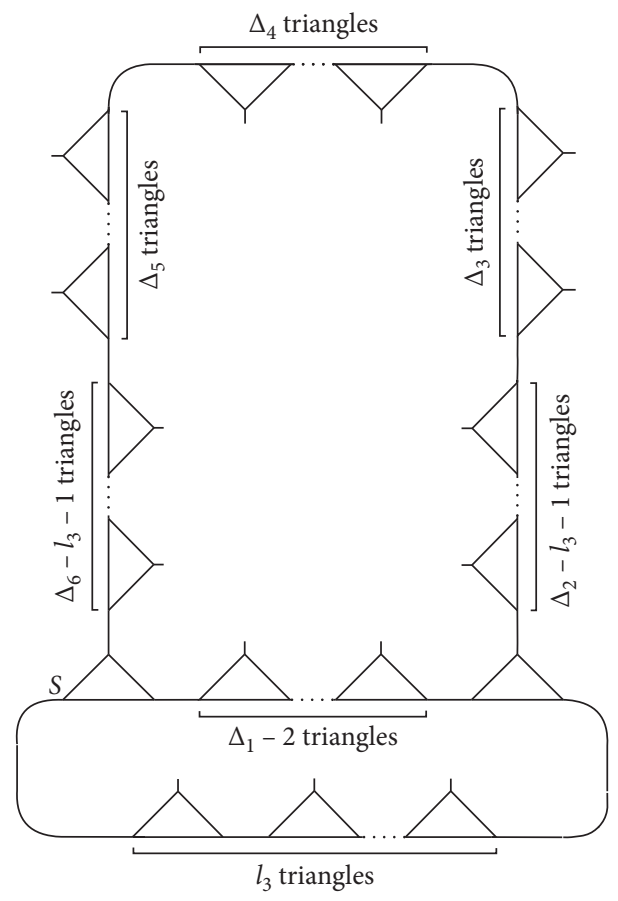

Figure 10: Homomorphic images $\Omega_{l_{3}}^{4}$.

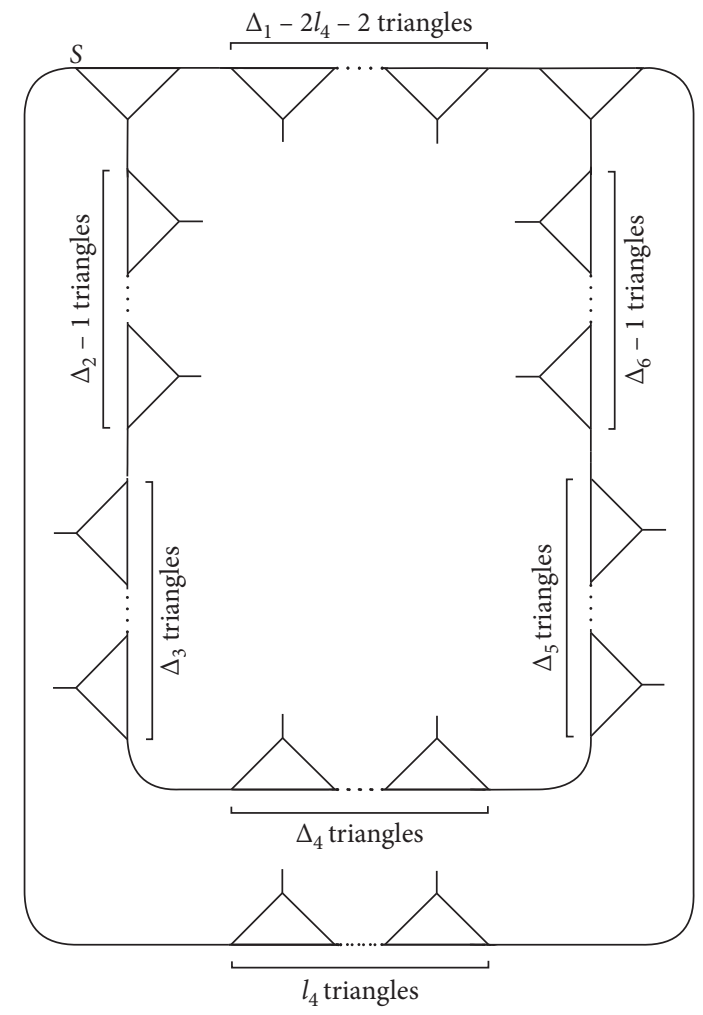

(a)

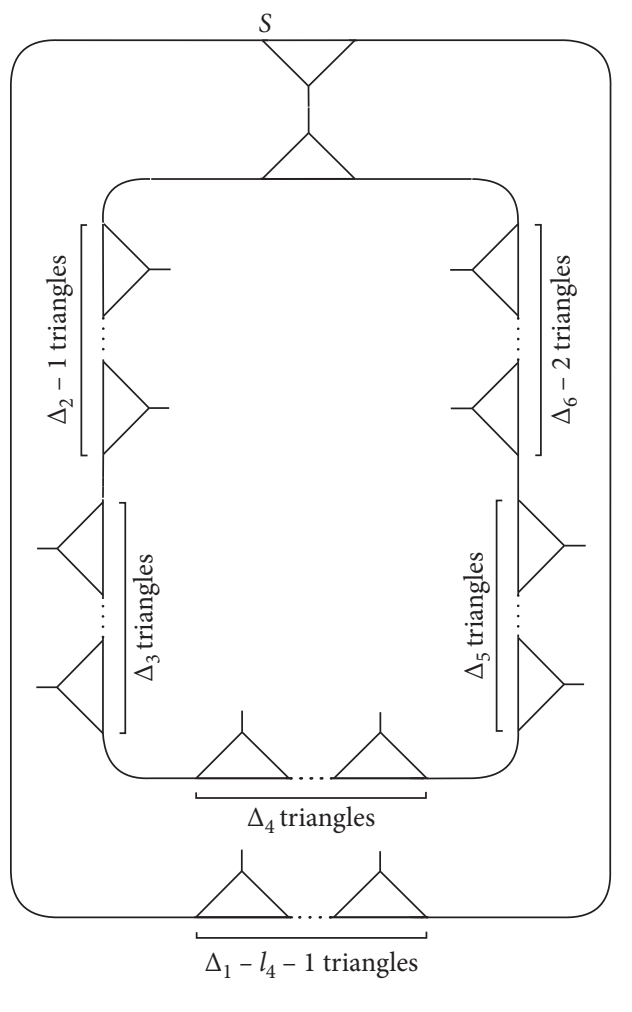

(b)

Figure 11: Continued. 


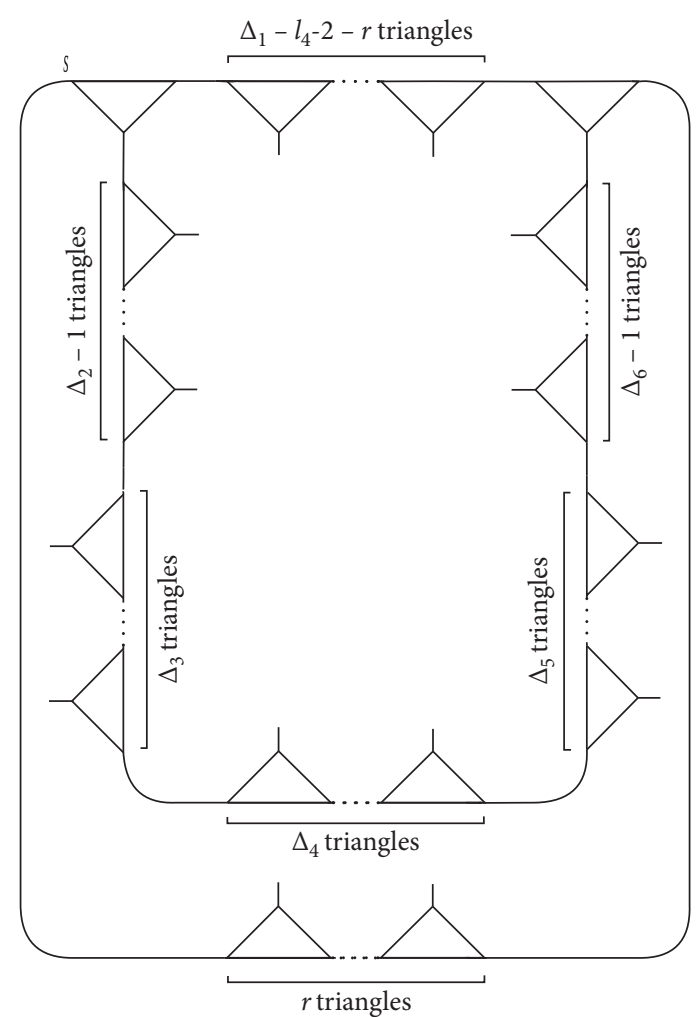

(c)

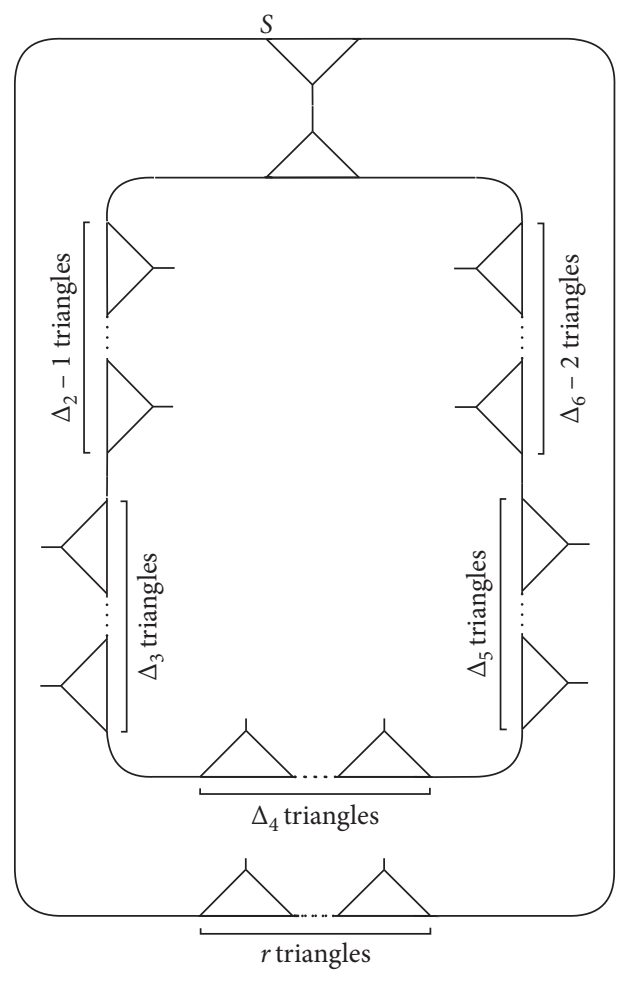

(d)

FIgURE 11: (a) Homomorphic images $\Omega_{l_{4}}^{5}: \Delta_{1}-2 l_{4}>1$. (b) Homomorphic images $\Omega_{l_{4}}^{5}: \Delta_{1}-2 l_{4}=1$. (c) Homomorphic images $\Omega_{l_{4}}^{5}: \Delta_{1}-$ $2 l_{4}<1$ and $\Delta_{1}-l_{4}>r+1$. (d) Homomorphic images $\Omega_{l_{4}}^{5}: \Delta_{1}-2 l_{4}<1$ and. $\Delta_{1}-l_{4}=\stackrel{r}{r}+1$

contracted vertices to generate all $\Delta_{3}$ and their mirror homomorphic images of $\Delta$ is $3\left(\Delta_{3}^{2}+3 \Delta_{3}-2\right)$.

Let $H_{14}=\left\{\Omega_{l_{11}}^{14} ; l_{11}=0,1,2, \ldots, \Delta_{3}-1\right\}$ be the collection of homomorphic images of $\Delta$ acquired by the contraction of the vertices $e_{3 l_{11}+1}^{3}: l_{11}=0,1,2, \ldots, \Delta_{3}-1$, with the vertex $e_{3 \Delta_{5}}^{5}$ in $\Delta$. Figure 22 presents $H_{14}$ graphically. From all the homomorphic images presented in Figure 22, it is not intricated to check that no one is the mirror image of itself except $\Omega_{0}^{14}$. This lemma can be proved by using the same procedure as that for Lemma 1.

Lemma 15. If we contract the vertices $e_{3 l_{7}+1}^{4}: l_{7}=1,2, \ldots$, $\Delta_{4}-1$, with the vertex $e_{3 \Delta_{5}}^{5}$ in the circuit $\Delta$, then there arise $\Delta_{4}-1$ distinct homomorphic images of $\Delta$ and $3\left(l_{7}+2\right)$ pairs of contracted vertices create each homomorphic image. Moreover, the number of total pairs of contracted vertices to generate all $\Delta_{4}-1$ and their mirror homomorphic images of $\Delta$ is $3\left(\Delta_{4}^{2}+3 \Delta_{4}-4\right)$.

Let $H_{15}=\left\{\Omega_{l_{7}}^{15} ; l_{7}=1,2, \ldots, \Delta_{4}-1\right\}$ be the collection of homomorphic images of $\Delta$ acquired by the contraction of the vertices $e_{3 l_{7}+1}^{4}: l_{7}=1,2, \ldots, \Delta_{4}-1$, with the vertex $e_{3 \Delta_{5}}^{5}$ in $\Delta$. Figure 23 presents $H_{15}$ graphically. From all the homomorphic images presented in Figure 23, it is not intricated to check that no one is the mirror image of itself.

This lemma can be proved by using the same procedure as that for Lemma 1.
Lemma 16. If we contract the vertices $e_{3 l_{12}+1}^{5}: l_{12}=0,1,2$, $\ldots, \Delta_{5}-1$, with the vertex $e_{3 \Delta_{5}}^{5}$ in the circuit $\Delta$, then there arise $\Delta_{5}$ distinct homomorphic images of $\Delta$ and $3\left(l_{12}+2\right)$ pairs of contracted vertices create each homomorphic image. Moreover, the number of total pairs of contracted vertices to generate all $\Delta_{5}$ and their mirror homomorphic images of $\Delta$ is $3\left(\Delta_{5}^{2}+3 \Delta_{5}\right)$.

Let $H_{16}=\left\{\Omega_{l_{12}}^{16} ; l_{12}=0,1,2, \ldots, \Delta_{5}-1\right\}$ be the collection of homomorphic images of $\Delta$ acquired by the contraction of the vertices $e_{3 l_{12}+1}^{5}: l_{12}=0,1,2, \ldots, \Delta_{5}-1$, with the vertex $e_{3 \Delta_{5}}^{5}$ in $\Delta$. Graphically, we make two partitions of $H_{16}$ as follows:

(i) $\Omega_{l_{12}}^{16}: l_{12}<\Delta_{5}-1$ (Figure 24(a))

(ii) $\Omega_{l_{12}}^{16}: l_{12}=\Delta_{5}-1$ (Figure 24(b))

From all the homomorphic images presented in these figures, it is not intricated to check that no one is the mirror image of itself. This lemma can be proved by using the same procedure as that for Lemma 1.

Lemma 17. If we contract the vertices $e_{3 l_{13}+1}^{3}: l_{13}=1,2, \ldots$, $\Delta_{3}-1$, with the vertex $e_{3 \Delta_{6}}^{6}$ in the circuit $\Delta$, then there arise $\Delta_{3}-1$ distinct homomorphic images of $\Delta$ and $3\left(l_{13}+2\right)$ pairs of contracted vertices create each homomorphic image. Moreover, the number of total pairs of contracted vertices to generate all $\Delta_{3}-1$ and their mirror homomorphic images of $\Delta$ is $(3 / 2)\left(\Delta_{3}^{2}+3 \Delta_{3}-4\right)$. 


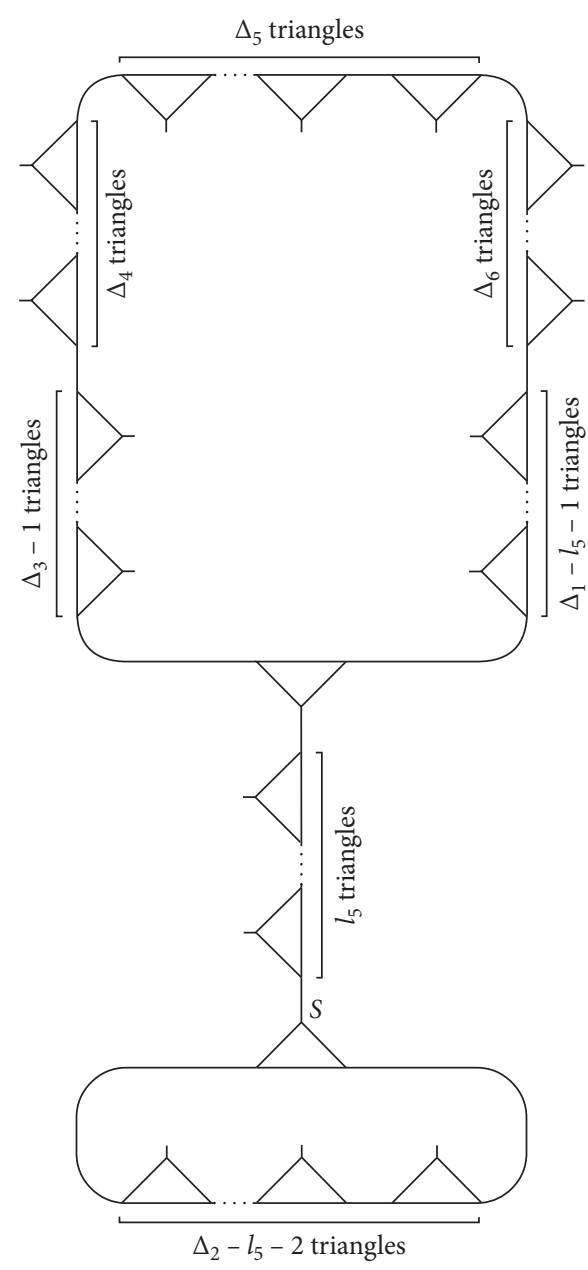

(a)

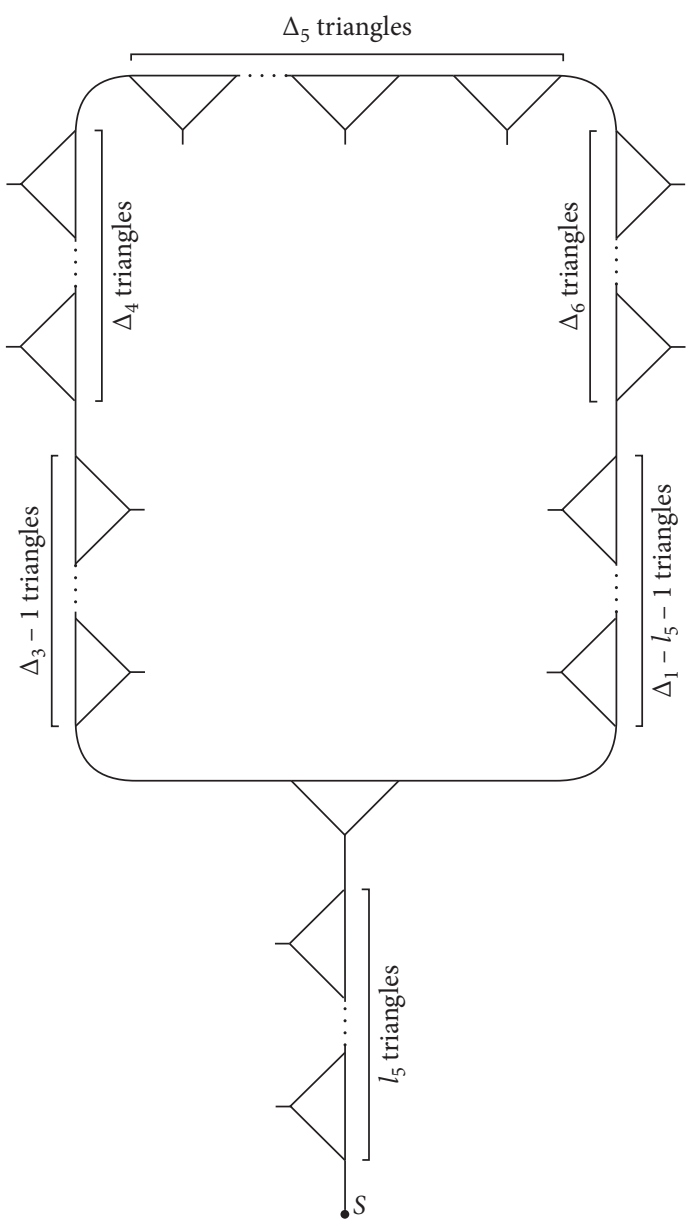

(b)

FiguRE 12: (a) Homomorphic images $\Omega_{l_{5}}^{6}: l_{5}<\Delta_{2}-1$. (b) Homomorphic images $\Omega_{l_{5}}^{6}: l_{5}=\Delta_{2}-1$.

Let $H_{17}=\left\{\Omega_{l_{13}}^{17} ; l_{13}=1,2, \ldots, \Delta_{3}-1\right\}$ be the collection of homomorphic images of $\Delta$ acquired by the contraction of the vertices $e_{3 l_{13}+1}^{3}: l_{13}=1,2, \ldots, \Delta_{3}-1$, with the vertex $e_{3 \Delta_{6}}^{6}$ in $\Delta$. Figure 25 presents $H_{17}$ graphically. From all the homomorphic images presented in Figure 25, it is not intricated to check that every one is the mirror image of itself. This lemma can be proved by using the same procedure as that for Lemma 1.

Lemma 18. If we contract the vertices $e_{3 l_{1}+1}^{1}: l_{14}=\Delta_{2}+1$, $\Delta_{2}+2, \ldots, \Delta_{1}-1$, with the vertex $e_{3 \Delta_{1}}^{1}$ in the circuit $\Delta$, then there arise $\Delta_{1}-\Delta_{2}-1$ distinct homomorphic images of $\Delta$ and $3\left(\Delta_{2}+2\right)$ pairs of contracted vertices create each homomorphic image. Moreover, the number of total pairs of contracted vertices to generate all $\Delta_{1}-\Delta_{2}-1$ and their mirror homomorphic images of $\Delta$ is $6\left(\Delta_{2}+2\right)\left(\Delta_{1}-\Delta_{2}-1\right)$.

Proof. Let $\quad H_{18}=\left\{\Omega_{l_{14}}^{18} ; l_{14}=\Delta_{2}+1, \Delta_{2}+2, \ldots, \Delta_{1}-1\right\}$ (Figure 26) be the collection of homomorphic images of $\Delta$ acquired by the contraction of the vertices $e_{3 l_{14}+1}^{1}: l_{14}=\Delta_{2}+$ $1, \Delta_{2}+2, \ldots, \Delta_{1}-1$, with the vertex $e_{3 \Delta_{1}}^{1}$ in $\Delta$, where $e_{3 l_{14}}^{1} \Delta_{1}^{1}$ and $e_{3 \Delta_{1}}^{1}$ are fixed by the words $(p q)^{1}$ $-l_{14}\left(p q^{-1}\right)^{\Delta_{6}}(p q)^{\Delta_{5}}\left(p q^{-1}\right)^{\Delta_{4}}(p q)^{\Delta_{3}}\left(p q^{-1}\right)^{\Delta_{2}}(p q)^{l_{14}}$ and $(p q)^{\Delta_{2}}\left(p q^{-1}\right)^{\Delta_{3}}(p q)^{\Delta_{4}}\left(p q^{-1}\right)^{\Delta_{5}}(p q)^{\Delta_{2}}\left(p q^{-1}\right)^{\Delta_{3}}(p q)^{\Delta_{4}}$ $\left(p q^{-1}\right)^{\Delta_{5}}(p q)^{\Delta_{6}}\left(p q^{-1}\right)^{\Delta_{1}}$. It is easy to verify that $P_{1}=$ $q^{-1}\left(p q^{-1}\right)^{\Delta_{1}-l_{14}-1}$ and $P_{2}=(p q)^{\Delta_{2}}\left(p q^{-1}\right)^{\Delta_{3}}(p q)^{\Delta_{4}}\left(p q^{-1}\right)^{\Delta_{5}}$ $(p q)^{\Delta_{6}}\left(p q^{-1}\right)^{l_{14}} p$ are the possible paths between $e_{3 l_{14}+1}^{1}$ and $e_{3 \Delta_{1}}^{1}$ (Figure 4). This implies that, for each $l_{14}$, the homomorphic image $\Omega_{l_{14}}^{18}$ has a vertex $s$ fixed by $P_{1}$ and $P_{2}$. Thus,

$$
\begin{aligned}
E_{18}= & \left\{e, q, q^{-1}, p, p q, p q^{-1}, p q p,(p q)^{2}, p q p q^{-1}, \ldots,\right. \\
& \left.(p q)^{\Delta_{2}} p,,(p q)^{\Delta_{2}+1},(p q)^{\Delta_{2}} p q^{-1}\right\}
\end{aligned}
$$

is the family of elements in $\operatorname{PSL}(2, \mathbb{Z})$ such that $\forall x \in E_{18}, \Delta$ contains the vertices $\left(e_{3 l_{14}+1}^{1}\right) x$ and $\left(e_{3 \Delta_{1}}^{1}\right) x$. This gives that the cardinality of $E_{18}$, that is, $3\left(\Delta_{2}+2\right)$ is the number of contracted pairs of vertices to produce the homomorphic image $\Omega_{l_{14}}^{18}$ (Theorem 2).

Now, we will prove that all the homomorphic images in $H_{18}$ are distinct and no one is the mirror image of itself.

Let $\Omega_{m}^{18}$ and $\Omega_{n}^{18}$ be any two elements of $H_{18}$, then $\Omega_{m}^{18}$ is produced by contraction of $e_{3 m+1}^{1}$ and $e_{3 \Delta_{1}}^{1}$ and $\Omega_{n}^{18}$ is produced by contraction of $e_{3 n+1}^{1}$ and $e_{3 \Delta}^{1}$. Suppose $\Omega_{m}^{18^{n}}$ and $\Omega_{n}^{18}$ are the same. This concludes that $\Omega_{m}^{18}$ is procurable also 


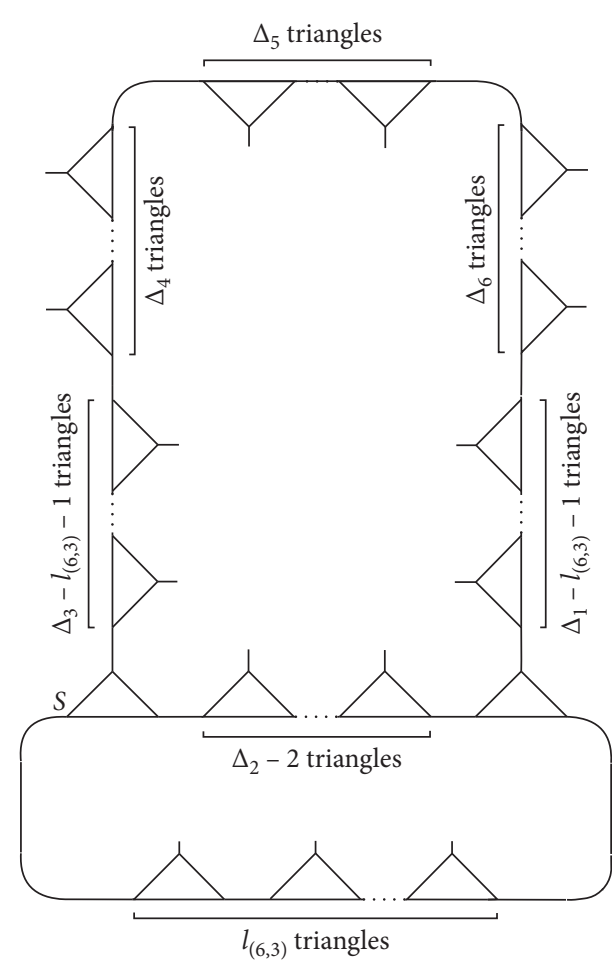

FIGURE 13: Homomorphic images $\Omega_{l_{(6,3)}^{7}}^{7}$.

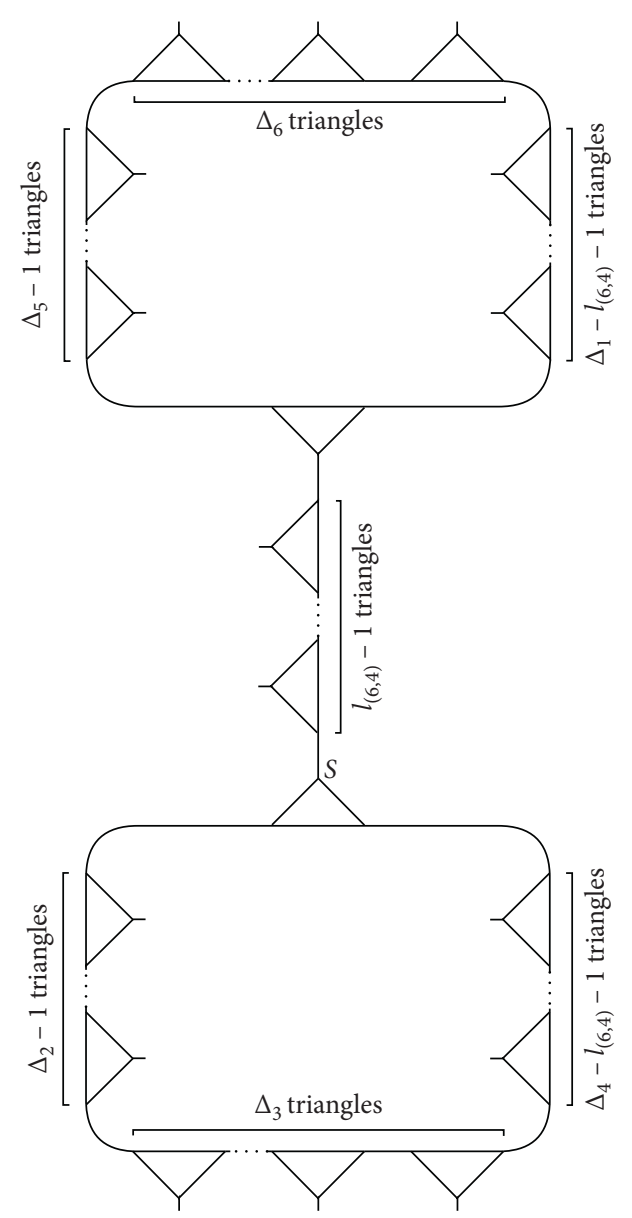

FIGURE 14: Homomorphic images $\Omega_{l_{(6,4)}^{7}}^{7}$.

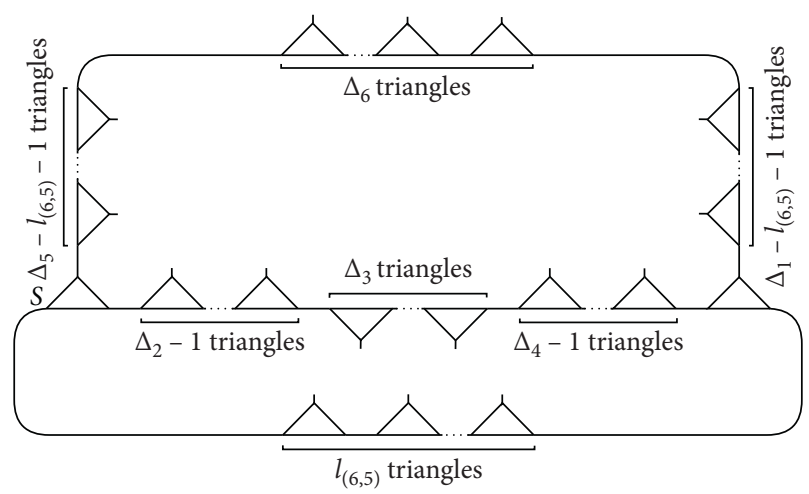

Figure 15: Homomorphic images $\Omega_{l_{(6,5)}^{7}}$.

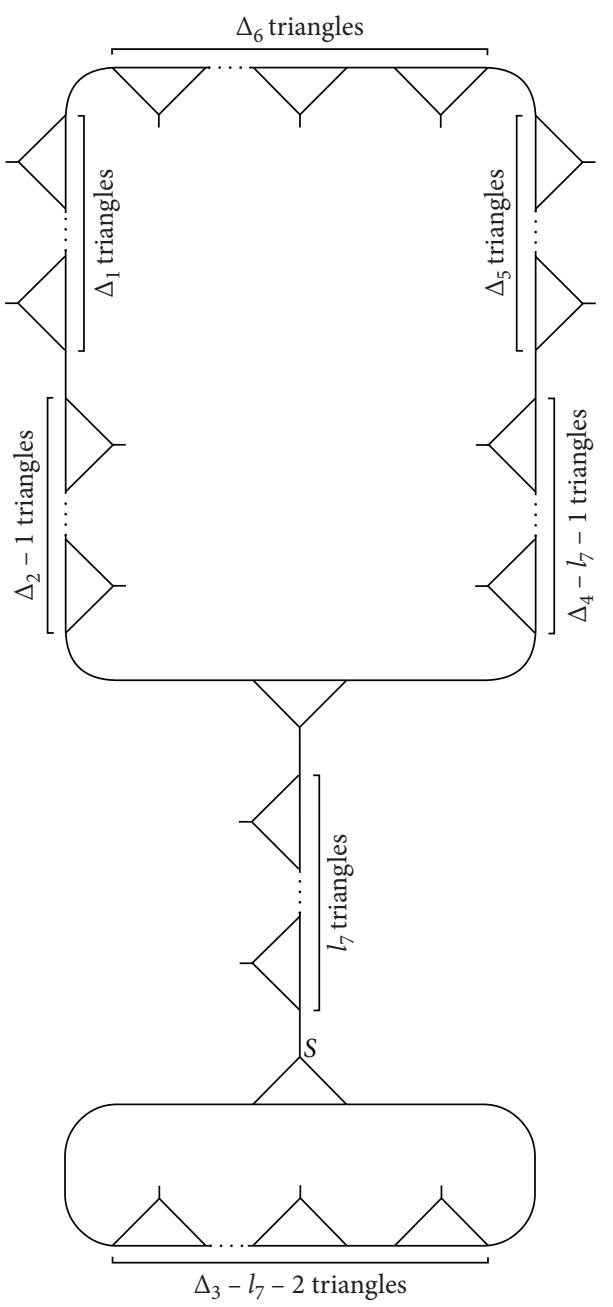

FIGURE 16: Homomorphic images $\Omega_{l_{7}}^{8}$.

by contracting $e_{3 n+1}^{1}$ and $e_{3 \Delta_{1}}^{1}$, implying that $e_{3 n+1}^{1} \leftrightarrow e_{3 \Delta_{1}}^{1}$ is one of the pairs of contracted vertices for $\Omega_{m}^{18}$. Then there must exist an element $w \in E_{18}$ such that $\left(e_{3 m+1}^{1}\right) w=e_{3 n+1}^{1}$ and $\left(e_{3 \Delta_{1}}^{1}\right) w=e_{3 \Delta_{1}}^{1} . e \in E_{18}$ is the only element such that $\left(e_{3 \Delta_{1}}^{1}\right) e=e_{3 \Delta_{1}}^{1}$ but $\left(e_{3 m+1}^{1}\right) e \neq e_{3 n+1}^{1}$. Thus, $\Omega_{m}^{18}$ and $\Omega_{n}^{18}$ are not the same, that is, by contracting $e_{3 m+1}^{1}$ and $e_{3 \Delta}^{1}$ to produce $\Omega_{m}^{18}, e_{3 n+1}^{1}$ and $e_{3 \Delta_{1}}^{1}$ are not contracted. Now, if $\Omega_{m}^{18}$ and $\Omega_{n}^{18 *}$ 


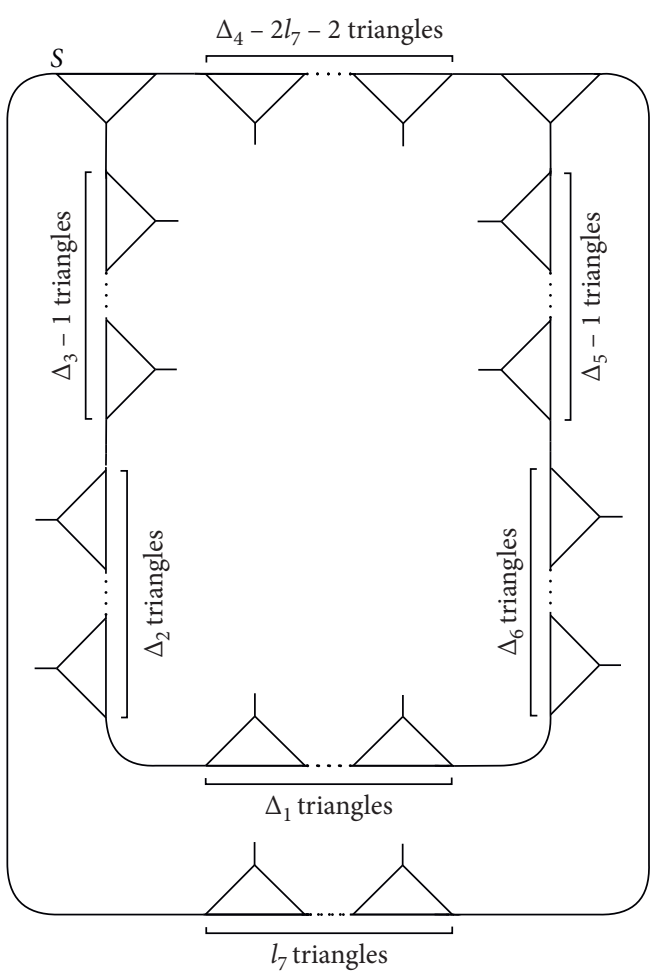

(a)

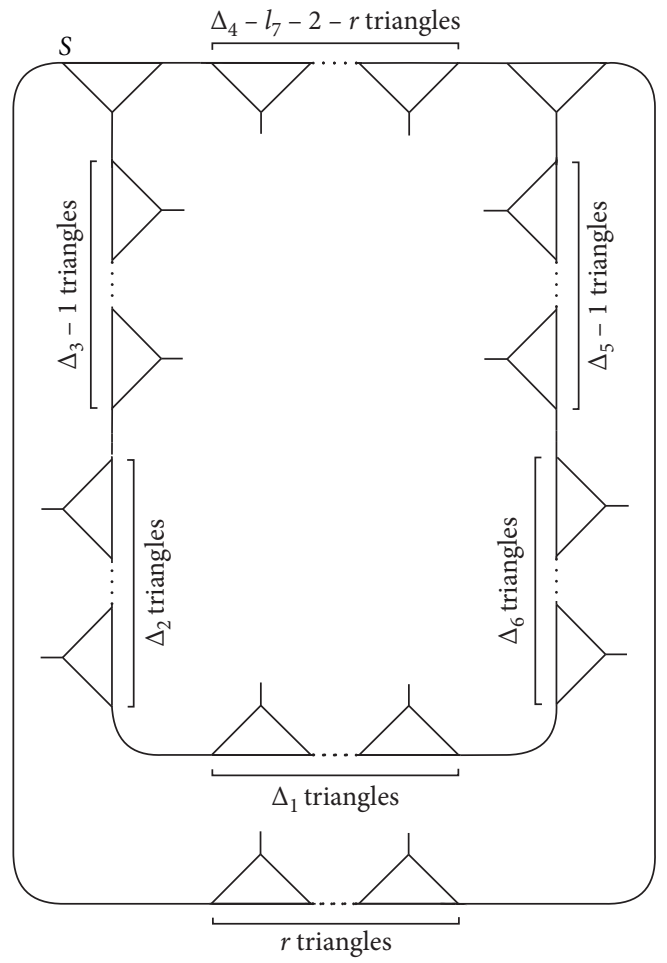

(c)

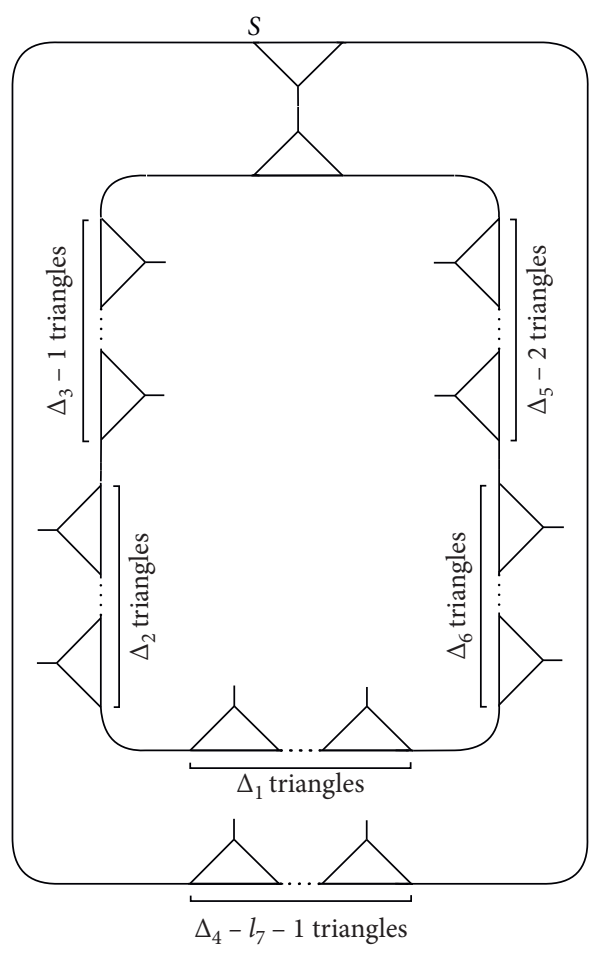

(b)

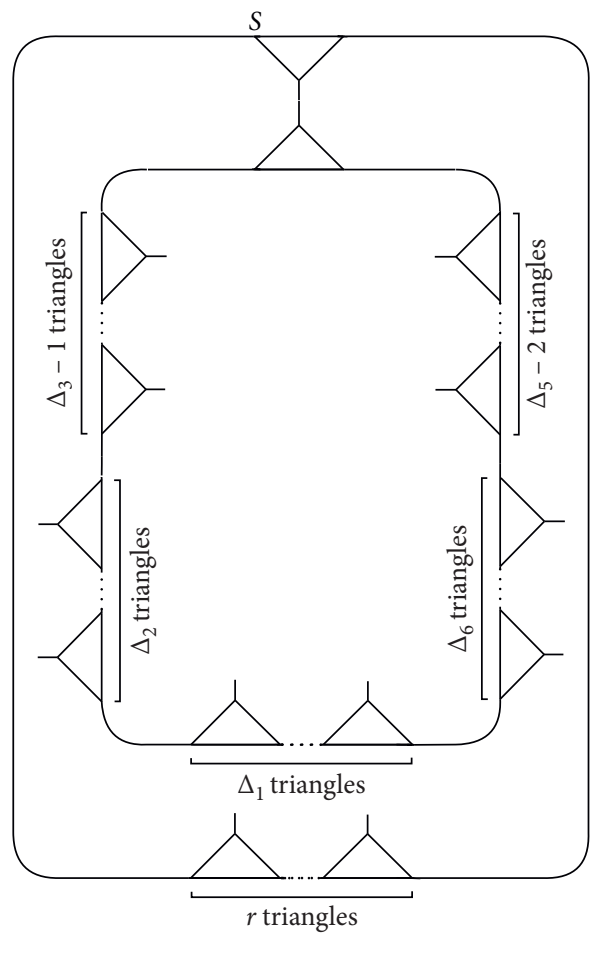

(d)

FIGURE 17: (a) Homomorphic images $\Omega_{l_{7}}^{9}: \Delta_{4}-2 l_{7}>1$. (b) Homomorphic images $\Omega_{l_{7}}^{9}: \Delta_{4}-2 l_{7}=1$. (c) Homomorphic images $\Omega_{l_{7}}^{9}: \Delta_{4}-$ $2 l_{7}<1$ and $\Delta_{4}-l_{7}>r+1$. (d) Homomorphic images $\Omega_{l_{7}}^{9}: \Delta_{4}-2 l_{7}<1$ and $\Delta_{4}-l_{7}=r+1$. 


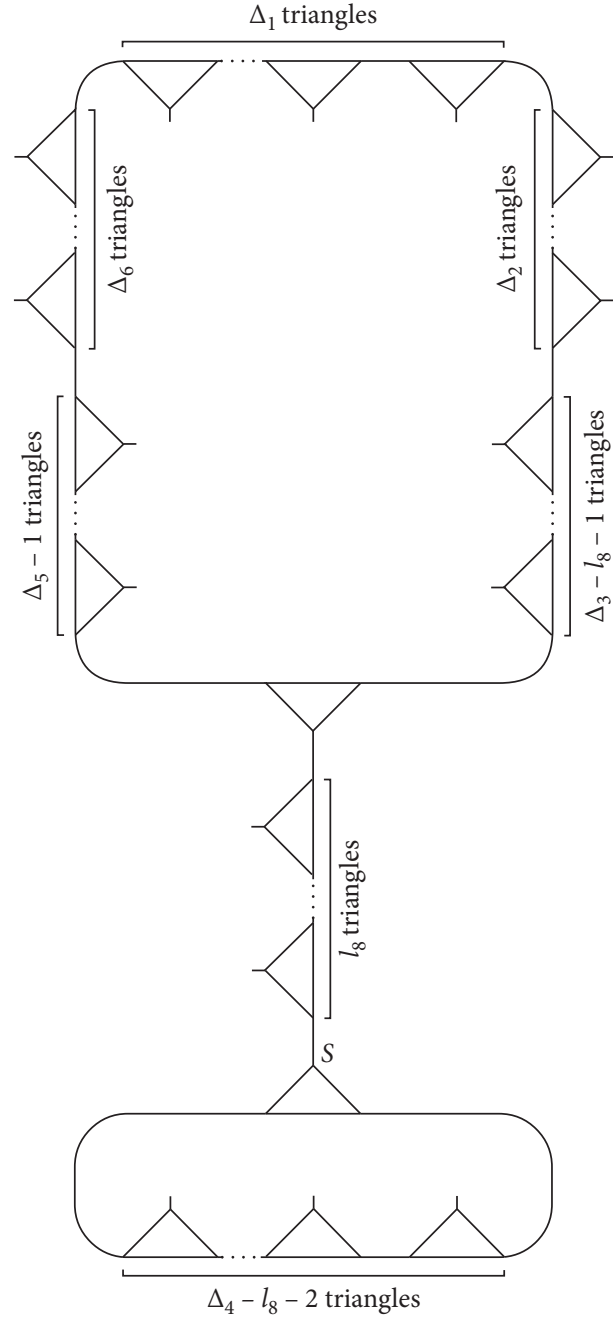

(a)

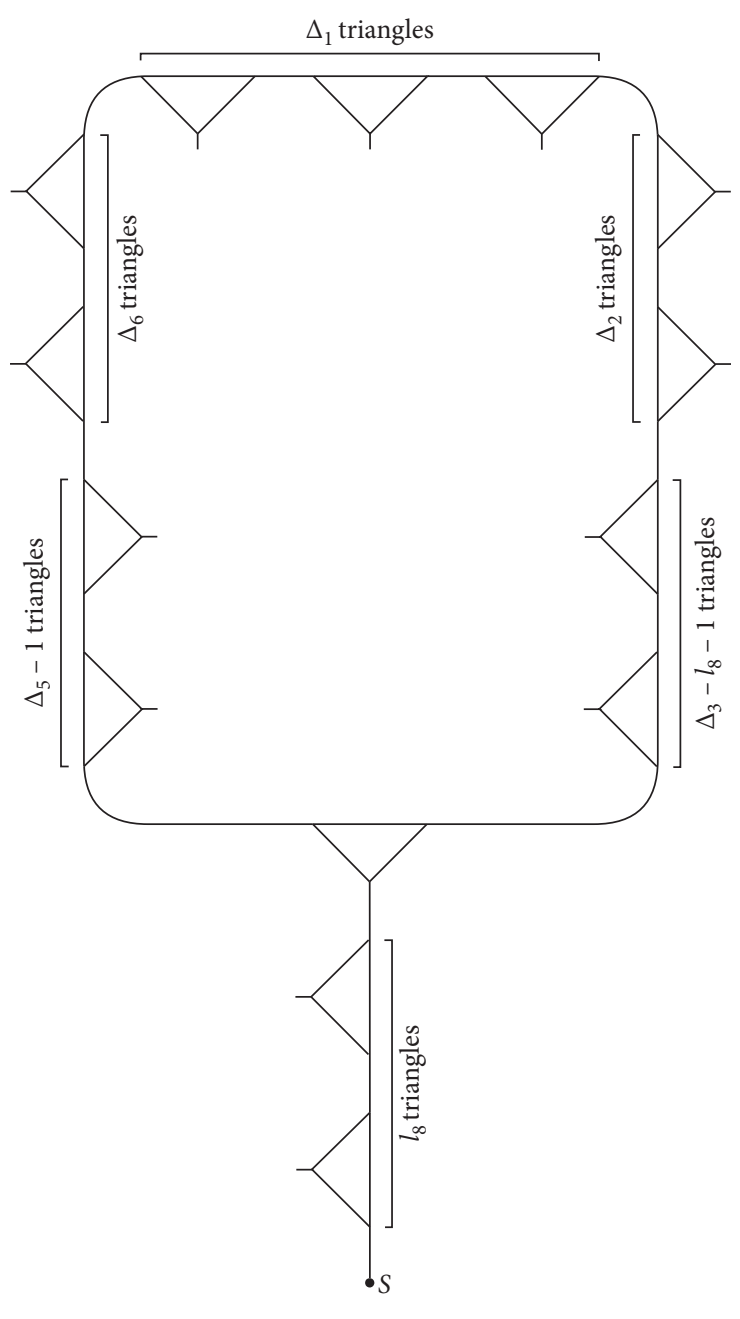

(b)

FIGURE 18: (a) Homomorphic images $\Omega_{l_{8}}^{10}: l_{8}<\Delta_{4}-1$. (b) Homomorphic images $\Omega_{l_{8}}^{10}: l_{8}=\Delta_{4}-1$.

are the same, then there must exist an element $w \in E_{18}$ such that $\left(e_{3 m+1}^{1}\right) w=e_{3 n+1}^{1 *}$ and $\left(e_{3 \Delta_{1}}^{1}\right) w=e_{3 \Delta_{1}}^{1 *}$. But there does not such an element exist in $E_{18}$. Thus, $\Omega_{m}^{18}$ is not the same as the mirror image of $\Omega_{n}^{18}$, that is, by contracting $e_{3 m+1}^{1}$ and $e_{3 \Delta}^{1}$ to create $\Omega_{m}^{18}, e_{3 n+1}^{1 *}$ and $e_{3 \Delta_{1}}^{1 *}$ are not contracted. This implies that all the elements in $H_{18}$ are distinct. Thus, $H_{18}=\left\{\Omega_{l_{14}}^{18} ; l_{14}=\Delta_{2}+1, \Delta_{2}+2, \ldots, \Delta_{1}-1\right\} \quad$ gives $\left|H_{18}\right|=\Delta_{1}-\Delta_{2}-1$.

To check does any element of $H_{18}$ is the mirror image of itself, we suppose $\Omega_{m}^{18}$ is the same to its mirror image $\Omega_{m}^{18 *}$. Then by definition, there must exist an element $w \in E_{18}$ such that $\left(e_{3 m+1}^{1}\right) w=e_{3 m+1}^{1 *}$ and $\left(e_{3 \Delta_{1}}^{1}\right) w=e_{3 \Delta_{1}}^{1 *}$. But there does not such an element exist in $E_{18}$. Thus, $\Omega_{m}^{18}$ is not the same as its mirror image, that is, by contracting $e_{3 m+1}^{1}$ and $e_{3 \Delta_{1}}^{1}$ to create $\Omega_{m}^{18}, e_{3 m+1}^{1 *}$ and $e_{3 \Delta_{1}}^{1 *}$ are not contracted. Hence, there are

$$
2 \times\left|E_{18}\right| \times\left|H_{18}\right|=6\left(\Delta_{2}+2\right)\left(\Delta_{1}-\Delta_{2}-1\right)
$$

pairs of contracting vertices to produce all the homomorphic images in $H_{18}$

\footnotetext{
Let $\eta_{1}=\left\{\begin{array}{ll}0, & \text { if } \Delta_{1}+\Delta_{4}=0(\bmod 2) \\ 1, & \text { otherwise. }\end{array}\right.$.
}

Lemma 19. If we contract the vertices $e_{3 l_{15}+1}^{1}: l_{15}=\Delta_{4}+1, \Delta_{4}+2, \ldots,\left(\left(\Delta_{1}+\Delta_{4}-\eta_{1}\right) / 2\right)$, with the vertex $e_{3 \Delta_{3}}^{3}$ in the circuit $\Delta$, then there arise $\left(\left(\Delta_{1}-\Delta_{4}-\eta_{1}\right) / 2\right)$ distinct homomorphic images of $\Delta$ and $3\left(\Delta_{4}+2\right)$ pairs of contracted vertices create each homomorphic image. Moreover, the number of total pairs of contracted vertices to generate all $\left(\left(\Delta_{1}-\Delta_{4}-\eta_{1}\right) / 2\right)$ and their mirror homomorphic images of $\Delta$ is $3\left(\Delta_{4}+2\right)\left(\Delta_{1}-\Delta_{4}-1\right)$.

Proof. Let

$H_{19}=\left\{\Omega_{l_{1}}^{19} ; l_{15}=\Delta_{4}+1, \Delta_{4}+2, \ldots,\left(\left(\Delta_{1}+\Delta_{4}-\eta_{1}\right) / 2\right)\right\}$

(Figure 27) be the collection of homomorphic images of $\Delta$ acquired by the contraction of the vertices $e_{3 l_{15}+1}^{1}: l_{15}=\Delta_{4}+1, \Delta_{4}+2, \ldots,\left(\left(\Delta_{1}+\Delta_{4}-\eta_{1}\right) / 2\right)$, with the vertex $e_{3 \Delta_{3}}^{3}$ in $\Delta$, where $e_{31_{15}+1}^{1}$ and $e_{3 \Delta_{3}}^{3}$ are fixed by the words $(p q)^{\Delta_{1}-l_{15}^{\Delta_{3}}}\left(p q^{-1}\right)^{\Delta_{6}}(p q)^{\Delta_{5}}\left(p q^{-1}\right)^{\Delta_{4}}(p q)^{\Delta_{3}}\left(p q^{-1}\right)^{\Delta_{2}}(p q)^{l_{15}}$ and $(p q)^{\Delta_{4}}\left(p q^{-1}\right)^{\Delta_{5}}(p q)^{\Delta_{6}}\left(p q^{-1}\right)^{\Delta_{1}}(p q)^{\Delta_{2}}\left(p q^{-1}\right)^{\Delta_{3}}$. It is easy to verify that $P_{1}=q^{-1}\left(p q^{-1}\right)^{\Delta_{1}-l_{15}-1}(p q)^{\Delta_{2}}\left(p q^{-1}\right)^{\Delta_{3}}$ and $P_{2}=(p q)^{\Delta_{4}}\left(p q^{-1}\right)^{\Delta_{5}}(p q)^{\Delta_{6}}\left(p q^{-1}\right)^{l_{15}} p$ are the possible paths between $e_{3 l_{15}+1}^{1}$ and $e_{3 \Delta_{3}}^{3}$ (Figure 4). This implies that, 


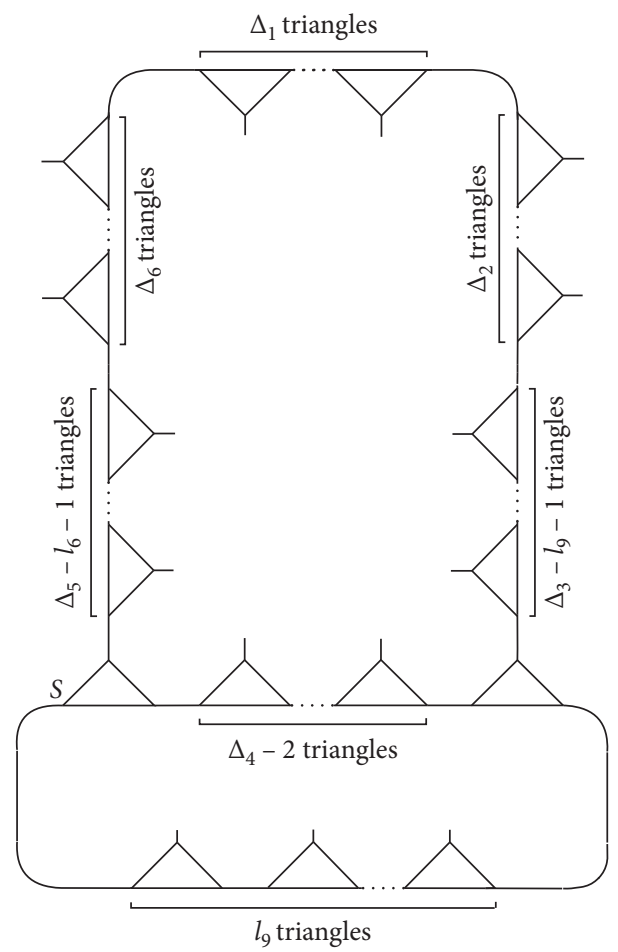

Figure 19: Homomorphic images $\Omega_{l_{9}}^{11}$.

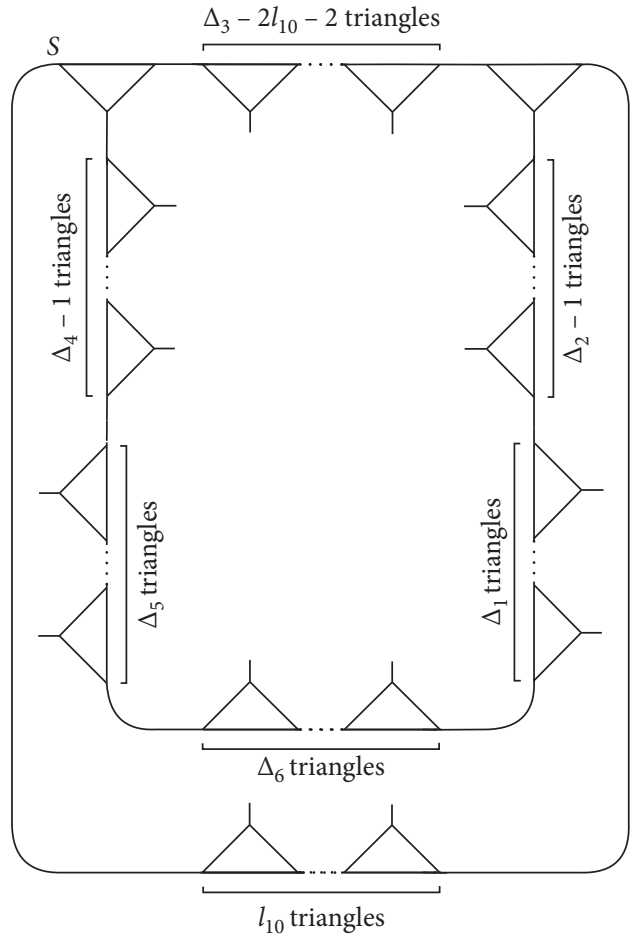

(a)

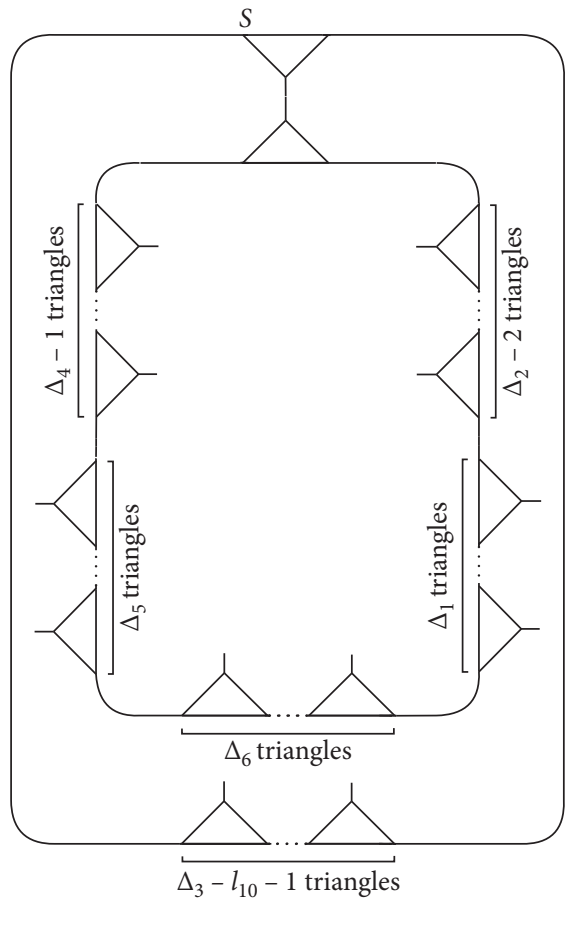

(b)

Figure 20: Continued. 


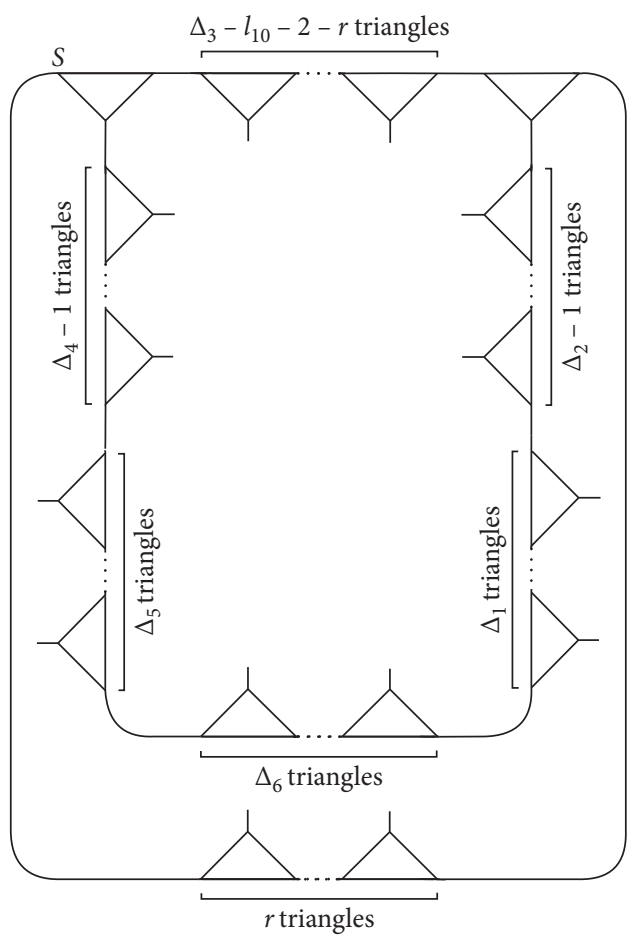

(c)

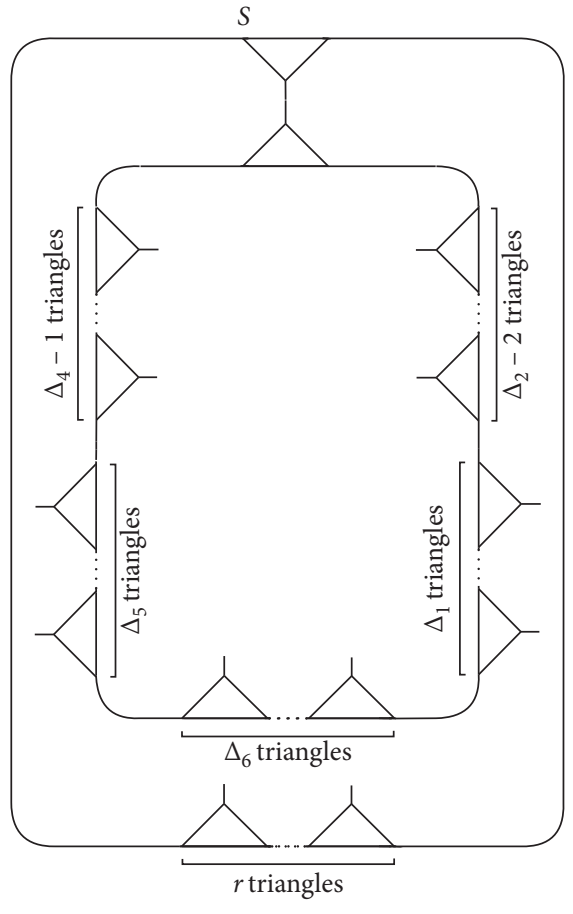

(d)

FIgURE 20: (a) Homomorphic images $\Omega_{l_{10}}^{12}: \Delta_{3}-2 l_{10}>1$. (b) Homomorphic images $\Omega_{l_{10}}^{12}: \Delta_{3}-2 l_{10}=1$. (c) Homomorphic images $\Omega_{l_{10}}^{12}: \Delta_{3}-$ $2 l_{10}<1$ and $\Delta_{3}-l_{10}>r+1$. (d) Homomorphic images $\Omega_{l_{10}}^{12}: \Delta_{3}-2 l_{10}<1$ and $\Delta_{3}-l_{10}^{10}=r+1$.

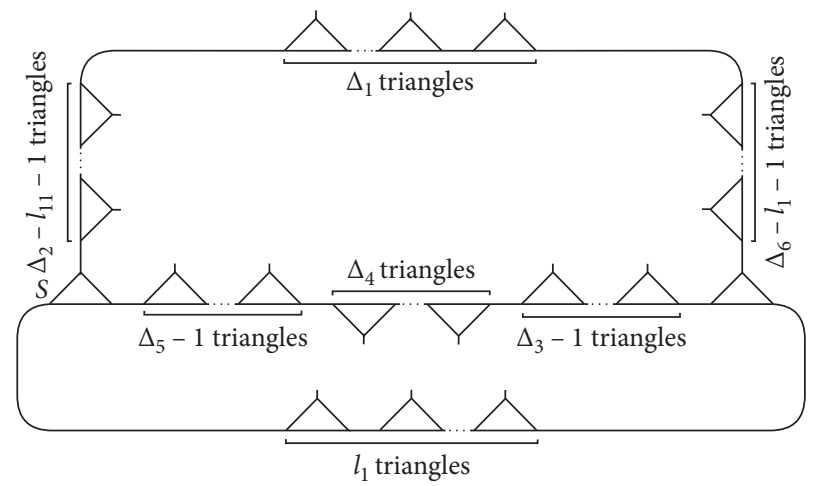

FIGURE 21: Homomorphic images $\Omega_{l_{1}}^{13}$.

for each $l_{15}$, the homomorphic image $\Omega_{l_{15}}^{19}$ has a vertex $s$ fixed by $P_{1}$ and $P_{2}$. Thus,

$$
\begin{aligned}
E_{19}= & \left\{e, q, q^{-1}, p, p q, p q^{-1}, p q p,(p q)^{2}, p q p q^{-1}, \ldots,\right. \\
& \left.(p q)^{\Delta_{4}} p,(p q)^{\Delta_{4}+1},(p q)^{\Delta_{4}} p q^{-1}\right\}
\end{aligned}
$$

is the family of elements in PSL $(2, \mathbb{Z})$ such that $\forall x \in E_{19}, \Delta$ contains the vertices $\left(e_{3 l_{15}+1}^{1}\right) x$ and $\left(e_{3 \Delta_{3}}^{3}\right) x$. This gives that the cardinality of $E_{19}$, that is, $3\left(\Delta_{4}+2\right)$ is the number of contracted pairs of vertices to produce the homomorphic image $\Omega_{l_{15}}^{17}$ (Theorem 2).

Now, we will prove that (i) for $\Delta_{1}+\Delta_{4}=1(\bmod 2)$, all the homomorphic images in $H_{19}$ are distinct and no one is the mirror image of itself, and (ii) for $\Delta_{1}+\Delta_{4}=0(\bmod 2)$, all the homomorphic images in $H_{19}$ are distinct and only $\Omega_{\left(\left(\Delta_{1}+\Delta_{4}\right) / 2\right)}^{19}$ is the mirror image of itself.

Let $\Omega_{m}^{19}$ and $\Omega_{n}^{19}$ be any two elements of $H_{19}$, then $\Omega_{m}^{19}$ is produced by contraction of $e_{3 m+1}^{1}$ and $e_{3 \Delta_{3}}^{3}$ and $\Omega_{n}^{19}$ is produced by contraction of $e_{3 n+1}^{1}$ and $e_{3 \Delta_{3}}^{3}$.

Suppose $\Omega_{m}^{19}$ and $\Omega_{n}^{19}$ are the same. This concludes that $\Omega_{m}^{19}$ is procurable also by contracting $e_{3 n+1}^{1}$ and $e_{3 \Delta_{3}}^{3}$, implying that $e_{3 n+1}^{1} \leftrightarrow e_{3 \Delta_{3}}^{3}$ is one of the pairs of contracted vertices for $\Omega_{m}^{19}$. Then there must exist an element $w \in E_{19}$ such that $\left(e_{3 m+1}^{1}\right) w=e_{3 n+1}^{1}$ and $\left(e_{3 \Delta_{3}}^{3}\right) w=e_{3 \Delta_{3}}^{3} \cdot e \in E_{19}$ is the only element such that $\left(e_{3 \Delta_{3}}^{3}\right) e=e_{3 \Delta_{3}}^{3}$ but $\left(e_{3 m+1}^{1}\right) e \neq e_{3 n+1}^{1}$. Thus, $\Omega_{m}^{19}$ and $\Omega_{n}^{19}$ are not the same, that is, by contracting $e_{3 m+1}^{1}$ and $e_{3 \Delta_{3}}^{3}$ to produce $\Omega_{m}^{19}, e_{3 n+1}^{1}$ and $e_{3 \Delta_{3}}^{3}$ are not contracted. 


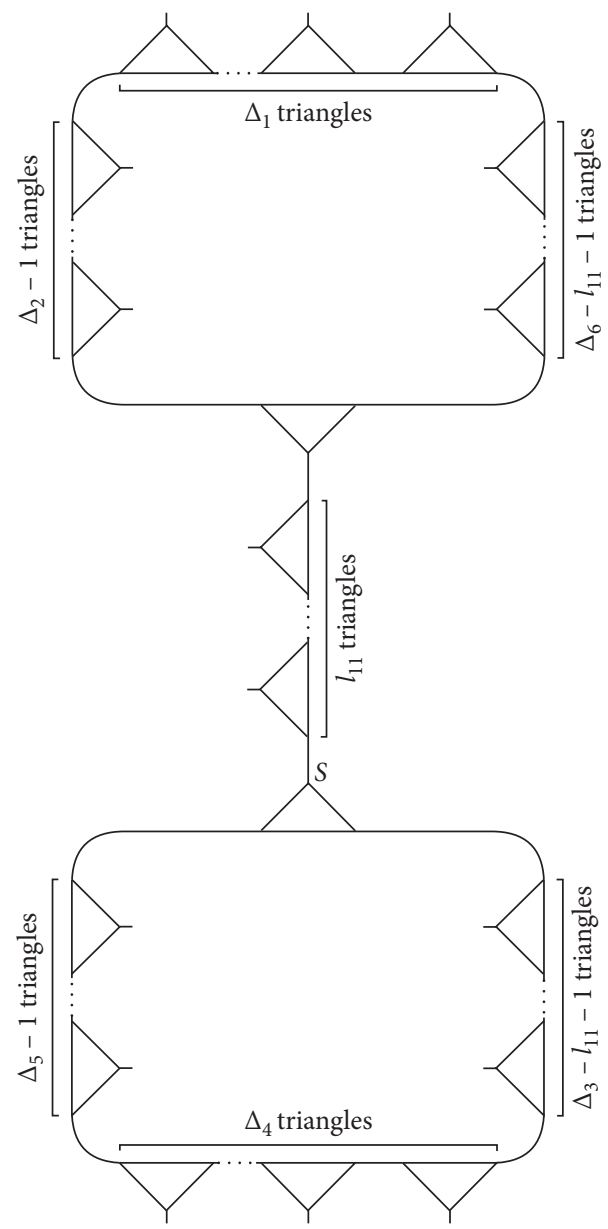

FIGURE 22: Homomorphic images $\Omega_{l_{11}}^{14}$.

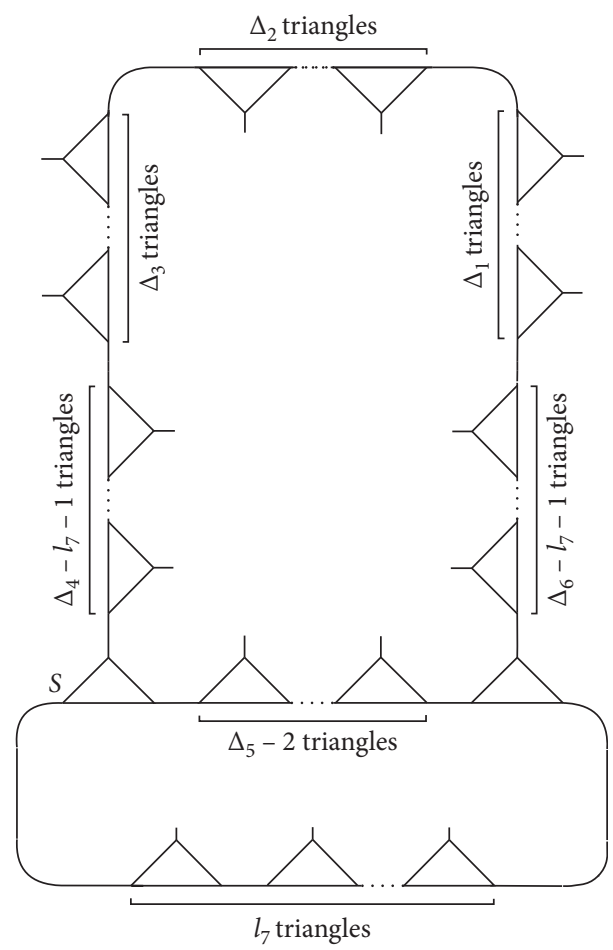

FIgURE 23: Homomorphic images $\Omega_{l_{7}}^{15}$.
Now, if $\Omega_{m}^{19}$ and $\Omega_{n}^{19 *}$ are the same, then there must exist an element $\quad w \in E_{19} \quad$ such that $\left(e_{3 m+1}^{1}\right) w=e_{3 n+1}^{1 *} \quad$ and $\left(e_{3 \Delta_{3}}^{3}\right) w=e_{3 \Delta_{3}}^{3 *} .(p q)^{\Delta_{4}} p \in E_{19}$ is the only element such that $\left(e_{3 \Delta_{3}}^{3}\right)(p q)^{\Delta_{4}} p=e_{3 \Delta_{3}}^{3 *}$ and $\left(e_{3 m+1}^{1}\right)(p q)^{\Delta_{4}} p=e_{3\left(\Delta_{1}+\Delta_{4}-m\right)+1}^{1 *}$. This implies that $\Omega_{m}^{19}$ and $\Omega_{n}^{19}$ are the mirror images of each other if and only if $n=\Delta_{1}+\Delta_{4}-m$ and $\Omega_{m}^{19}$ is the mirror image of itself if and only if $m=\Delta_{1}+\Delta_{4}-m$, that is, $m=\left(\left(\Delta_{1}+\Delta_{4}\right) / 2\right)$. Now,

(i) If $\Delta_{1}+\Delta_{4}=1(\bmod 2), \quad$ then $\forall m \in l_{15}$, $\Delta_{1}+\Delta_{4}-m>\left(\left(\Delta_{1}+\Delta_{4}-1\right) / 2\right)$, implying that $\Omega_{\Delta_{1}+\Delta_{4}-m}^{19} \notin H_{19}$. This states that no homomorphic image in $H_{19}$ is the mirror image of other. Hence, all the homomorphic images in $H_{19}$ are distinct. Thus, $H_{19}=\left\{\Omega_{l_{15}}^{19} ; l_{15}=\Delta_{4}+1, \Delta_{4}+2, \ldots,\left(\left(\Delta_{1}+\Delta_{4}-1\right) /\right.\right.$ 2)\} gives $\left|H_{19}\right|=\left(\left(\Delta_{1}-\Delta_{4}-1\right) / 2\right)$.

Also, $\left(\left(\Delta_{1}+\Delta_{4}\right) / 2\right)>\left(\left(\Delta_{1}+\Delta_{4}-1\right) / 2\right)$ gives that no homomorphic image in $H_{19}$ is the mirror image of itself. Hence, there are

$$
\begin{aligned}
2 & \times\left|E_{19}\right| \times\left|H_{19}\right|=6\left(\Delta_{4}+2\right)\left(\frac{\Delta_{1}-\Delta_{4}-1}{2}\right) \\
& =3\left(\Delta_{4}+2\right)\left(\Delta_{3}-\Delta_{4}-1\right)
\end{aligned}
$$

pairs of contracting vertices to produce all the homomorphic images in $H_{19}$.

(ii) If $\Delta_{1}+\Delta_{4}=0(\bmod 2)$, then $\forall m \in\left(l_{15} /\left\{\left(\Delta_{1}+\Delta_{4}\right)\right.\right.$ /2\}), $\Delta_{1}+\Delta_{4}-m>\left(\left(\Delta_{1}+\Delta_{4}\right) / 2\right)$, implying that $\Omega_{\Delta_{1}+\Delta_{4}-m}^{19} \notin H_{19}$. This states that no homomorphic image in $H_{19}$ is the mirror image of other. Hence, all the homomorphic images in $H_{19}$ are distinct. Thus, $H_{19}=\left\{\Omega_{l_{15}}^{19} ; l_{15}=\Delta_{4}+1, \Delta_{4}+2, \ldots,\left(\left(\Delta_{1}+\Delta_{4}\right) / 2\right)\right\}$ gives $\left|H_{19}\right|=\left(\left(\Delta_{1}-\Delta_{4}\right) / 2\right)$.

For $m=\left(\left(\Delta_{1}+\Delta_{4}\right) / 2\right)$, we have $\Delta_{1}+\Delta_{4}-m=\left(\left(\Delta_{1}+\right.\right.$ $\left.\left.\Delta_{4}\right) / 2\right) \in l_{15}$ gives $\Omega_{\left(\Delta_{1}+\Delta_{4}\right) / 2}^{19} \in H_{19}$ is the mirror image of itself. Hence, there are

$$
\begin{aligned}
& 2 \times\left|E_{19}\right| \times\left(\left|H_{19}\right|-1\right)+\left|E_{19}\right|=6\left(\Delta_{4}+2\right)\left(\frac{\Delta_{1}-\Delta_{4}}{2}-1\right) \\
& +3\left(\Delta_{4}+2\right)=3\left(\Delta_{4}+2\right)\left(\Delta_{3}-\Delta_{4}-1\right)
\end{aligned}
$$

pairs of contracting vertices to produce all the homomorphic images in $H_{19}$.

Let $k_{2} \in\{2,3\}$.

Lemma 20. If we contract the vertices $e_{3 l_{\left(3, k_{2}\right)}+1}^{k_{2}}: l_{\left(3, k_{2}\right)}=$ $\Delta_{4}+1, \Delta_{4}+2, \ldots, \Delta_{k_{2}}-1$, with the vertex $e_{3 \Delta_{3}}^{3}$ in the circuit $\Delta$, then for each $k_{2}$, there arise $\Delta_{k_{2}}-\Delta_{4}-1$ distinct homomorphic images of $\Delta$ and $3\left(\Delta_{4}+2\right)$ pairs of contracted vertices create each homomorphic image. Moreover, the number of total pairs of contracted vertices to generate all $\Delta_{k_{2}}-\Delta_{4}-1$ and their mirror homomorphic images of $\Delta$ is $6\left(\Delta_{4}+2\right)\left(\Delta_{k_{2}}-\Delta_{4}-1\right)$.

For a fix value of $k_{2}$, let

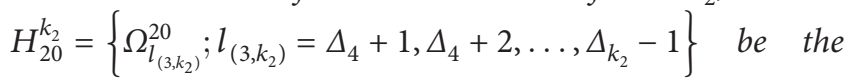
collection of homomorphic images of $\Delta$ acquired by the contraction of the vertices $e_{3 l_{\left(3, k_{2}\right)}}$ 


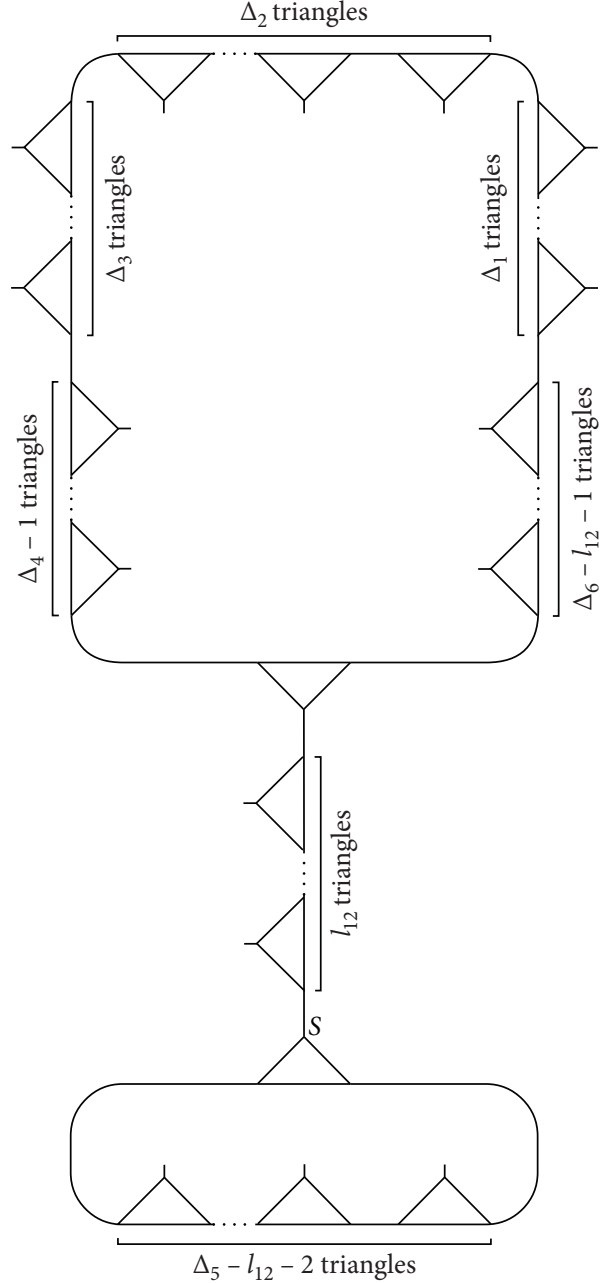

(a)

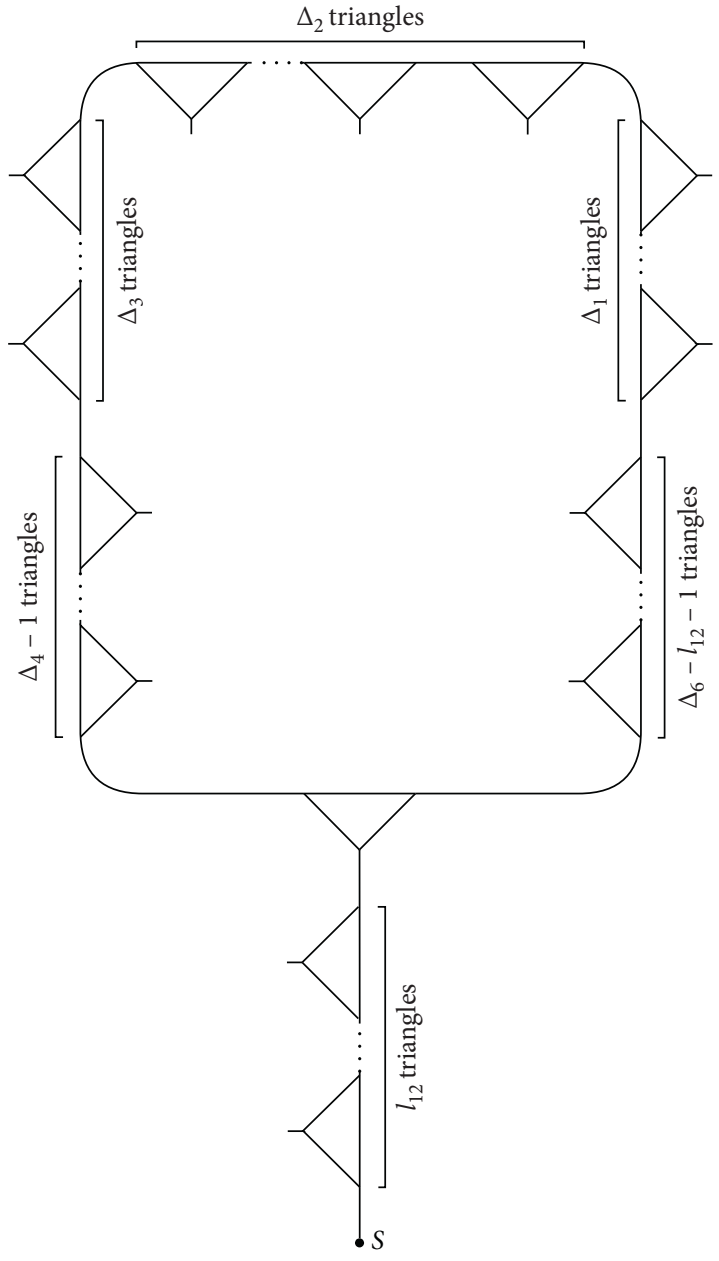

(b)

FIGURE 24: (a) Homomorphic images $\Omega_{l_{12}}^{16}: l_{12}<\Delta_{5}-1$. (b) Homomorphic images $\Omega_{l_{12}}^{16}: l_{12}=\Delta_{5}-1$.

$+1^{k_{2}}: l_{\left(3, k_{2}\right)}=\Delta_{4}+1, \Delta_{4}+2, \ldots, \Delta_{k_{2}}-1$, with the vertex $e_{3 \Delta_{3}}^{3}$ in $\Delta$. Figures 28 and 29 present $H_{3}^{k_{2}}$ graphically. From all the homomorphic images presented in these figures, it is not intricated to check that no one is the mirror image of itself.

This lemma can be proved by using the same procedure as that for Lemma 18.

Lemma 21. If we contract the vertices $e_{3 l_{16}+1}^{2}: l_{16}=\Delta_{3}+$ $1, \Delta_{3}+2, \ldots, \Delta_{2}-1$, with the vertex $e_{3 \Delta_{4}}^{4}$ in the circuit $\Delta$, then there arise $\Delta_{2}-\Delta_{3}-1$ distinct homomorphic images of $\Delta$ and $3\left(\Delta_{3}+2\right)$ pairs of contracted vertices create each homomorphic image. Moreover, the number of total pairs of contracted vertices to generate all $\Delta_{2}-\Delta_{3}-1$ and their mirror homomorphic images of $\Delta$ is $6\left(\Delta_{3}+2\right)$ $\left(\Delta_{2}-\Delta_{3}-1\right)$.

Let $H_{21}=\left\{\Omega_{l_{16}}^{21} ; l_{16}=\Delta_{3}+1, \Delta_{3}+2, \ldots, \Delta_{2}-1\right\}$ be the collection of homomorphic images of $\Delta$ acquired by the contraction of the vertices $e_{3 l_{16}+1}^{2}$ : $l_{16}=\Delta_{3}+1, \Delta_{3}+2, \ldots, \Delta_{2}-1$, with the vertex $e_{3 \Delta_{4}}^{4}$ in $\Delta$. Figure 30 presents $H_{21}$ graphically. From all the homomorphic images presented in Figure 30, it is not intricated to check that no one is the mirror image of itself. This lemma can be proved by using the same procedure as that for Lemma 18.

Let $k_{3} \in\{1,2\}$.

Lemma 22. If we contract the vertices $e_{3 l_{\left(4, k_{3}\right)}^{k_{3}+1}}: l_{\left(4, k_{3}\right)}=\Delta_{5}+1, \Delta_{5}+2, \ldots, \Delta_{k_{3}}-1$, with the vertex $e_{3 \Delta_{6}}^{6}$ in the circuit $\Delta$, then for each $k_{3}$, there arise $\Delta_{k_{3}}-\Delta_{5}-1$ distinct homomorphic images of $\Delta$ and $3\left(\Delta_{5}+2\right)$ pairs of contracted vertices create each homomorphic image. Moreover, the number of total pairs of contracted vertices to generate all $\Delta_{k_{3}}-\Delta_{5}-1$ and their mirror homomorphic images of $\Delta$ is $6\left(\Delta_{5}+2\right)\left(\Delta_{k_{3}}-\Delta_{5}-1\right)$.

For a fix value of $k_{3}$, let $H_{22}^{k_{3}}=\left\{\Omega_{l_{\left(4, k_{3}\right)}^{22}}^{2} ; l_{\left(4, k_{3}\right)}=\Delta_{5}+1, \Delta_{5}+2, \ldots, \Delta_{k_{3}}-1\right\}$ be the collection of homomorphic images of $\Delta$ acquired by the contraction of the vertices $e_{3 l_{\left(4, k_{3}\right)}+1}^{k_{3}}: l_{\left(4, k_{3}\right)}=\Delta_{5}+1$, $\Delta_{5}+2, \ldots, \Delta_{k_{3}}-1$, with the vertex $e_{3 \Delta_{6}}^{6}$ in $\Delta$. Figures 31 and 32 present $H_{22}^{k_{3}}$ graphically. From all the homomorphic images presented in these figures, it is not intricated to check that no 


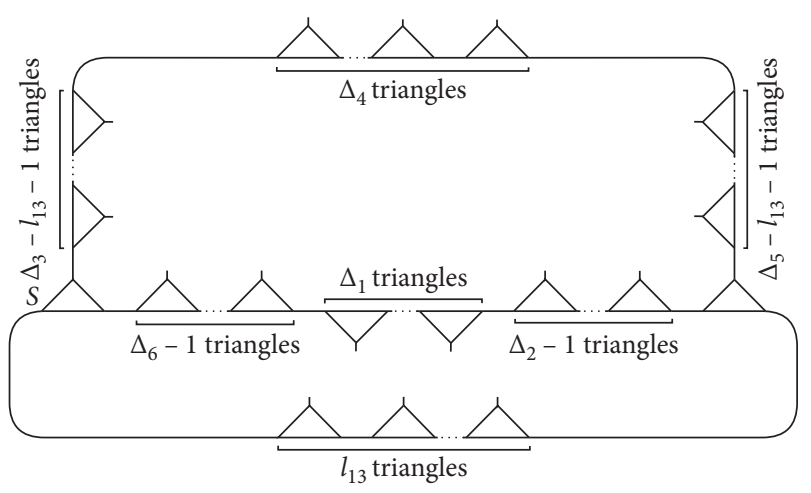

FIGURE 25: Homomorphic images $\Omega_{l_{13}^{17}}^{17}$.

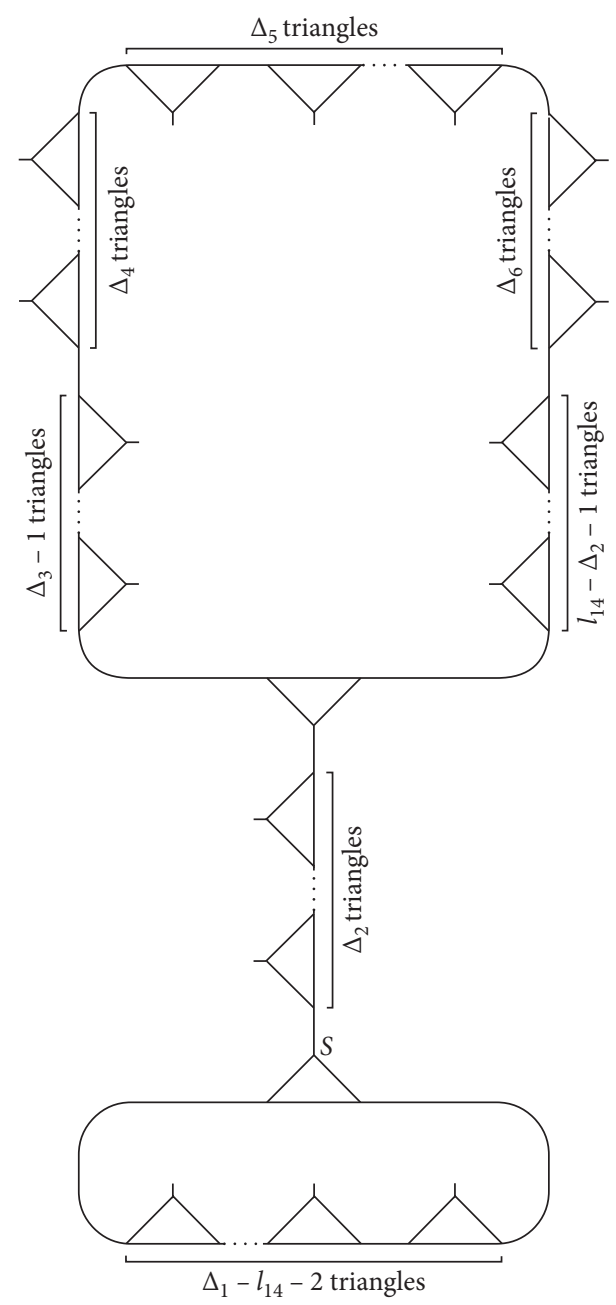

FIgURE 26: Homomorphic images $\Omega_{l_{14}}^{18}$.

one is the mirror image of itself. This lemma can be proved by using the same procedure as that for Lemma 18.

Let $k_{2} \in\{2,3\}$.

Lemma 23. If we contract the vertices $e_{3 l_{4}+1}^{1}: l_{4}=1,2, \ldots$, $\Delta_{1}-1$, with the vertices $e_{3 l_{\left(5, k_{2}\right)}}^{k_{2}}: l_{\left(5, k_{2}\right)}=1,2, \ldots, \Delta_{k_{2}}-1$, in the circuit $\Delta$, then for each $k_{2}$, there arise $\left(\Delta_{1}-1\right)\left(\Delta_{k_{2}}-1\right)$ distinct homomorphic images of $\Delta$ and 6 pairs of contracted vertices create each homomorphic image. Moreover, the number of total pairs of contracted vertices to generate all $\left(\Delta_{1}-1\right)\left(\Delta_{k}-1\right)$ and their mirror homomorphic images of $\Delta$ is $12\left(\Delta_{1}-1\right)\left(\Delta_{k_{2}}-1\right)$.

Proof. For $k_{2}=2$, let $H_{23}^{2}=\left\{\Omega_{\left(l_{4}, l_{(5,2)}\right.}^{23} ; l_{4}=1,2, \ldots\right.$, $\left.\Delta_{1}-1 ; l_{(5,2)}=1,2, \ldots, \Delta_{2}-1\right\}$ (Figure 33 ) be the collection of homomorphic images of $\Delta$ acquired by the contraction of the vertices $e_{3 l_{4}+1}^{1}$ with the vertex $e_{3 l_{(5,2)}}^{2}$ in $\Delta$, where $e_{3 l_{4}+1}^{1}$ and $e_{3 l_{(5,2)}}^{2}$ are fixed by the words $(p q)^{3 l_{4}}\left(p q^{-1}\right)^{\Delta_{6}}(p q)^{\Delta_{5}}\left(p q^{-1}\right)^{\Delta_{4}}(p q)^{\Delta_{3}}\left(p q^{-1}\right)^{\Delta_{2}}(p q)^{\Delta_{1}-l_{4}}$ and $\left(p q^{-1}\right) \quad \Delta_{2}-l_{(5,2)}(p q)^{\Delta_{1}}\left(p q^{-1}\right)^{\Delta_{6}}(p q)^{\Delta_{5}}$ $\left(p q^{-1}\right)^{\Delta_{4}}(p q)^{\Delta_{3}}\left(p q^{-1}\right)^{l_{(5,2)}}(p q)^{\Delta_{5}}\left(p q^{-1}\right)^{\Delta_{4}}(p q)^{\Delta_{3}}\left(p q^{-1}\right)^{l_{(5,2)}}$. It is easy to verify that $P_{1}=\left(p q^{-1}\right)^{\Delta_{2}-l_{(5,2)}}(p q)^{\Delta_{1}-l_{4}}$ and $P_{2}=$ $(p q)^{l_{4}}\left(p q^{-1}\right)^{\Delta_{6}}(p q)^{\Delta_{5}}\left(p q^{-1}\right)^{\Delta_{4}}(p q)^{\Delta_{3}}\left(p q^{-1}\right)^{l_{(5,2)}}$ are the possible paths between $e_{3 l_{4}+1}^{1}$ and $e_{3 l^{2}}^{2}$ (Figure 4 ). This implies that the homomorphic image $\Omega_{\left(l_{4}, l_{(5,2)}\right)}^{2,3)}$ has a vertex $s$ fixed by $P_{1}$ and $P_{2}$. Thus,

$$
E_{23}^{2}=\left\{e, q, q^{-1}, p, p q, p q^{-1}\right\}
$$

is the family of elements in $\operatorname{PSL}(2, \mathbb{Z})$ such that $\forall x \in E_{23}^{2}, \Delta$ contains the vertices $\left(e_{3 l_{4}+1}^{1}\right) x$ and $\left(e_{3 l_{(5,2)}}^{2}\right) x$. This gives that the cardinality of $E_{23}^{2}$, that is, 6 is the number of contracted pairs of vertices to produce the homomorphic image $\Omega_{\left(l_{4}, l_{(5,2)}\right)}^{23}$ (Theorem 2).

Now, we will prove that all the homomorphic images in $H_{23}^{2}$ are distinct and no one is the mirror image of itself.

Let $\Omega_{\left(m_{1}, m_{2}\right)}^{23}$ and $\Omega_{\left(n_{1}, n_{2}\right)}^{23}$ be any two elements of $H_{23}^{2}$, then $\Omega_{\left(m_{1}, m_{2}\right)}^{23}$ is produced by contraction of $e_{3 m_{1}+1}^{1}$ and $e_{3 m_{2}}^{2}$ and $\Omega_{\left(n_{1}, n_{2}\right)}^{23^{3}}$ is produced by contraction of $e_{3 n_{1}+1}^{1}$ and $e_{3 n_{2}}^{2}$.

Suppose $\Omega_{\left(m_{1}, m_{2}\right)}^{23}$ and $\Omega_{\left(n_{1}, n_{2}\right)}^{23}$ are the same. This concludes that $\Omega_{\left(m_{1}, m_{2}\right)}^{23}$ is procurable also by contracting $e_{3 n_{1}+1}^{1}$ and $e_{3 n_{2}}^{2}$, implying that $e_{3 n_{1}+1}^{1} \leftrightarrow e_{3 n_{2}}^{2}$ is one of the pairs of contracted vertices for $\Omega_{\left(m_{1}, m_{2}\right)}^{23}$. Then there must exist an element $w \in E_{23}^{2} \quad$ such that $\left(e_{3 m_{1}+1}^{1}\right) w=e_{3 n_{1}+1}^{1}$ and $\left(e_{3 m_{2}}^{2}\right) w=e_{3 n_{2}}^{2} \cdot p q^{-1} \in E_{5}^{2}$ is the only element such that $\left(e_{3 m_{2}}^{2}\right) p q^{-1}=e_{3\left(m_{2}+1\right)}^{2}$ but $\left(e_{3 m_{1}+1}^{1}\right) p q^{-1} \neq e_{3 n_{1}+1}^{1}$. Thus, $\Omega_{\left(m_{1}, m_{2}\right)}^{23}$ and $\Omega_{\left(n_{1}, n_{2}\right)}^{23}$ are not the same, that is, by contracting $e_{3 m_{1}+1}^{1}$ and $e_{3 m_{2}}^{2}$ to produce $\Omega_{\left(m_{1}, m_{2}\right)}^{23}, e_{3 n_{1}+1}^{1}$ and $e_{3 n_{2}}^{2}$ are not contracted. Now, if $\Omega_{\left(m_{1}, m_{2}\right)}^{23}$ and $\Omega_{\left(n_{1}, n_{2}\right)}^{23 *}$ are the same, then there must exist an element $w \in E_{23}^{2}$ such that $\left(e_{3 m_{1}+1}^{1}\right) w=$ $e_{3 n_{1}+1}^{1 *}$ and $\left(e_{3 m_{2}}^{2}\right) w=e_{3 n_{2}}^{2 *} \cdot p \in E_{23}^{2}$ is the only element such that $\left(e_{3 m_{1}+1}^{1}\right) p=e_{3\left(\Delta_{1}-m_{1}\right)+1}^{1 *}$ but $\left(e_{3 m_{2}}^{2}\right) p \neq e_{3 m_{2}}^{2 *}$. This implies that $\Omega_{\left(m_{1}, m_{2}\right)}^{23}$ and $\Omega_{\left(n_{1}, n_{2}\right)}^{23}$ are not the mirror images of each other, that is, by contracting $e_{3 m_{1}+1}^{1}$ and $e_{3 m_{2}}^{2}$ to produce $\Omega_{\left(m_{1}, m_{2}\right)}^{23}, e_{3 n_{1}+1}^{1 *}$ and $e_{3 n_{2}}^{2 *}$ are not contracted. Hence, all the homomorphic images in $H_{23}^{2}$ are distinct. Thus, $H_{23}^{2}=\left\{\Omega_{\left(l_{4}, l_{(5,2)}\right)}^{23} ; l_{4}=1,2, \ldots, \Delta_{1}-1 ; l_{(5,2)}=1,2, \ldots, \Delta_{2}-\right.$ $1\}$ gives $\left|H_{23}^{2}\right|=\left(\Delta_{1}-1\right)\left(\Delta_{2}-1\right)$.

Suppose $\Omega_{\left(m_{1}, m_{2}\right)}^{23}$ is the mirror image of itself, then there must exist an element $w \in E_{23}^{2}$ such that $\left(e_{3 m_{1}+1}^{1}\right) w=e_{3 m_{1}+1}^{1 *}$ and $\left(e_{3 m_{2}}^{2}\right) w=e_{3 m_{2}}^{2 *} \cdot p \in E_{23}^{2}$ is the only element such that 


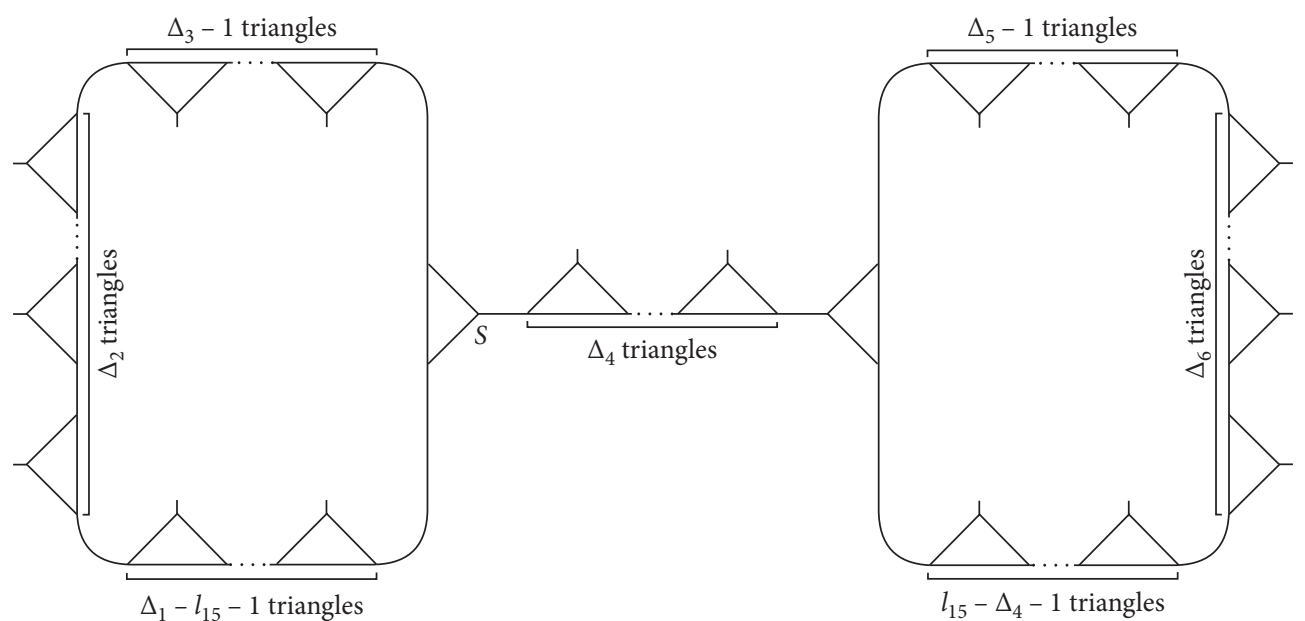

FIgURE 27: Homomorphic images $\Omega_{l_{15}}^{19}$.

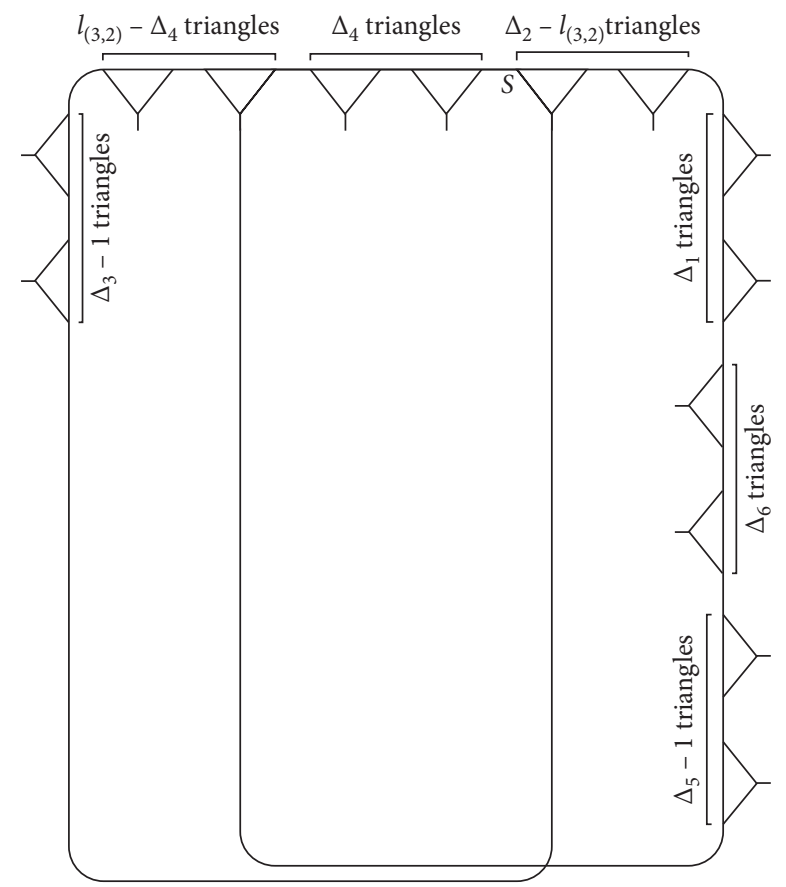

FIGURE 28: Homomorphic images $\Omega_{l_{(3,2)}^{20}}^{2}$.

$\left(e_{3 m_{1}+1}^{1}\right) p=e_{3\left(\Delta_{1}-m_{1}\right)+1}^{1 *}$ but $\left(e_{3 m_{2}}^{2}\right) p \neq e_{3 m_{2}}^{2 *}$. This implies that $\Omega_{\left(m_{1}, m_{2}\right)}^{23}$ is not the same as $\Omega_{\left(m_{1}, m_{2}\right)}^{23}$, that is, by contracting $e_{3 m_{1}+1}^{1}$ and $e_{3 m_{2}}^{2}$ to produce $\Omega_{\left(m_{1}, m_{2}\right)}^{23}, e_{3 m_{1}+1}^{1 *}$ and $e_{3 m_{2}}^{2 *}$ are not contracted.

Hence, there are

$$
2 \times\left|E_{23}^{2}\right| \times\left|H_{23}^{2}\right|=12\left(\Delta_{1}-1\right)\left(\Delta_{2}-1\right)
$$

pairs of contracting vertices to produce all the homomorphic images in $H_{23}^{2}$.

We can prove this lemma for $k_{2}=3$ (Figure 34) in similar way as that for $k_{2}=2$.

$$
\begin{aligned}
& \text { Let } \quad \eta_{2}=\left\{\begin{array}{ll}
0 & \text { if } \Delta_{4}=0(\bmod 2) \\
1 & \text { otherwise }
\end{array} \eta_{3}=\left\{\begin{array}{ll}
0 & \text { if } \Delta_{1}=0(\bmod 2) \\
1 & \text { otherwise }
\end{array} .\right.\right.
\end{aligned}
$$$$
\text { and }
$$

Lemma 24. If we contract the vertices $e_{3 l_{17}+1}^{1}: l_{17}= \begin{cases}1,2, \ldots,\left(\left(\Delta_{1}-\eta_{3}\right) / 2\right), & \text { if } l_{18}=\left(\Delta_{4} / 2\right), \text { with } \\ 1,2, \ldots, \Delta_{1}-1 & \text { otherwise }\end{cases}$ the vertices $e_{3 l_{18}}^{4}: l_{18}=1,2, \ldots,\left(\left(\Delta_{4}-\eta_{2}\right) / 2\right)$, in the circuit $\Delta$, then there arise $\left\{\begin{array}{ll}(1 / 2)\left(\Delta_{1}-1\right)\left(\Delta_{4}-1\right) & \text { if } \Delta_{4}=1(\bmod 2) \\ (1 / 2)\left[\left(\Delta_{1}-1\right)\left(\Delta_{4}-1\right)+1-\eta_{3}\right] & \text { if } \Delta_{4}=0(\bmod 2)\end{array}\right.$ distinct homomorphic images of $\Delta$ and 6 pairs of contracted vertices create each homomorphic image. Moreover, the number of total pairs of contracted vertices to generate all these homomorphic images and their mirror homomorphic images of $\Delta$ is $6\left(\Delta_{1}-1\right)\left(\Delta_{4}-1\right)$.

Proof. Let $H_{24}=\left\{\Omega_{(l} \quad{ }_{17}, l_{18}\right)^{24} ; l_{17}=\{1,2, \ldots$, $\left(\left(\Delta_{1}-\eta_{3}\right) / 2\right)$, if $l_{18}=\left(\Delta_{4} / 2\right), l_{18}=1,2, \ldots,\left(\left(\Delta_{4}-\right.\right.$

$\left.\left.\eta_{2}\right) / 2\right) 1,2, \ldots, \Delta_{1}-1$ otherwise (Figure 35) be the collection of homomorphic images of $\Delta$ acquired by the contraction of the vertices $e_{3 l_{17}+1}^{1}$ with the vertices $e_{3 l_{18}}^{4}$ in $\Delta$, where $e_{3 l_{17}+1}^{1}$ and $e_{3 l^{4}}^{4}$ are fixed by the words $(p q)^{l_{17}}\left(p q^{-1}\right)^{\Delta_{6}}(p q)^{\Delta_{5}}\left(p q^{-1}\right)^{\Delta_{4}}(p q)^{\Delta_{3}}\left(p q^{-1}\right)^{\Delta_{2}}(p q)^{\Delta_{1}-l_{18}}$ and $\left(p q^{-1}\right)^{\Delta_{4}-l_{18}}(p q)^{\Delta_{3}}\left(p q^{-1}\right)$ $\Delta_{2}(p q)^{\Delta_{1}}\left(p q^{-1}\right)^{\Delta_{6}}(p q)^{\Delta_{5}}\left(p q^{-1}\right)^{l_{18}}$. It is easy to verify that $P_{1}=(p q)^{l_{17}}\left(p q^{-1}\right)^{\Delta_{6}}(p q)^{\Delta_{5}}\left(p q^{-1}\right)^{l_{18}} \quad$ and $P_{2}=\left(p q^{-1}\right)^{\Delta_{4}-l_{18}}(p q)^{\Delta_{3}}\left(p q^{-1}\right)^{\Delta_{2}}(p q)^{\Delta_{1}-l_{17}}$ are the possible paths between $e_{3 l_{17}+1}^{1}$ and $e_{3 l_{18}^{4}}^{4}$ (Figure 4). This implies that the homomorphic image $\Omega_{\left(l_{17}, l_{18}\right)}^{24}$ has a vertex $s$ fixed by $P_{1}$ and $P_{2}$. Thus,

$$
E_{24}=\left\{e, q, q^{-1}, p, p q, p q^{-1}\right\}
$$

is the family of elements in $\operatorname{PSL}(2, \mathbb{Z})$ such that $\forall x \in E_{24}, \Delta$ contains the vertices $\left(e_{3 l_{17}+1}^{1}\right) x$ and $\left(e_{3 l_{18}}^{4}\right) x$. This gives that the cardinality of $E_{24}$, that is, 6 is the number of contracted pairs of vertices to produce the homomorphic image $\Omega_{\left(l_{17}, l_{18}\right)}^{24}$ (Theorem 2).

Now, we prove that $(i)$ for $\Delta_{4}=0(\bmod 2)$, all the elements in $\mathrm{H}_{24}$ are distinct and no one is the mirror image of itself, and (ii) for $\Delta_{4}=1(\bmod 2)$, all the elements in $H_{24}$ are distinct and the homomorphic image $\Omega_{\left(\left(\Delta_{1} / 2\right),\left(\Delta_{4} / 2\right)\right)}^{24}$ is the mirror image of itself. 


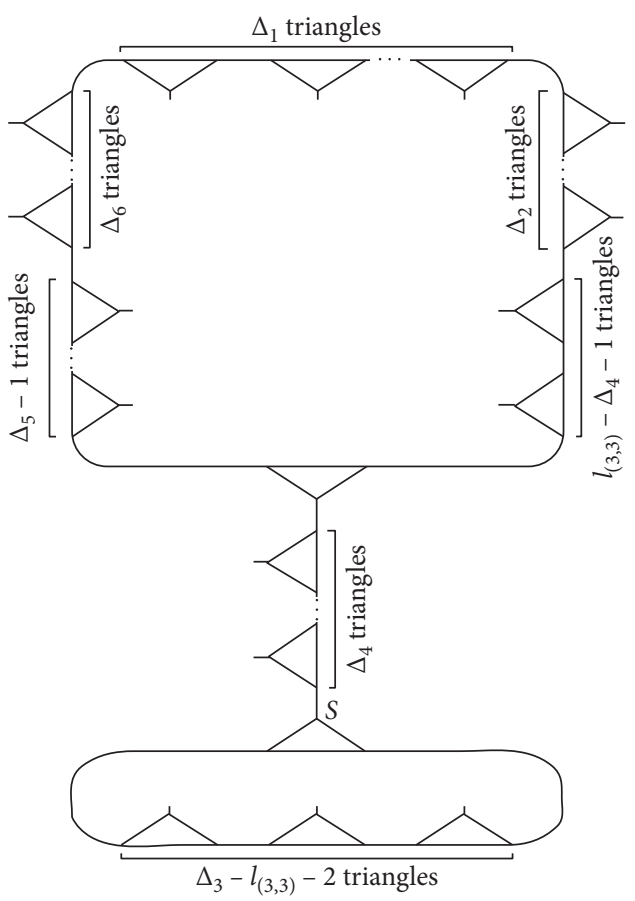

FIGURE 29: Homomorphic images $\Omega_{l_{(3,3)}^{20}}^{2}$

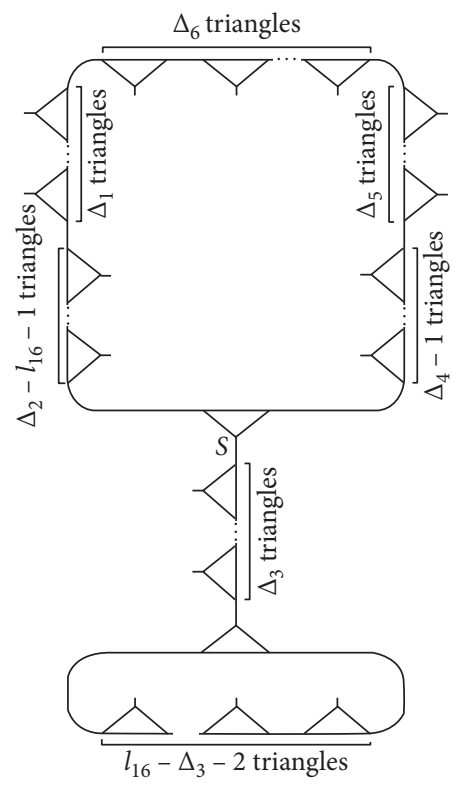

FIgURE 30: Homomorphic images $\Omega_{l_{16}}^{21}$.

Suppose $\Omega_{\left(m_{1}, m_{2}\right)}^{24}$ and $\Omega_{\left(n_{1}, n_{2}\right)}^{24}$ are the same. This concludes that $\Omega_{\left(m_{1}, m_{2}\right)}^{24}$ is procurable also by contracting $e_{3 n_{1}+1}^{1}$ and $e_{3 n_{2}}^{4}$, implying that $e_{3 n_{1}+1}^{1} \leftrightarrow e_{3 n_{2}}^{4}$ is one of the pairs of contracted vertices for $\Omega_{\left(m_{1}, m_{2}\right)}^{24}$. Then there must exist an element $w \in E_{24}$ such that $\left(e_{3 m_{1}+1}^{1}\right) w=e_{3 n_{1}+1}^{1}$ and $\left(e_{3 m_{2}}^{4}\right) w=e_{3 n_{2}}^{4} \cdot p q^{-1} \in E_{24}$ is the only element such that $\left(e_{3 m_{2}}^{4}\right) p q^{-1}=e_{3\left(m_{2}+1\right)}^{4} \quad$ but $\left(e_{3 m_{1}+1}^{1}\right) p q^{-1} \neq e_{3 n_{1}+1}^{1}$. Thus, $\Omega_{\left(m_{1}, m_{2}\right)}^{24}$ and $\Omega_{\left(n_{1}, n_{2}\right)}^{24}$ are not the same, that is, by contracting $e_{3 m_{1}+1}^{1}$ and $e_{3 m_{2}}^{4}$ to produce $\Omega_{\left(m_{1}, m_{2}\right)}^{24}, e_{3 n_{1}+1}^{1}$ and $e_{3 n_{2}}^{4}$ are not contracted. Now, if $\Omega_{\left(m_{1}, m_{2}\right)}^{24}$ and $\Omega_{\left(n_{1}, n_{2}\right)}^{24}$ are the same, then there must exist an element $w \in E_{24}$ such that $\left(e_{3 m_{1}+1}^{1}\right) w=$ $e_{3 n_{1}+1}^{1 *}$ and $\left(e_{3 m_{2}}^{4}\right) w=e_{3 n_{2}}^{4 *} \cdot p \in E_{24}$ is the only element such that $\left(e_{3 m_{1}+1}^{1}\right) p=e_{3\left(\Delta_{1}-m_{1}\right)+1}^{1 *}$ and $\left(e_{3 m_{2}}^{4}\right) p=e_{3\left(\Delta_{4}-m_{2}\right)}^{4 *}$ This implies that $\Omega_{\left(m_{1}, m_{2}\right)}^{24}$ and $\Omega_{\left(n_{1}, n_{2}\right)}^{24}$ are the mirror images of each other if and only if $n_{1}=\Delta_{1}-m_{1}$ and $n_{2}=\Delta_{4}-m_{2}$, that is, by contracting $e_{3 m_{1}+1}^{1}$ and $e_{3 m_{2}}^{4}$ to produce $\Omega_{\left(m_{1}, m_{2}\right)}^{24}, e_{3 n_{1}+1}^{1 *}$ and $e_{3 n_{2}}^{4 *}$ are also contracted and the homomorphic image $\Omega_{\left(m_{1}, m_{2}\right)}^{24}$ is the mirror image of itself if and only if $m_{1}=$ $\Delta_{1}-m_{1}$ and $m_{2}=\Delta_{4}-m_{2}$ that is $m_{1}=\left(\Delta_{1} / 2\right)$ and $m_{2}=\left(\Delta_{4} / 2\right)$. Now,

(1)If $\Delta_{4}=1(\bmod 2)$ then $\forall m_{2} \in l_{18}$, we get $\Delta_{4}-m_{2}>\left(\left(\Delta_{4}-1\right) / 2\right)$ implies $\Omega_{\left(\Delta_{1}-m_{1}, \Delta_{4}-m_{2}\right)}^{24} H_{24}$. This indicates that no homomorphic image in $H_{24}$ is the mirror image of other. Hence, all the elements in $H_{24}$ are distinct. Thus, $H_{24}=\left\{\Omega_{\left(l_{17}, l_{18}\right)}{ }^{24} ; l_{17}=\{1,2\right.$, $\ldots,\left(\left(\Delta_{1}-\eta_{3}\right) / 2\right) \quad$ if $l_{18}=\left(\Delta_{4} / 2\right), \quad l_{18}=1,2, \ldots$, $\left(\left(\Delta_{4}-\eta_{2}\right) / 2\right) 1,2, \ldots, \Delta_{1}-1$ otherwise $\}, \quad$ implying that

$$
\left|H_{24}\right|=\frac{1}{2}\left(\Delta_{1}-1\right)\left(\Delta_{4}-1\right) .
$$

Also, $\left(\Delta_{4} / 2\right)>\left(\left(\Delta_{4}-1\right) / 2\right)$ implies no homomorphic image in $\mathrm{H}_{24}$ is the mirror image of itself. Hence, there are

$$
2 \times\left|E_{24}\right| \times\left|H_{24}\right|=6\left(\Delta_{1}-1\right)\left(\Delta_{4}-1\right)
$$

pairs of contracting vertices to produce all the homomorphic images in $\mathrm{H}_{24}$.

(2) If $\Delta_{4}=0(\bmod 2)$, then only for $m_{2}=\left(\Delta_{4} / 2\right), \Delta_{4}-$ $m_{2}=\left(\Delta_{4} / 2\right) \in l_{18} \quad$ and $\quad \forall m_{1} \in l_{17}\left\{\Delta_{1} / 2\right\}$, $\Delta_{1}-m_{1} \notin l_{17}$, implying that $\Omega_{\left(\Delta_{1}-m_{1},\left(\Delta_{4} / 2\right)\right)}^{24} \notin H_{24}$. Hence, all the elements in $H_{24}$ are distinct. Thus, $H_{24}=\left\{\Omega_{\left(l_{17},\right.} \quad l_{18}\right)^{24} ; l_{17}=\left\{1,2, \quad \ldots,\left(\left(\Delta_{1}-\right.\right.\right.$ $\left.\left.\eta_{3}\right) / 2\right)$ if $l_{18}=\left(\Delta_{4} / 2\right) ; \quad l_{18}=1,2, \ldots,\left(\left(\Delta_{4}-\eta\right.\right.$ $\left.\left.{ }_{2}\right) / 2\right) 1,2, \ldots, \Delta_{1}-1$ otherwise $\}$, implying that

$$
\begin{aligned}
\left|H_{24}\right| & =\frac{1}{2}\left(\Delta_{1}-1\right)\left(\Delta_{4}-2\right)+\frac{1}{2}\left(\Delta_{1}-\eta_{3}\right) \\
& =\frac{1}{2}\left[\left(\Delta_{1}-1\right)\left(\Delta_{4}-1\right)+1-\eta_{3}\right] .
\end{aligned}
$$

Now,

(a). If $\Delta_{1}$ is odd, then $\left(\Delta_{1} / 2\right) \notin l_{17}$, implying that $\Omega_{\left(\left(\Delta_{1} / 2\right),(4 / 2)\right)}^{24} \notin H_{24}$. So, no homomorphic image in $\mathrm{H}_{24}$ is the mirror image of itself. Hence, there are

$$
2 \times\left|E_{24}\right| \times\left|H_{24}\right|=6\left(\Delta_{1}-1\right)\left(\Delta_{4}-1\right)
$$

pairs of contracting vertices to produce all the homomorphic images in $\mathrm{H}_{24}$.

(b) If $\Delta_{1}$ is even, then $\left(\Delta_{1} / 2\right) \in l_{17}$, implying that $\Omega_{\left(\left(\Delta_{1} / 2\right),\left(\Delta_{4} / 2\right)\right)}^{24} \in H_{24}$ is the mirror image of itself. Hence, there are

$2 \times\left|E_{24}\right| \times\left(\left|H_{24}\right|-1\right)+\left|E_{24}\right|=6\left(\Delta_{1}-1\right)\left(\Delta_{4}-1\right)$

pairs of contracting vertices to produce all the homomorphic images in $\mathrm{H}_{24}$. 


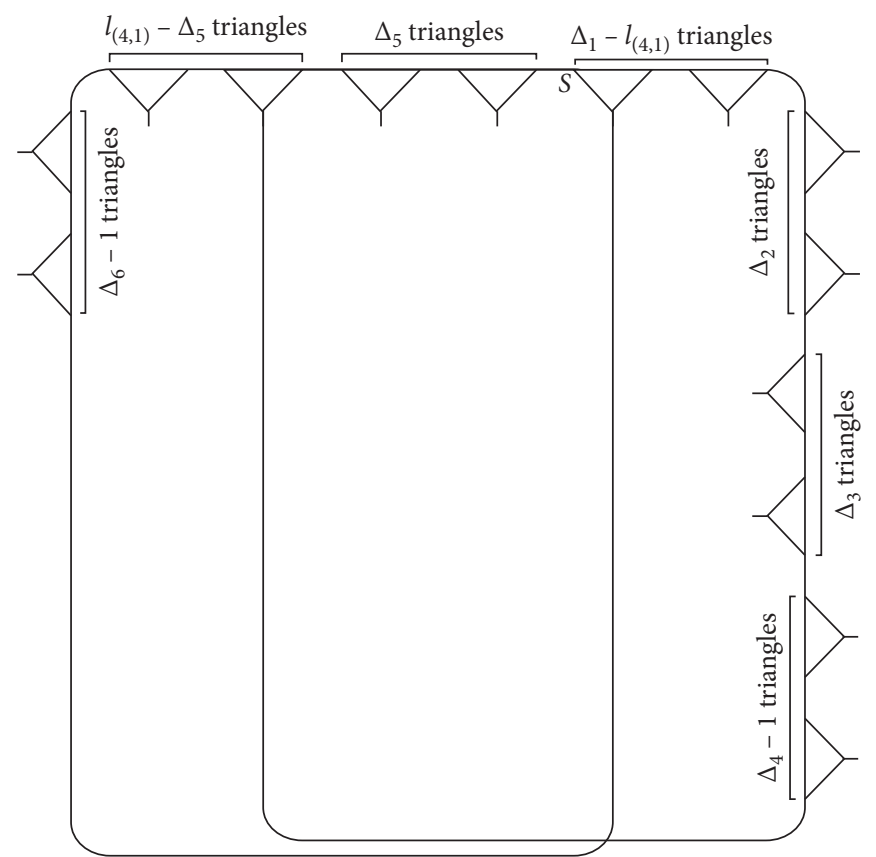

FIGURE 31: Homomorphic images $\Omega_{l_{(4,1)}^{22}}^{2}$.

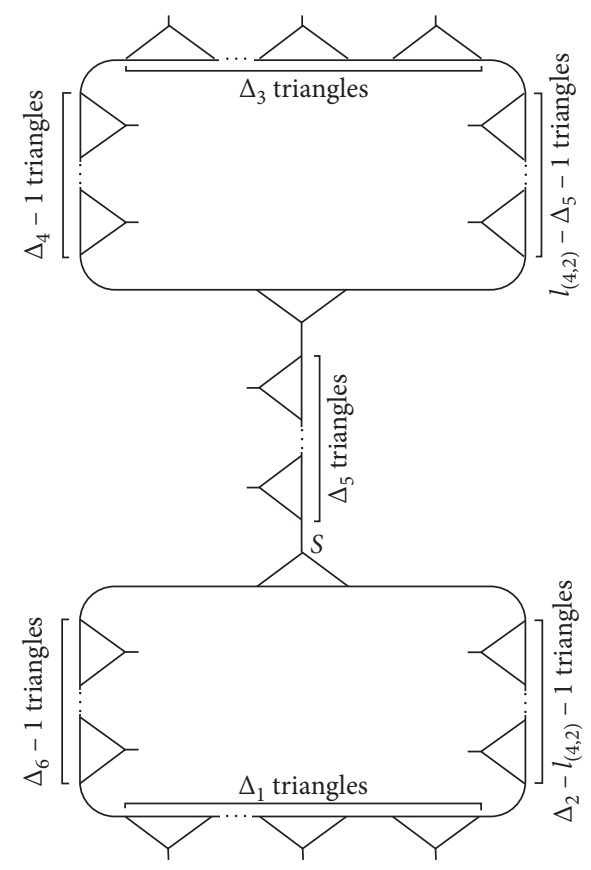

FIGURE 32: Homomorphic images $\Omega_{l_{(4,2)}^{22}}$.

Let $k_{1} \in\{3,4,5\}$.

Lemma 25. If we contract the vertices $e_{3 l_{5}+1}^{2}: l_{5}=1,2, \ldots$, $\Delta_{2}-1$, with the vertices $e_{3 l_{\left(6, k_{1}\right)}}^{k_{1}} l_{\left(6, k_{1}\right)}=1,2, \ldots, \Delta_{k_{1}}-1$, in the circuit $\Delta$, then for each $k_{5}$, there arise $\left(\Delta_{2}-1\right)\left(\Delta_{k_{1}}-1\right)$ distinct homomorphic images of $\Delta$ and 6 pairs of contracted vertices create each homomorphic image. Moreover, the number of total pairs of contracted vertices to generate all $\left(\Delta_{2}-1\right)\left(\Delta_{k_{1}}-1\right)$ and their mirror homomorphic images of $\Delta$ is $12\left(\Delta_{2}-1\right)\left(\Delta_{k_{1}}-1\right)$.

For a fix value of $k_{1}$, let $H_{25}^{k_{1}}=\left\{\Omega_{\left(l_{5}, l_{\left(6, k_{1}\right)}\right)}^{25} ; l_{5}=\right.$ $\left.1,2, \ldots, \Delta_{2}-1 ; l_{\left(6, k_{1}\right)}=1,2, \ldots, \Delta_{k_{1}}-1\right\}$ be the collection of homomorphic images of $\Delta$ acquired by the contraction of the vertices $e_{3 l_{5}+1}^{2}$ with the vertices $e_{3 l_{\left(6, k_{1}\right)}}^{k_{1}}$ in $\Delta$. Figures 36,37 , and 


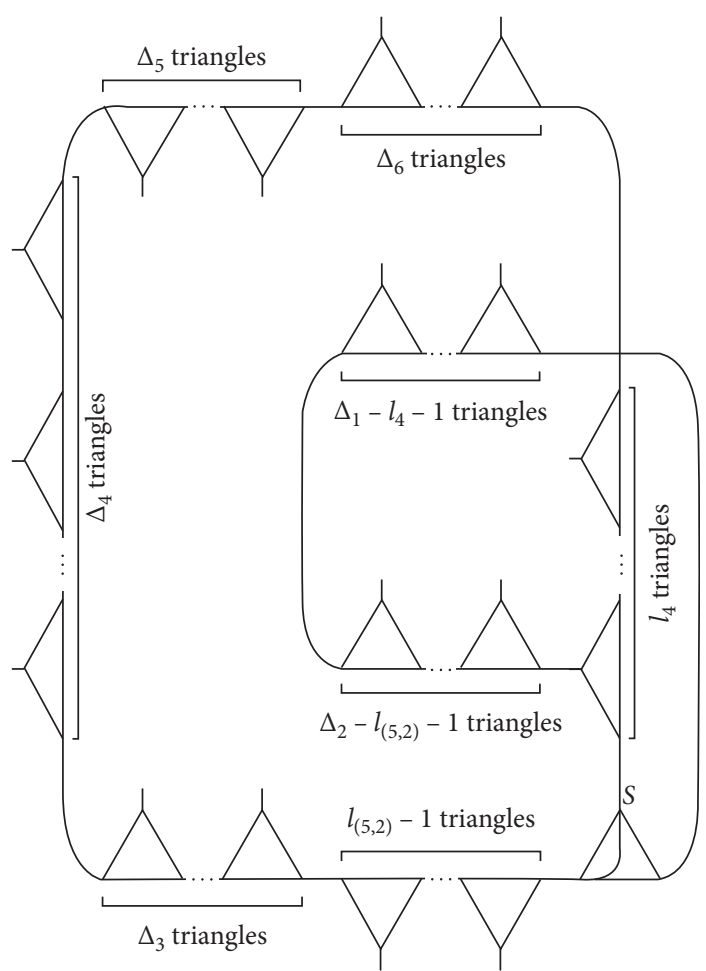

FIgURE 33: Homomorphic images $\Omega_{\left(l_{4}, l_{(5,2)}\right)}^{23}$.

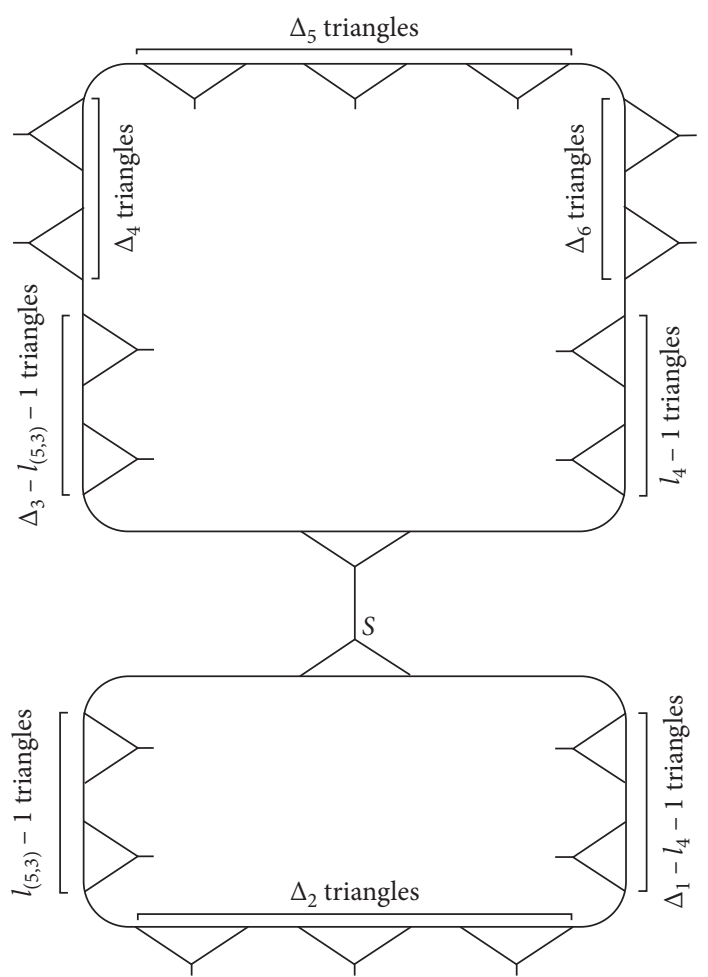

FIgURE 34: Homomorphic images $\Omega_{\left(l_{4}, l_{(5,3)}\right.}^{23}$. 


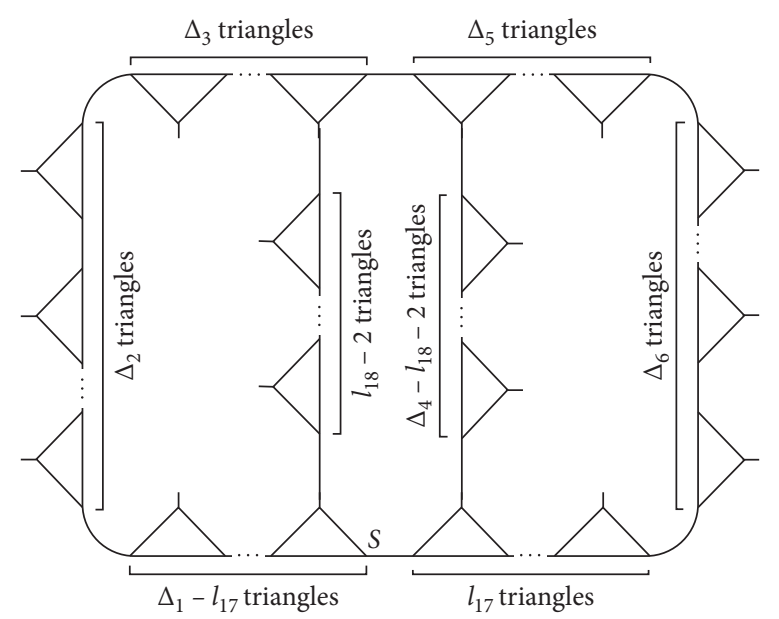

FIgURE 35: Homomorphic images $\Omega_{\left(l_{17}, l_{18}\right)}^{24}$.

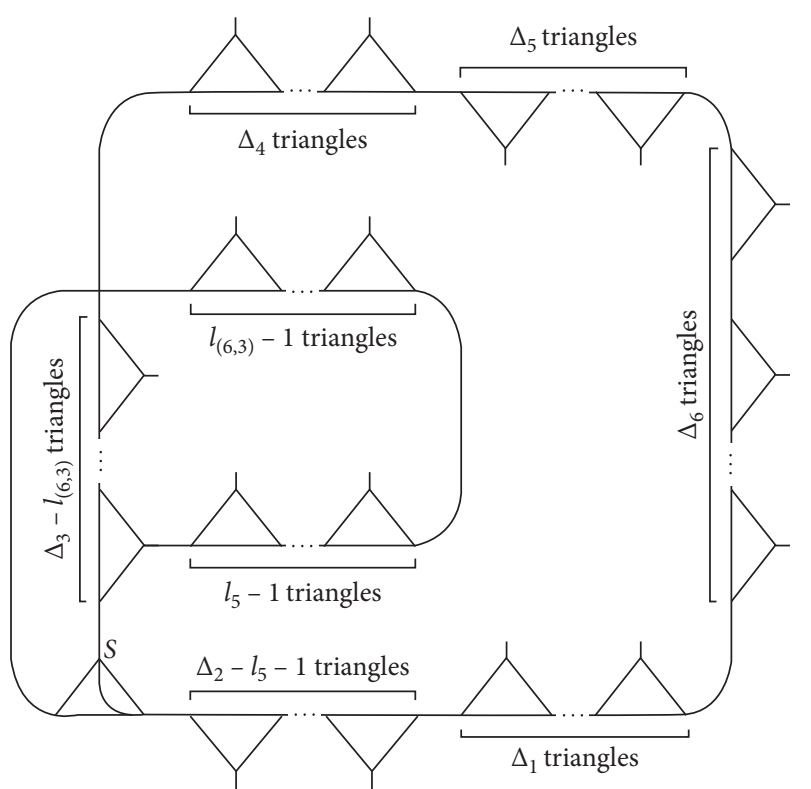

FIGURE 36: Homomorphic images $\Omega_{\left(l_{5}, l_{(6,3)}\right)}^{25}$.

38 present $H_{25}^{k_{1}}$ graphically. From all the homomorphic images presented in these figures, it is not intricated to check that no one is the mirror image of itself.

This lemma can be proved by using the same procedure as that for Lemma 23.

Lemma 26. If we contract the vertices $e_{3 l_{13}+1}^{3}: l_{13}=1,2, \ldots$, $\Delta_{3}-1$, with the vertices $e_{3 l_{7}}^{4}: l_{7}=1,2, \ldots, \Delta_{4}-1$, in the circuit $\Delta$, then there arise $\left(\Delta_{3}-1\right)\left(\Delta_{4}-1\right)$ distinct homomorphic images of $\Delta$ and 6 pairs of contracted vertices create each homomorphic image. Moreover, the number of total pairs of contracted vertices to generate all $\left(\Delta_{3}-1\right)\left(\Delta_{4}-1\right)$ and their mirror homomorphic images of $\Delta$ is $12\left(\Delta_{3}-1\right)\left(\Delta_{4}-1\right)$.

Let $H_{26}=\left\{\Omega_{\left(l_{13}, l_{7}\right)}^{26} ; l_{13}=1,2, \ldots, \Delta_{3}-1 ; l_{7}=1,2, \ldots\right.$, $\left.\Delta_{4}-1\right\}$ be the collection of homomorphic images of $\Delta$ acquired by the contraction of the vertices $e_{3 l_{13}+1}^{3}$ with the vertices $e_{3 l_{7}}^{4}$ in $\Delta$. Figure 39 presents $H_{26}$ graphically. From all the homomorphic images presented in Figure 39, it is not intricated to check that no one is the mirror image of itself. This lemma can be proved by using the same procedure as that for Lemma 23.

$$
\text { Let } \overline{k_{2}}=8-k_{2} \text {. }
$$

Lemma 27. If we contract the vertices $e_{3 l_{\left(7, k_{2}\right)}+1}^{k_{k}}: l_{\left(7, k_{2}\right)}=$

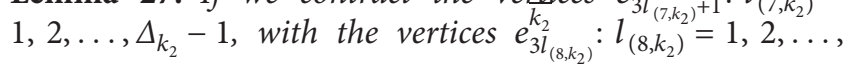
$\Delta_{k_{2}}-l_{\left(7, k_{2}\right)}$, in the circuit $\Delta$, then for each $k_{3}$, there arise $(1 / 2) \Delta_{k_{2}}\left(\Delta_{k_{2}}-1\right)$ distinct homomorphic images of $\Delta$ and 6 pairs of contracted vertices create each homomorphic image. Moreover, the number of total pairs of contracted vertices to generate all $(1 / 2) \Delta_{k_{2}}\left(\Delta_{k_{2}}-1\right)$ and their mirror homomorphic images of $\Delta$ is $6\left(\Delta_{k_{2}}-1\right)^{2}$.

Proof. For $k_{2}=2$, let $H_{27}^{2}=\left\{\Omega_{\left(l_{(7,2)}, l_{(8,2)}\right)}{ }^{27} ; l_{(7,2)}=1,2, \ldots\right.$, $\left.\Delta_{2}-1 ; l_{(8,2)}=1,2, \ldots, \Delta_{2}-l_{(7,2)}\right\}$ (Figure 40) be the collection of homomorphic images of $\Delta$ acquired by the contraction of the vertices $e_{3 l_{(7,2)}+1}^{2}$ with the vertex $e_{3 l_{(8,2)}}^{6}$ in $\Delta$, where $e_{3 l_{(7,2)}+1}^{2}$ and $e_{3 l_{(8,2)}}^{6}$ are fixed by the ${ }^{2(7,2)}$ words 


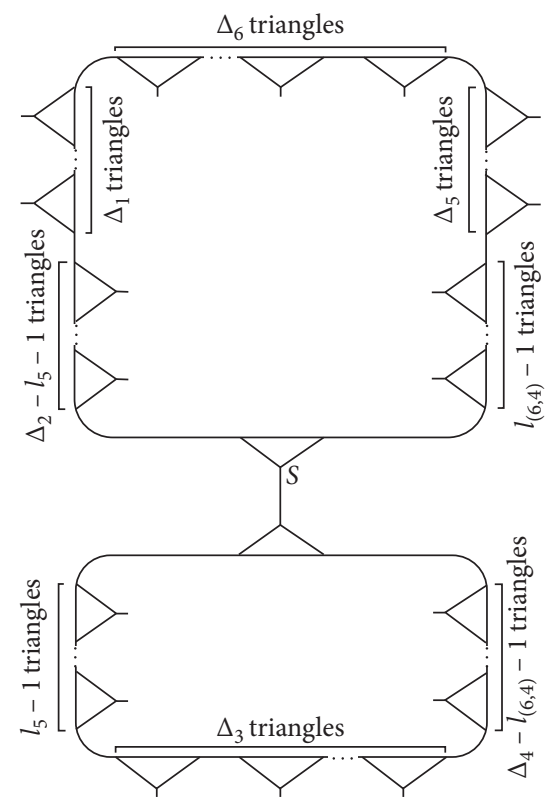

FIGURE 37: Homomorphic images $\Omega_{\left(l_{5}, l_{(6,4)}\right.}^{25}$.

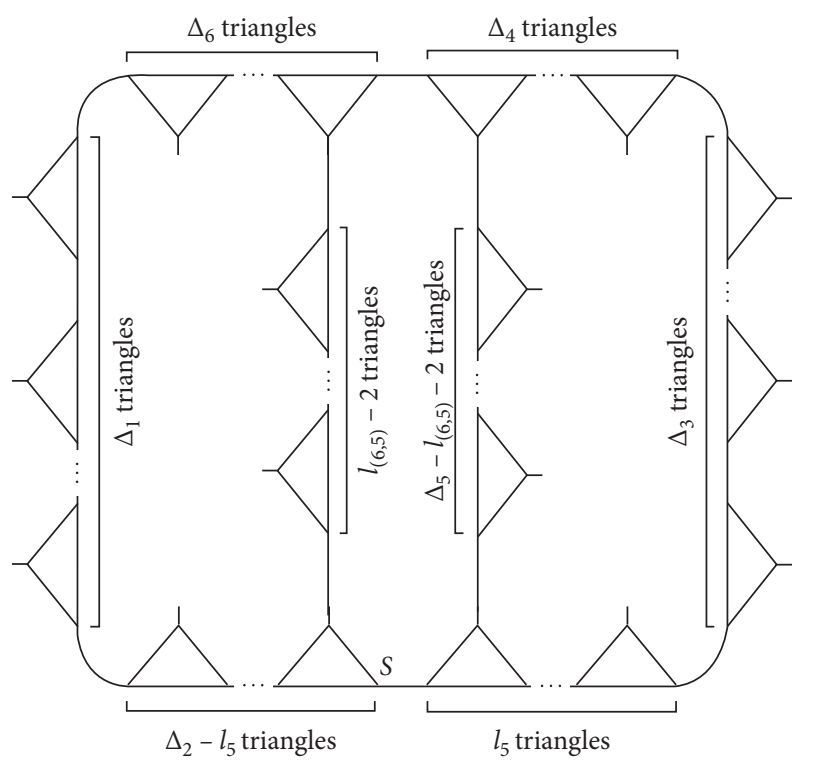

FIGURE 38: Homomorphic images $\Omega_{\left(l_{5}, l_{(6,5)}\right.}^{25}$.

$(p q)^{l_{(7,2)}}\left(p q^{-1}\right)^{\Delta_{3}}(p q)^{\Delta_{4}}\left(p q^{-1}\right)^{\Delta_{5}}(p q)^{\Delta_{6}}\left(p q^{-1}\right)^{\Delta_{1}}(p q)^{\Delta_{2}-l_{(7,2)}}$ and $\left(p q^{-1}\right)^{\Delta_{6}-l_{(8,2)}}(p q)^{\Delta_{5}}\left(p q^{-1}\right)^{\Delta_{4}}(p q)^{\Delta_{3}}\left(p q^{-1}\right)^{\Delta_{2}}(p q)^{\Delta_{1}}$ $\left(p q^{-1}\right)^{l_{(8,2)}}$. It is easy to verify that $P_{1}=(p q)^{l_{(7,2)}}$ $\left(p q^{-1}\right)^{\Delta_{3}}(p q)^{\Delta_{4}}\left(p q^{-1}\right)^{\Delta_{5}}(p q)^{\Delta_{6}-l_{(8,2)}} p$ and $P_{2}=q(p q)^{l_{(8,2)}-1}$ $\left(p q^{-1}\right)^{\Delta_{1}}(p q)^{\Delta_{2}-t_{(7,2)}}$ are the possible paths between $e_{3 l_{(7,2)}}^{2}$ and $e_{3 l}^{6} \quad$ (Figure 4 ). This implies that the homomorphic image $\Omega_{\left(l_{(7,2)}, l_{(8,2)}\right)}$ has a vertex $s$ fixed by $P_{1}$ and $P_{2}$. Thus,

$$
E_{27}^{2}=\left\{e, q, q^{-1}, p, p q, p q^{-1}\right\}
$$

is the family of elements in $\operatorname{PSL}(2, \mathbb{Z})$ such that $\forall w \in E_{27}^{2}, \Delta$ contains the vertices $\left(e_{3 l_{(72)}+1}^{2}\right) w$ and $\left(e_{3 l_{(8,2)}}^{6}\right) w$. This gives that the cardinality of $E_{27}^{2^{2}}$, that is, 6 is the number of contracted pairs of vertices to produce each homomorphic image $\Omega_{\left(l_{(7,2)}, l_{(8,2)}\right)}^{27}$ (Theorem 2).
Suppose $\Omega_{\left(m_{1}, m_{2}\right)}^{27}$ and $\Omega_{\left(n_{1}, n_{2}\right)}^{27}$ are the same. This concludes that $\Omega_{\left(m_{1}, m_{2}\right)}^{27}$ is procurable also by contracting $e_{3 n_{1}+1}^{2}$ and $e_{3 n_{2}}^{6}$, implying that $e_{3 n_{1}+1}^{2} \leftrightarrow e_{3 n_{2}}^{6}$ is one of the pairs of contracted vertices for $\Omega_{\left(m_{1}, m_{2}\right)}^{27^{1}}$. Then there must exist an element $w \in E_{27}^{2} \quad$ such that $\left(e_{3 m_{1}+1}^{2}\right) w=e_{3 n_{1}+1}^{2}$ and $\left(e_{3 m_{2}}^{6}\right) w=e_{3 n_{2}}^{6} \cdot p q^{-1} \in E_{27}^{2}$ is the only element such that $\left(e_{3 m_{2}}^{6}\right) p q^{-1}=e_{3\left(m_{2}+1\right)}^{6}$ but $\left(e_{3 m_{1}+1}^{2}\right) p q^{-1} \neq e_{3 n_{1}+1}^{2}$. Thus, $\Omega_{\left(m_{1}, m_{2}\right)}^{27}$ and $\Omega_{\left(n_{1}, n_{2}\right)}^{27}$ are not the same, that is, by contracting $e_{3 m_{1}+1}^{2}$ and $e_{3 m_{2}}^{6}$ to produce $\Omega_{\left(m_{1}, m_{2}\right.}^{27}, e_{3 n_{1}+1}^{2}$ and $e_{3 n_{2}}^{6}$ are not contracted. Now, if $\Omega_{\left(m_{1}, m_{2}\right)}^{27}$ and $\Omega_{\left(n_{1}, n_{2}\right)}^{27}$ are the same, then there must exist an element $w \in E_{27}^{2}$ such that $\left(e_{3 m_{1}+1}^{2}\right) w=$ $e_{3 n_{1}+1}^{2 *}$ and $\left(e_{3 m_{2}}^{6}\right) w=e_{3 n_{2}}^{6 *}$ or $\left(e_{3 m_{1}+1}^{2}\right) w=e_{3 n_{1}+1}^{6 *}$ and $\left(e_{3 m_{2}}^{6}\right) w=e_{3 n_{3}}^{2 *} \cdot p \in E_{27}^{2}$ is the only element such that $\left(e_{3 m_{1}+1}^{2}\right) p=e_{3 m_{1}}^{2}=e_{3\left(\Delta_{2}-m_{1}\right)+1}^{6 *}$ and

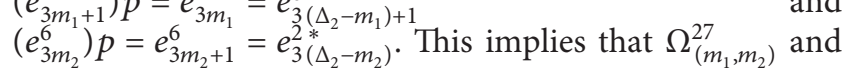




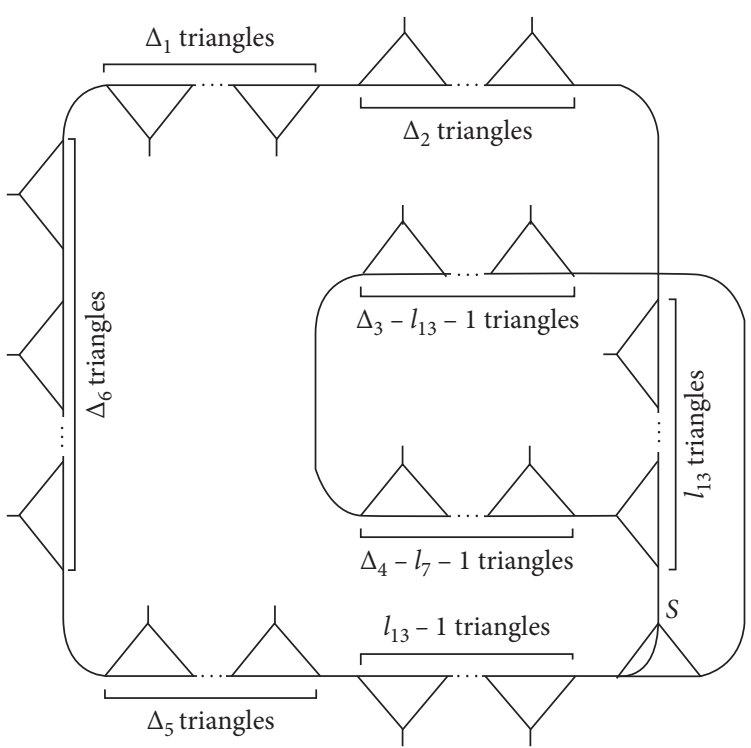

FIGURE 39: Homomorphic images $\Omega_{\left(l_{13}, l_{7}\right)}^{26}$.

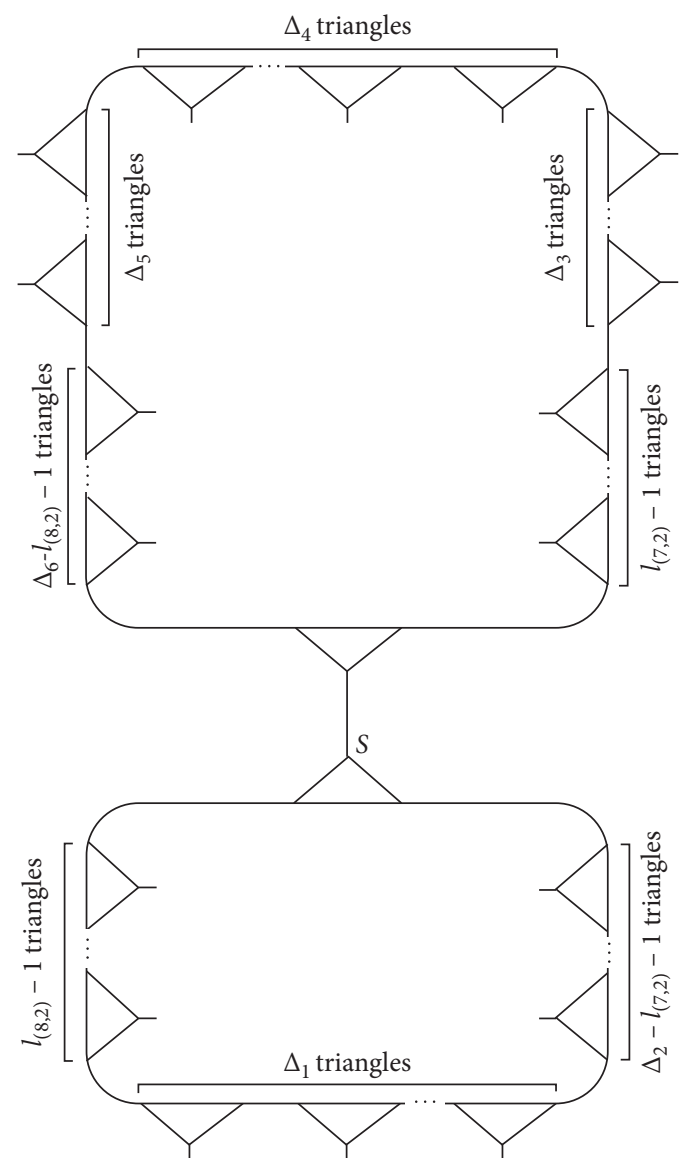

FIGURE 40: Homomorphic images $\Omega_{\left(l_{(7,2)}, l_{(8,2)}\right)}^{27}$. 


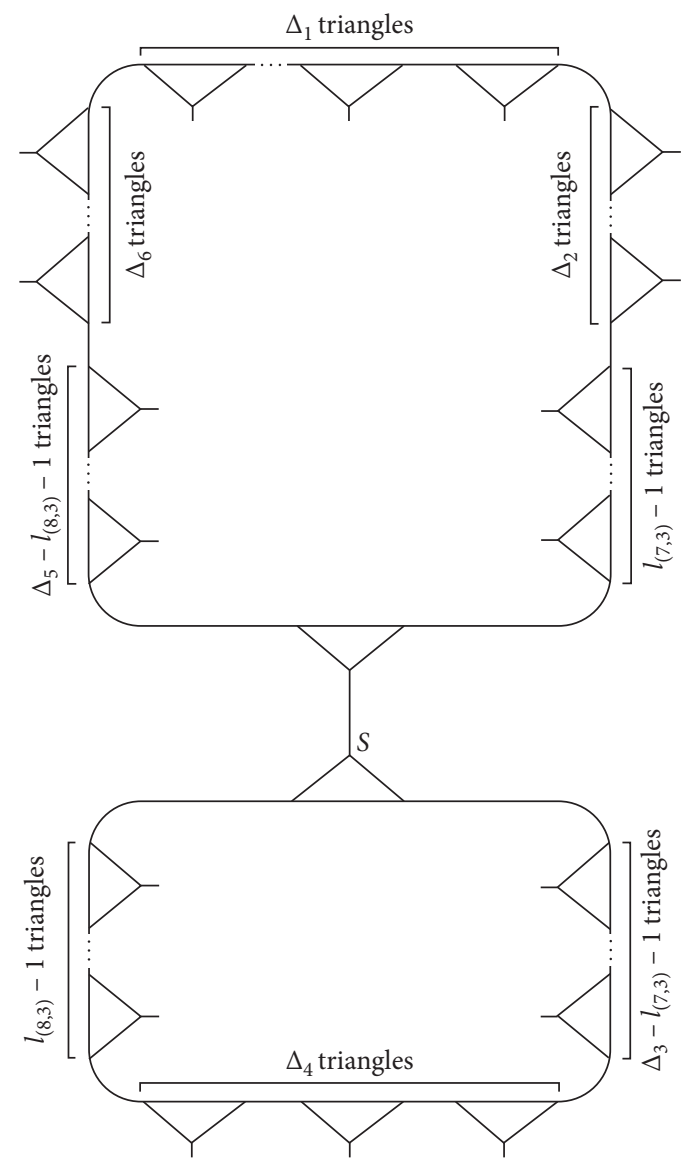

FIGURE 41: Homomorphic images $\Omega_{\left(l_{(7,3)}, l_{(8,3)}\right)}^{27}$.

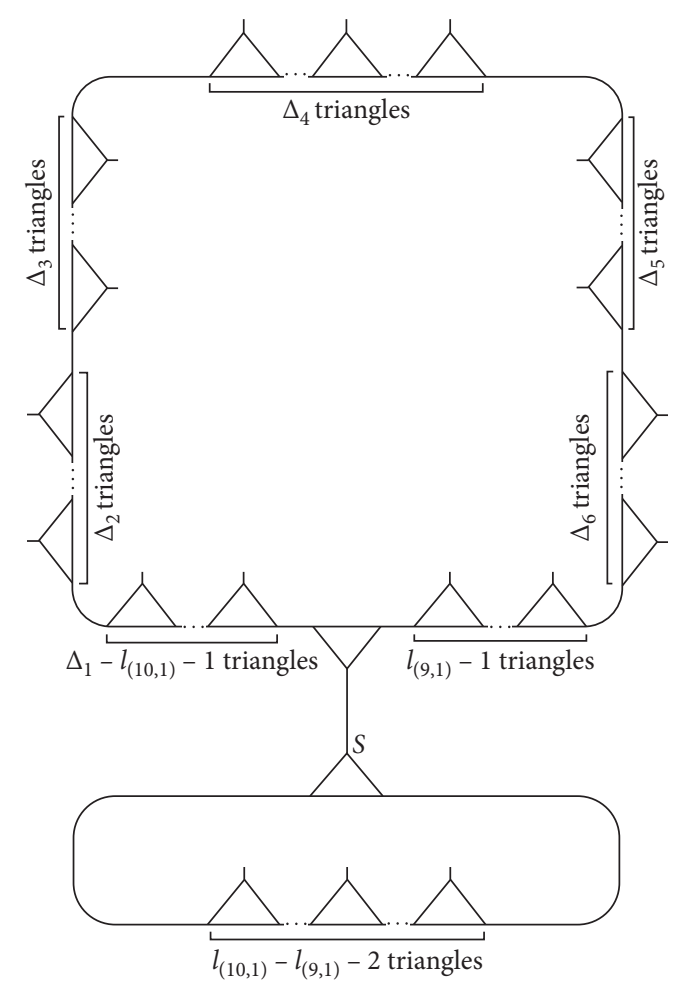

FIGURE 42: Homomorphic images $\Omega_{\left(l_{(9,1)}, l_{(10,1)}\right.}^{28}$. 
$\Omega_{\left(n_{1}, n_{2}\right)}^{27}$ are the mirror images of each other if and only if $n_{1}=\Delta_{2}-m_{2}$ and $n_{2}=\Delta_{2}-m_{1}$, that is, by contracting $e_{3 m_{1}+1}^{2}$ and $e_{3 m_{2}}^{6}$ to produce $\Omega_{\left(m_{1}, m_{2}\right)}^{27}, e_{3 n_{1}+1}^{2 *}$ and $e_{3 n_{2}}^{6 *}$ are also contracted. For a fix $m_{1} \in l_{(7,2)}$ and for all $m_{2} \in l_{(8,2)} \backslash\left\{\Delta_{2}-m_{1}\right\}$, $\Omega_{\left(\Delta_{2}-m_{2}, \Delta_{2}-m_{1}\right)}^{22} \notin H_{27}^{2}$, implying that $\Omega_{\left(m_{1}, m_{2}\right)}^{27}$ and $\Omega_{\left(n_{1}, n_{2}\right)}^{27}$ are not the mirror images of each other. This implies that all the homomorphic images in $H_{27}^{2}$ are distinct. Thus, $H_{27}^{2}=\left\{\Omega_{\left(l_{(7,2)}, l_{(8,2)}\right)}{ }^{27} ; l_{(7,2)}=1,2, \ldots, \Delta_{2}-1 ; l_{(8,2)}=1\right.$, $\left.2, \ldots, \Delta_{2}-l_{(7,2)}\right\}$ implies

$$
\left|H_{27}^{2}\right|=\frac{1}{2} \Delta_{2}\left(\Delta_{2}-1\right) \text {. }
$$

Let $\Omega_{\left(m_{1}, m_{2}\right)}^{27}$ be the mirror image of itself, then from Figure 40 , we have $m_{1}-1=\Delta_{2}-m_{2}-1$ and $m_{2}-1=\Delta_{2}-m_{1}-1$, implying $m_{2}=\Delta_{2}-m_{1}$. Now, $\forall m_{1} \in l_{(7,2)}, \Delta_{2}-m_{1} \in l_{(8,2)}$, implying that $\Omega_{\left(m_{1}, \Delta_{2}-m_{1}\right)}^{27} \in H_{27}^{2}$ is the mirror image of itself. So, out of $(1 / 2) \Delta_{2}\left(\Delta_{2}-1\right)$. homomorphic images in $H_{27}^{2}, \Delta_{2}-1$ are the mirror images of itself. Hence, there are

$$
2 \times\left|E_{27}^{2}\right| \times\left[\left|H_{27}^{2}\right|-\left(\Delta_{2}-1\right)\right]+\left|E_{27}^{2}\right| \times\left(\Delta_{2}-1\right)=6\left(\Delta_{2}-1\right)^{2}
$$

pairs of contracting vertices to produce all the homomorphic images in $H_{27}^{2}$. We can prove this lemma for $k_{2}=3$ (Figure 41) in similar way as that for $k_{2}=2$.

$$
\text { Let } k_{4}=\{1,4\} \text { and } \eta_{\left(4, k_{4}\right)}=\left\{\begin{array}{ll}
0 & \text { if } \Delta_{k_{4}}=0(\bmod 2) \\
1 & \text { otherwise }
\end{array} .\right.
$$

Lemma 28. If we contract the vertices $e_{3 l_{\left(7 k_{4}\right)+1}^{k_{4}}}: l_{\left(9, k_{4}\right)}=$ $1,2, \ldots, \quad\left(\left(\Delta_{k_{4}}-2-\eta_{\left(4, k_{4}\right)}\right) / 2\right)$, with the vertices $e_{3 l_{\left(10, k_{4}\right)}}^{k_{4}}: l_{\left(10, k_{4}\right)}=l_{\left(9, k_{4}\right)}+1, l_{\left(9, k_{4}\right)}+2, \ldots, \Delta_{k_{4}}-l_{\left(9, k_{4}\right)}$, in the circuit $\Delta$, then for each $k_{4}$, there arise $(1 / 4)\left(\Delta_{k_{4}}^{2^{-}}-2 \Delta_{k_{4}}+\right.$ $\left.\eta_{\left(4, k_{4}\right)}\right)$ distinct homomorphic images of $\Delta$ and 6 pairs of contracted vertices create each homomorphic image. Moreover, the number of total pairs of contracted vertices to generate all $(1 / 4)\left(\Delta_{k_{4}}^{2}-2 \Delta_{k_{4}}+\eta_{\left(4, k_{4}\right)}\right)$ and their mirror homomorphic images of $\Delta$ is $3\left(\Delta_{k_{4}}^{2}-3 \Delta_{k_{4}}+2\right)$.

Proof. For $\quad k_{4}=1, \quad$ let $\quad H_{28}^{1}=\left\{\Omega_{\left(l_{(9,1)}, l_{(10,1)}\right)}\right.$
${ }^{28} ; l_{(9,1)}=1,2, \ldots,\left(\left(\Delta_{1}-2-\eta_{(4,1)}\right) / 2\right) ; l_{(10,1)}=$ $\left.l_{(9,1)}+1, l_{(9,1)}+2, \ldots, \Delta_{1}-l_{(9,1)}\right\}$ (Figure 42) be the collection of homomorphic images of $\Delta$ acquired by the contraction of the vertices $e_{3 l_{(9,1)}+1}^{1}$ with the vertices $e_{3 l_{(10,1)}}^{1}$ in $\Delta$, where $e_{3 l_{(2,+1}}^{1}$ and $e_{3 l_{(1,1)}^{1}}^{1}$ are fixed by the words $(p q)^{l_{(9,1)}}\left(p q^{-1}\right)^{\Delta_{6}^{+1}}(p q)^{\Delta_{5}}\left(p q^{\left.-{ }^{-1}\right)^{1 \Delta_{4}}}(p q)^{\Delta_{3}}\left(p q^{-1}\right)^{\Delta_{2}}(p q)^{\Delta_{1}-l_{(9,1)}}\right.$ and $\left(p q^{-1}\right)^{\Delta}$ ${ }_{1}-l_{(10,1)}(p q)^{\Delta_{2}}\left(p q^{-1}\right)^{\Delta_{3}}(p q)^{\Delta_{4}}\left(p q^{-1}\right)^{\Delta_{5}}(p q)^{\Delta_{6}}\left(p q^{-1}\right)^{l_{(10,1)}}$. It is easy to verify that $P_{1}=(p q)^{l_{(9,1)}}\left(p q^{-1}\right)^{\Delta}$ ${ }_{6}(p q)^{\Delta_{5}}\left(p q^{-1}\right)^{\Delta_{4}}(p q)^{\Delta_{3}}\left(p q^{-1}\right)^{\Delta_{2}}(p q)^{\Delta_{1}-l_{(10,1)}} p$ and $P_{2}=q(p q)^{l_{(10,1)}-l_{(9,1)}-1}$ are the possible paths between $e_{3 l_{(9,1)}+1}^{1}$ and $e_{3 l}^{1}$ (Figure 4). This implies that the homomorphic image $\Omega_{\left(l_{(9,1)}, l_{(10,1)}\right)}^{28}$ has a vertex $s$ fixed by $P_{1}$ and $P_{2}$. Thus,

$$
E_{28}^{1}=\left\{e, q, q^{-1}, p, p q, p q^{-1}\right\}
$$

is the family of elements in $\operatorname{PSL}(2, \mathbb{Z})$ such that $\forall w \in E_{28}^{1}, \Delta$ contains the vertices $\left(e_{3 l_{\left(9_{1}\right)+1}}^{1}\right) w$ and $\left(e_{3 l_{(10,1)}}^{1}\right) w$. This gives that the cardinality of $E_{28}^{1^{1}}$, that is, 6 is the number of contracted pairs of vertices to produce each homomorphic image $\Omega_{\left(l_{(9,1)}, l_{(10,8)}\right)}^{28}$ (Theorem 2).

Suppose $\Omega_{\left(m_{1}, m_{2}\right)}^{28}$ and $\Omega_{\left(n_{1}, n_{2}\right)}^{28}$ are the same. This concludes that $\Omega_{\left(m_{1}, m_{2}\right)}^{28}$ is procurable also by contracting $e_{3 n_{1}+1}^{1}$ and $e_{3 n_{2}}^{1}$, implying that $e_{3 n_{1}+1}^{1} \leftrightarrow e_{3 n_{2}}^{1}$ is one of the pairs of contracted vertices for $\Omega_{\left(m_{1}, m_{2}\right)}^{28^{1}}$. Then there must exist an element $w \in E_{28}^{1} \quad$ such that $\left(e_{3 m_{1}+1}^{1}\right) w=e_{3 n_{1}+1}^{1}$ and $\left(e_{3 m_{2}}^{1}\right) w=e_{3 n_{2}}^{1} \cdot p q, p q^{-1} \in E_{28}^{1}$ are the only element such that $\left(e_{3 m_{1}+1}^{1}\right) p q=e_{3\left(m_{1}-1\right)+1}^{1}$ and $\left(e_{3 m_{2}}^{1}\right) p q^{-1}=e_{3\left(m_{2}+1\right)}^{1}$ but $\left(e_{3 m_{2}}^{1}\right) p q \neq e_{3 n_{2}}^{1}$ and $\left(e_{3 m_{1}+1}^{1}\right) p q^{-1} \neq e_{3 n_{1}+1}^{1} \cdot \Omega_{\left(m_{1} m_{2}\right)}^{28}$ and $\Omega_{\left(n_{1}, n_{2}\right)}^{28}$ are not the same, that is, by contracting $e_{3 m_{1}+1}^{1}$ and $e_{3 m_{2}}^{1}$ to produce $\Omega_{\left(m_{1}, m_{2}\right)}^{28}, e_{3 n_{1}+1}^{1}$ and $e_{3 n}^{1}$ are not contracted. Now, if $\Omega_{\left(m_{1}, m_{2}\right)}^{28}$ and $\Omega_{\left(n_{1}, n_{2}\right)}^{28 *}$ are same, then there must exist an element $w \in E_{28}^{1}$ such that $\left(e_{3 m_{1}+1}^{1}\right) w=e_{3 n_{1}+1}^{1 *}$ and $\left(e_{3 m_{2}}^{1}\right) w=e_{3 n_{1}}^{1 *} \cdot p \in E_{28}^{1}$ is the only element such that $\left(e_{3 m_{1}+1}^{1}\right) p=e_{3 m_{1}}^{1}=e_{3\left(\Delta_{1}-m_{1}\right)+1}^{1 *} \quad$ and $\quad\left(e_{3 m_{2}}^{1}\right) p=e_{3 m_{2}+1}^{1}=$ $e_{3\left(\Delta_{1}-m_{2}\right)}^{1 *}$ This implies that $\Omega_{\left(m_{1}, m_{2}\right)}^{28}$ and $\Omega_{\left(n_{1}, n_{2}\right)}^{28}$ are the mirror images of each other if and only if $n_{1}=\Delta_{1}-m_{1}$ and $n_{2}=\Delta_{1}-m_{2}$, that is, by contracting $e_{3 m_{1}+1}^{1}$ and $e_{3 m_{2}}^{1}$ to produce $\Omega_{\left(m_{1}, m_{2}\right)}^{28}, e_{3 n_{1}+1}^{1 *}$ and $e_{3 n_{2}}^{1 *}$ are also contracted. For all $m_{1} \in l_{(9,1)}, \quad \Delta_{1}-m_{1}>\left(\left(\Delta_{1}-2-\eta_{(4,1)}\right) / 2\right)$, implying that $\Omega_{\left(n_{1}, n_{2}\right)}^{28}=\Omega_{\left(\Delta_{2}-m_{2}, \Delta_{2}-m_{1}\right)}^{28} \notin H_{28}^{1}$ and that $\Omega_{\left(m_{1}, m_{2}\right)}^{28}$ and $\Omega_{\left(n_{1}, n_{2}\right)}^{28}$ are not the mirror images of each other. This implies that all the homomorphic images in $H_{28}^{1}$ are distinct. Thus,

$$
H_{28}^{1}=\left\{\Omega_{\left(l_{(9,1)}, l_{(10,1)}\right)}^{28} ; l_{(9,1)}=1,2, \ldots, \frac{\Delta_{1}-2-\eta_{(4,1)}}{2} ; l_{(10,1)}=l_{(9,1)}+1, l_{(9,1)}+2, \ldots, \Delta_{1}-l_{(9,1)}\right\}
$$

implies

$$
\begin{aligned}
\left|H_{28}^{1}\right| & =\left(\Delta_{1}-2\right)+\left(\Delta_{2}-4\right)+\left(\Delta_{2}-6\right)+\cdots+\left(4-\eta_{(4,1)}\right) \\
& +\left(2-\eta_{(4,1)}\right), \\
& =\frac{1}{4}\left(\Delta_{1}^{2}-2 \Delta_{1}+\eta_{(4,1)}\right) .
\end{aligned}
$$

Let $\Omega_{\left(m_{1}, m_{2}\right)}^{28}$ be the mirror image of itself, then from Figure 42 , we have $m_{1}-1=\Delta_{1}-m_{2}-1$, implying $m_{2}=\Delta_{1}-m_{1}$. Now, $\forall m_{1} \in l_{(9,1)}, \Delta_{1}-m_{1} \in l_{(10,1)}$, implying that $\Omega_{\left(m_{1}, \Delta_{1}-m_{1}\right)}^{28} \in H_{28}^{1}$ is the mirror image of itself. So, out of $(1 / 4)\left(\Delta_{1}^{2}-2 \Delta_{1}+\eta_{(4,1)}\right)$ homomorphic images in $H_{28}^{1}$, $\left(\left(\Delta_{1}-2-\eta_{(4,1)}\right) / 2\right)$ are the mirror images of itself. Hence, there are 


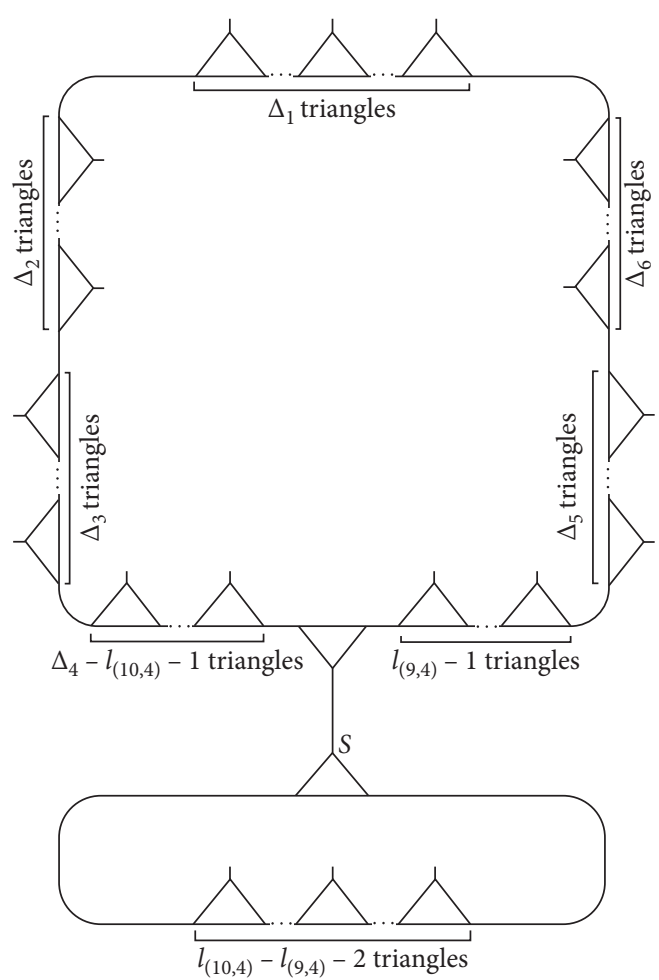

FIGURE 43: Homomorphic images $\Omega_{\left(l_{(9,4)}, l_{(10,4)}\right.}^{28}$.

$$
\begin{aligned}
2 & \times\left|E_{28}^{2}\right| \times\left[\left|H_{28}^{2}\right|-\frac{\Delta_{1}-2-\eta_{(4,1)}}{2}\right]+\left|E_{28}^{2}\right| \times \frac{\Delta_{1}-2-\eta_{(4,1)}}{2} \\
& =3\left(\Delta_{1}^{2}-3 \Delta_{1}+2\right)
\end{aligned}
$$

pairs of contracting vertices to produce all the homomorphic images in $H_{28}^{1}$. We can prove this lemma for $k_{4}=4$ (Figure 43) in similar way as that for $k_{4}=1$.

Lemma 29. If we contract the vertices $e_{3 l_{\left(11, k_{2}\right)}+1}^{k_{2}}: l_{\left(11, k_{2}\right)}=$ $1,2, \ldots, \Delta_{k_{2}}-1, \quad$ with the $l_{\left(11, k_{2}\right)}$ vertices $e_{3 l_{\left(12, k_{2}\right)}^{k_{2}}:} l_{\left(12, k_{2}\right)}=1,2, \ldots, \Delta_{k_{2}}-l_{\left(11, k_{2}\right)}$, in the circuit $\Delta$, then for each $k_{2}$, there arise $(1 / 6)\left(\Delta_{k_{2}}-1\right)\left(\Delta_{k_{2}}-2\right)$ distinct homomorphic images of $\Delta$ and 6 pairs of contracted vertices create each homomorphic image. Moreover, the number of total pairs of contracted vertices to generate all $(1 / 2)\left(\Delta_{k_{2}}-\right.$ $1)\left(\Delta_{k_{2}}-2\right)$ and their mirror homomorphic images of $\Delta$ is $6\left(\Delta_{k_{2}}-1\right)\left(\Delta_{k_{2}}-2\right)$.

For a fix value of $k_{2}$, let $H_{29}^{k_{2}}=\left\{\Omega_{\left(l_{\left(11, k_{2}\right)}, l\right.}\right.$ $\left.\left(12, k_{2}\right)\right)^{29} ; l_{\left(11, k_{2}\right)}=1,2, \ldots, \Delta_{k_{2}}-1 ; l_{\left(12, k_{2}\right)}=1,2, \ldots, \Delta_{k_{2}}$, $\left.l_{\left(11, k_{2}\right)}\right\}$ be the collection of homomorphic images of $\Delta$ acquired by the contraction of the vertices $e_{3 l_{\left(11, k_{2}\right)^{+1}}}^{k_{2}}$ with the vertices $e_{3 l_{\left(12, k_{2}\right)}^{k_{2}}}$ in $\Delta$. Figures 44 and 45 present $H_{29}^{k_{2}}$ graphically. From all the homomorphic images presented in these figures, it is not intricated to check that no one is the mirror image of itself. This lemma can be proved by using the same procedure as that for Lemma 23.

$$
\text { Letk }_{4}=\{1,4\} \text { and } \eta_{\left(4, k_{4}\right)}=\left\{\begin{array}{ll}
0 & \text { if } \Delta_{k_{4}}=0(\bmod 2) \\
1 & \text { otherwise }
\end{array} .\right.
$$

Lemma 30. If we contract the vertices $e_{3 l_{\left(13, k k_{2}+1\right.}^{k_{4}}}: l_{\left(13, k_{4}\right)}=$

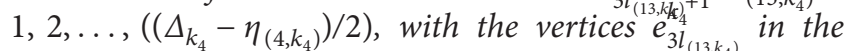
circuit $\Delta$, then for each $k_{4}$, there arise $(1 / 2)\left(\Delta_{k_{4}}-\eta_{\left(4, k_{4}\right)}\right)$ distinct homomorphic images of $\Delta$ and 3 pairs of contracted vertices create each homomorphic image. Moreover, the number of total pairs of contracted vertices to generate all $(1 / 2)\left(\Delta_{k_{4}}-\eta_{\left(4, k_{4}\right)}\right)$ and their mirror homomorphic images of $\Delta$ is $3\left(\Delta_{k_{4}}-1\right)$.

$H_{30}^{k_{4}}=\left\{\Omega_{\left(l_{\left(13, k_{4}\right)}, l_{\left(13, k_{4}\right)}\right)}^{\text {For }} ; l_{\left(13, k_{4}\right)}=1,2, \ldots,\left(\left(\Delta_{k_{4}}-\eta_{\left(4, k_{4}\right)}\right) / 2\right)\right\}^{\text {let }}$ be the collection of homomorphic images of $\Delta$ acquired by the contraction of the vertices $e_{3 l_{\left(13, k_{4}\right)^{+1}}}^{k_{4}}$ with the vertices $e_{3 l_{\left(13, k_{4}\right)}}^{k_{4}}$ in $\Delta$. Figures 46 and 47 present $H_{30}^{k_{4}}$ graphically. From all the homomorphic images presented in these figures, it is not intricated to check that if $\Delta_{k_{2}}=0(\bmod 2)$, then $\Omega_{\left(\left(\Delta_{k_{2}} / 2\right),\left(\Delta_{k_{2}} / 2\right)\right)}^{30}$ is the only homomorphic image which is the mirror image of itself; otherwise, no one is the mirror image of itself.

This lemma can be proved by using the same procedure as that for Lemma 28.

Lemma 31. If we contract the vertices $e_{3 l_{\left(11, k_{2}\right)+1}}^{k_{2}}: l_{\left(11, k_{2}\right)}=$ $1,2, \ldots, \Delta_{k_{2}}-1$, with the vertices $e_{3 l_{\left(11, k_{2}\right.}}^{k_{2}}$ in the circuit $\Delta$, then for each $k_{2}$, there arise $\Delta_{k_{2}}-1$ distinct homomorphic images of $\Delta$ and 3 pairs of contracted vertices create each homomorphic image. Moreover, the number of total pairs of contracted vertices to generate all $\Delta_{k_{2}}-1$ and their mirror homomorphic images of $\Delta$ is $6\left(\Delta_{k_{2}}-1\right)$.

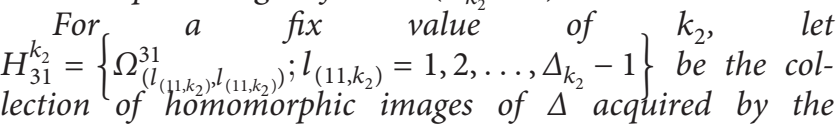




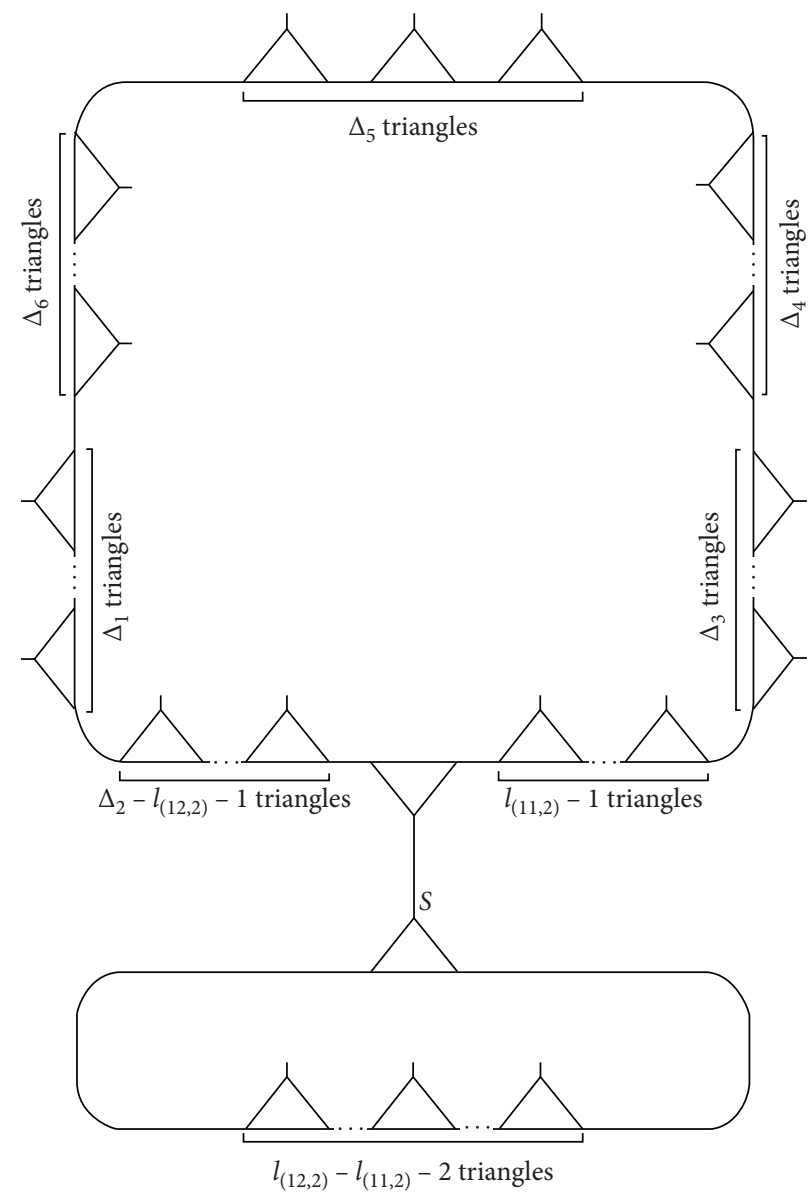

FIGURE 44: Homomorphic images $\Omega_{\left(l_{(1,2)}, l_{(12,2)}\right.}^{29}$.

contraction of the vertices $e_{3 l_{(11 k)+1}}^{k_{2}}$ with the vertices $e_{3 l_{(11 k)}}^{k_{2}}$ in $\Delta$. Figures 48 and 49 present $H_{31}^{k_{2}}$ graphically. From all ${ }^{\left(11 k^{2}\right)}$ the homomorphic images presented in these figures, it is not intricated to check that no one is the mirror image of itself. This lemma can be proved by using same procedure as that for Lemma 23.

Let $k_{5}=\{2,4\}$.

Lemma 32. For each $k_{5}$, there are $6\left(\Delta_{k_{5}}+\Delta_{k_{5}+1}+2\right)$ pairs of contracting vertices to produce the homomorphic image of $\Delta$ by contracting the vertex $e_{1}^{1}$ with the vertex $e_{1}^{k_{5}}$.
For $k_{5}=2$, let $\Omega_{2}^{32}$ be the homomorphic image of $\Delta$ acquired by contracting the vertex $e_{1}^{1}$ with the vertex $e_{1}^{2}$ in $\Delta$, where $e_{1}^{1}$ and $e_{1}^{2}$ are fixed by the words $\left(p q^{-1}\right)^{\Delta_{6}}(p q)^{\Delta_{5}}\left(p q^{-1}\right)^{\Delta_{4}}(p q)^{\Delta_{3}}\left(p q^{-1}\right)^{\Delta_{2}}(p q)^{\Delta_{1}}$ and $\left(p q^{-1}\right)^{\Delta_{3}}(p q)^{\Delta_{4}}\left(p q^{-1}\right)^{\Delta_{5}}(p q)^{\Delta_{6}}\left(p q^{-1}\right)^{\Delta_{1}}(p q)^{\Delta_{2}}$. It is easy to verify that $P_{1}=\left(p q^{-1}\right)^{\Delta_{6}}(p q)^{\Delta_{5}}\left(p q^{-1}\right)^{\Delta_{4}}(p q)^{\Delta_{3}} p$ and $P_{2}=$ $q^{-1}\left(p q^{-1}\right)^{\Delta_{2}-1}(p q)^{\Delta_{1}}$ are the possible paths between $e_{1}^{1}$ and $e_{1}^{2}$ (Figure 4). This implies that the homomorphic image $\Omega_{2}^{32}$ has a vertex $s$ fixed by $P_{1}$ and $P_{2}$. Thus,

$$
E_{2}^{32}=\left\{\begin{array}{c}
e, q, q^{-1}, p, p q,\left(p q^{-1}\right)^{1},\left(p q^{-1}\right)^{1} p,\left(p q^{-1}\right)^{1} p q,\left(p q^{-1}\right)^{2}, \ldots,\left(p q^{-1}\right)^{\Delta_{3}} p,\left(p q^{-1}\right)^{\Delta_{3}} p q, \\
\left(p q^{-1}\right)^{\Delta_{3}+1},\left(q^{-1} p\right)^{1},\left(q^{-1} p\right)^{1} q,\left(q^{-1} p\right)^{1} q^{-1}, \ldots,\left(q^{-1} p\right)^{\Delta_{2}},\left(q^{-1} p\right)^{\Delta_{2}} q,\left(q^{-1} p\right)^{\Delta_{2}} q^{-1}
\end{array}\right\}
$$

is the family of elements in $\operatorname{PSL}(2, \mathbb{Z})$ such that $\forall w \in E_{2}^{32}, \Delta$ contains the vertices $\left(e_{1}^{1}\right) w$ and $\left(e_{2}^{1}\right) w$. This gives that the cardinality of $E_{2}^{32}$, that is, $3\left(\Delta_{2}+\Delta_{3}+2\right)$ is the number of contracted pairs of vertices to produce the homomorphic image $\Omega_{2}^{32}$ (Theorem 2). From Figure 50, it is not intricated to check that $\Omega_{2}^{32}$ and its mirror image $\Omega_{2}^{32 *}$ are not the same. Hence, there are

$$
2 \times\left|E_{32}^{2}\right|=3\left(\Delta_{2}+\Delta_{3}+2\right),
$$




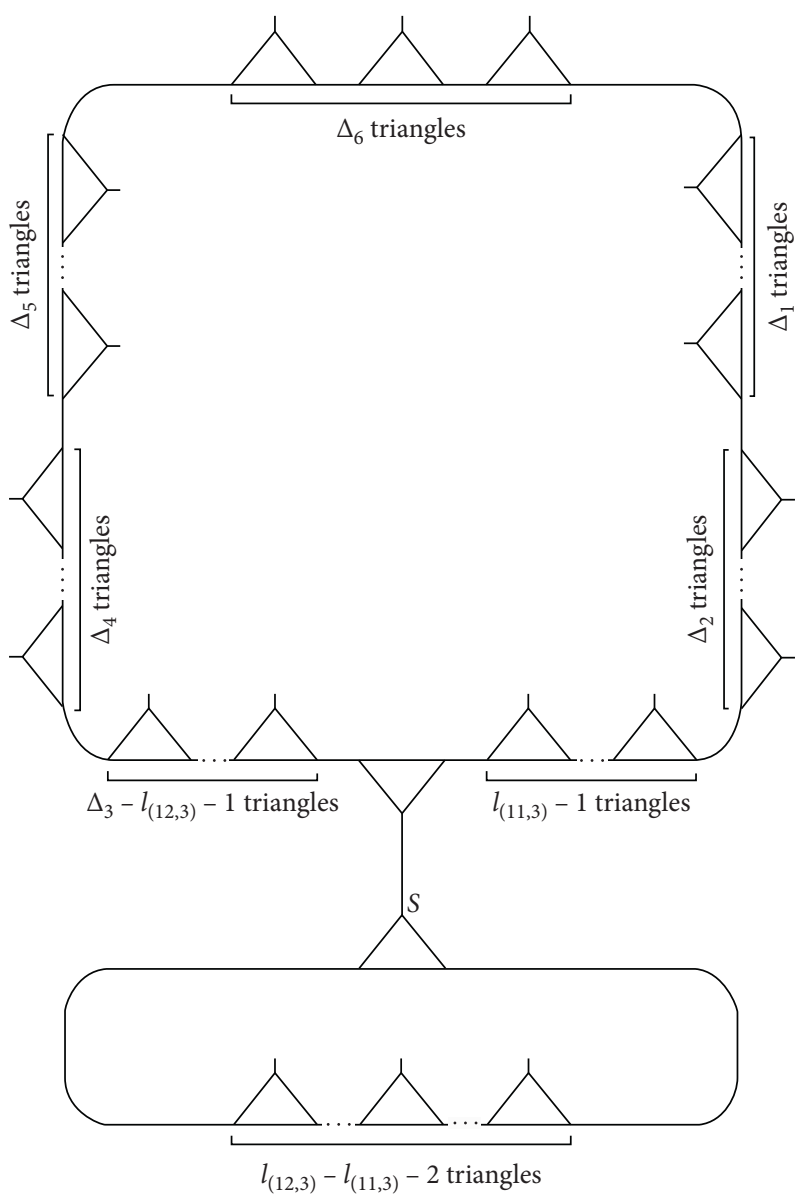

FIgURE 45: Homomorphic images $\Omega_{\left(l_{(11,3)}, l_{(12,3)}\right.}^{29}$.

pairs of contracting vertices to produce the homomorphic images $\Omega_{2}^{32}$.

We can prove this lemma for $k_{5}=4$ (Figure 51) in similar way as that for $k_{5}=2$.

Lemma 33. For each $k_{3}$, there are $6\left(\Delta_{4}+\Delta_{5}+2\right)$ pairs of contracting vertices to produce the homomorphic image of $\Delta$ by contracting the vertex $e_{1}^{5}$ with the vertex $e_{1}^{k_{3}}$.

For a fix value of $k_{3}$, let $\Omega_{k_{3}}^{33}$ be the homomorphic image of $\Delta$ acquired by the contraction of the vertex $e_{1}^{5}$ with the vertex $e_{1}^{k_{3}}$ in $\Delta$. Figures 52 and 53 present $\Omega_{k_{3}}^{33}$ graphically. From these figures, it is not intricated to check that $\Omega_{k_{3}}^{33}$ is not the mirror image of itself.

This lemma can be proved by using the same procedure as that for Lemma 32.

$$
\text { Let } k_{6}=\{1,2,5\} \text { and } \overline{k_{6}}= \begin{cases}3 & \text { if } k_{6}=2 \text {, . } \\ 4 & \text { if } k_{6}=5 .\end{cases}
$$

Lemma 34. For each $k_{6}$, there are $6\left(\Delta_{\bar{k}}+1\right)$ pairs of contracting vertices to produce the homomorphic image of $\Delta$ by contracting the vertex $e_{1}^{k_{6}}$ with the vertex $e_{1}^{k_{6}}$.

For a fix value of $k_{6}$, let $\Omega_{k_{6}}^{34}$ be the homomorphic image of $\Delta$ acquired by the contraction of the vertex $e_{1}^{k_{6}}$ with the vertex $e_{1}^{k_{6}}$ in $\Delta$. Figures 54, 55, and 56 present $\Omega_{k_{6}}^{34}$ graphically. From these figures, it is not intricated to check that $\Omega_{k_{6}}^{34}$ is not the mirror image of itself. This lemma can be proved by using the same procedure as that for Lemma 32.

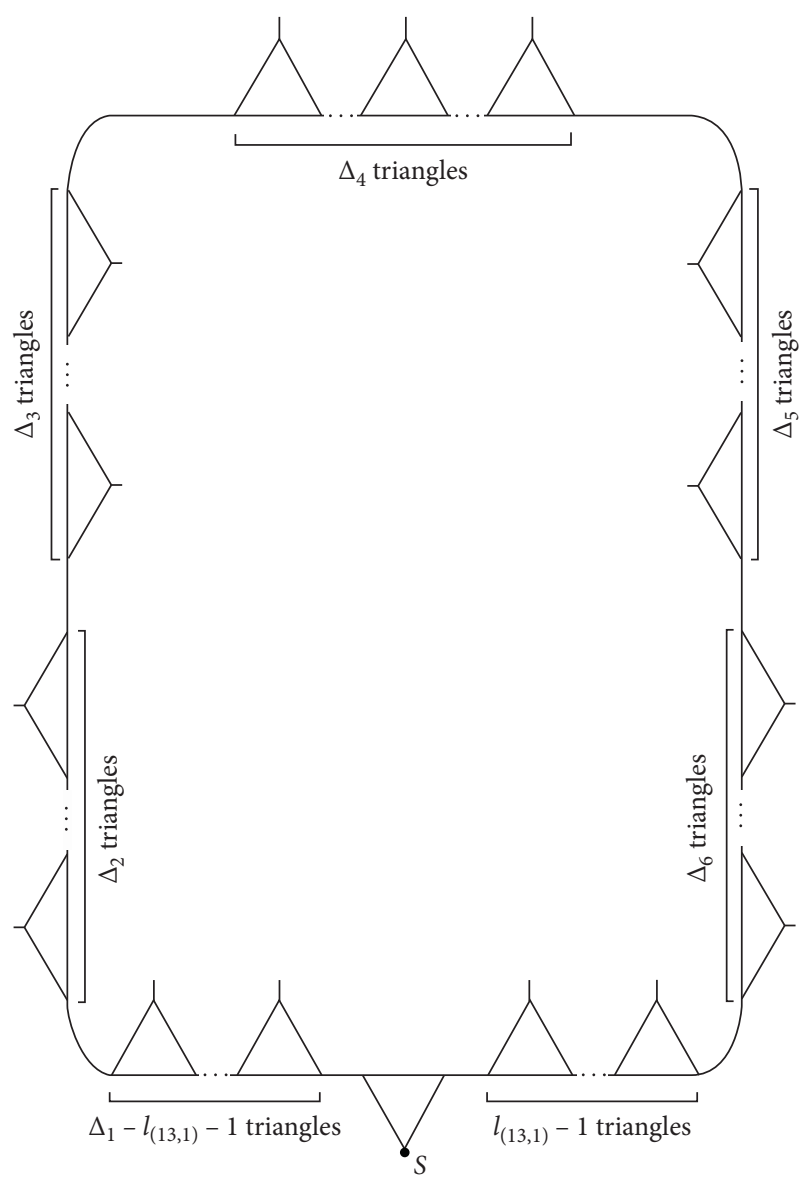

FIGURE 46: Homomorphic images $\Omega_{\left(l_{(13,1)}, l_{(13,1)}\right)}^{30}$.

Lemma 35. For each $k_{3}$, there are $3\left(\Delta_{k_{3}+1}+2 \Delta_{k_{3}+2}+2\right)$ pairs of contracting vertices to produce the homomorphic image of $\Delta$ by contracting the vertex $e_{1}^{k_{3}}$ with the vertex $e_{1}^{k_{3}+2}$.

For a fix value of $k_{3}$, let $\Omega_{k_{3}}^{35}$ be the homomorphic image of $\Delta$ acquired by the contraction of the vertex $e_{1}^{k_{3}}$ with the vertex $e_{1}^{k_{3}+2}$ in $\Delta$. Figures 57(a), 57(b), and 57(c) present $\Omega_{1}^{35}$ and Figure 58 presents $\Omega_{2}^{35}$ graphically. From these figures, it is not intricated to check that $\Omega_{k_{3}}^{35}$ is the mirror image of itself. This lemma can be proved by using the same procedure as that for Lemma 32.

Let $V(\Delta \times \Delta)=\{(a, b) ; a, b \in V(\Delta)$ and $a \neq b\}$, then

$|V(\Delta \times \Delta)|=\frac{1}{2}\left[9\left(\Delta_{1}+2 \Delta_{2}+2 \Delta_{3}+\Delta_{4}\right)-3\left(\Delta_{1}+2 \Delta_{2}+2 \Delta_{3}+\Delta_{4}\right)\right]$.

We are now able to prove our primary outcome. Let

$$
\eta= \begin{cases}4, & \text { if }\left(\Delta_{1}, \Delta_{4}\right)=(E, E), \\ 0, & \text { if }\left(\Delta_{1}, \Delta_{4}\right)=(O, O), \\ -1, & \text { otherwise. }\end{cases}
$$

where $E$ and $O$ stand for even and odd, respectively.

Theorem 5. There are $(1 / 4)\left[\left(\Delta_{1}+2 \Delta_{2}+2 \Delta_{3}+\Delta_{4}\right)^{2}+\right.$ $\left.4\left(\Delta_{1}+2 \Delta_{2}+2 \Delta_{3}+\Delta_{4}\right)+4 \Delta_{4}+\eta\right]$ number of distinct 


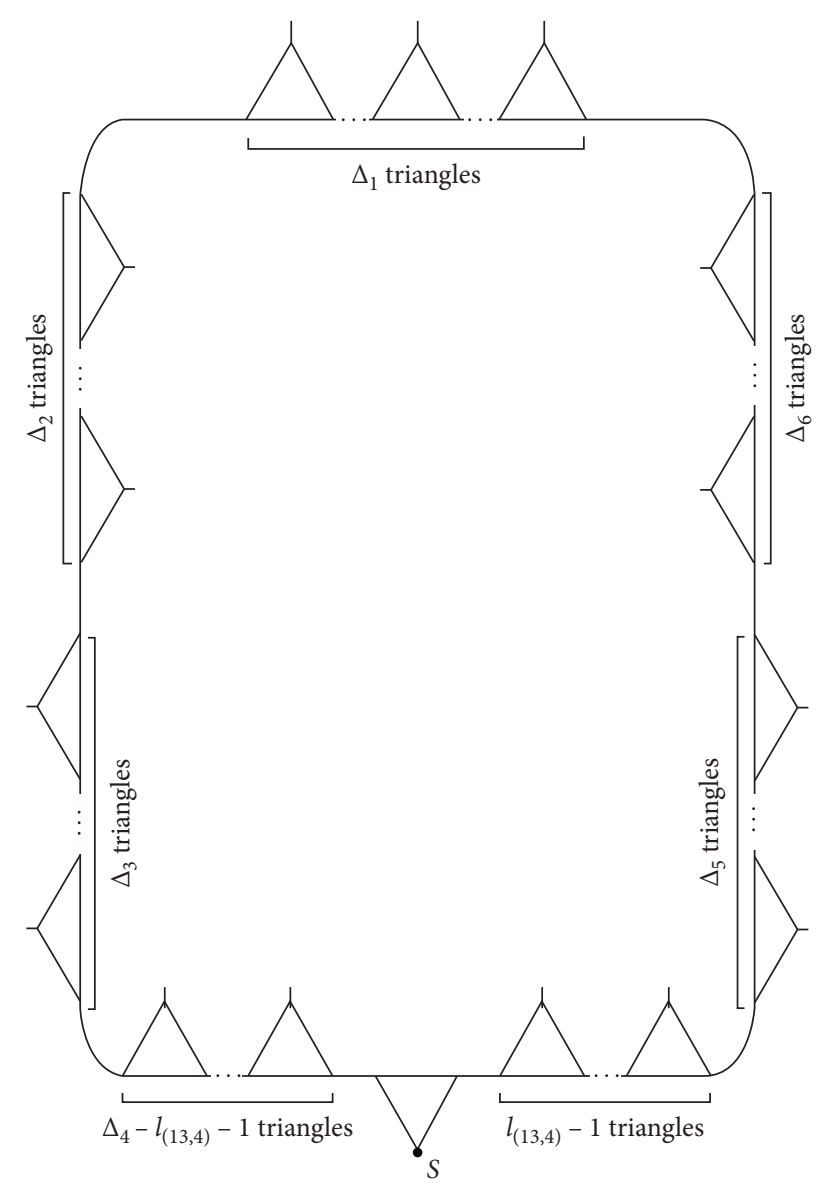

FIgURE 47: Homomorphic images $\Omega_{\left(l_{(13,4)}, l_{(13,4)}\right.}^{30}$.

homomorphic images acquired by the contraction of all pairs of vertices of the circuit $\Delta$.

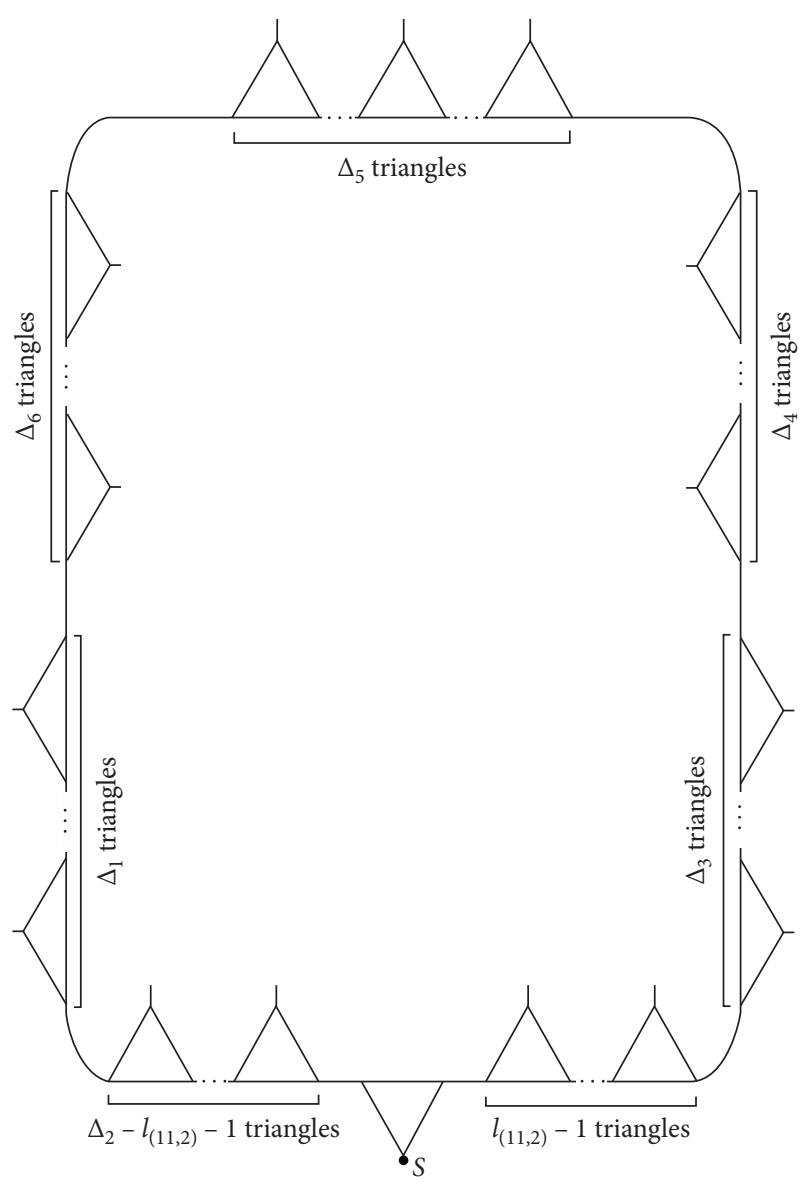

FIGURE 48: Homomorphic images $\Omega_{\left(l_{(1,2)}, l_{(11,2)}\right)}^{31}$.

Proof. Let us collect all the pairs of contracting vertices of $\Delta$ mentioned in Lemma 1 to Lemma 35 in the form of set $S$ as

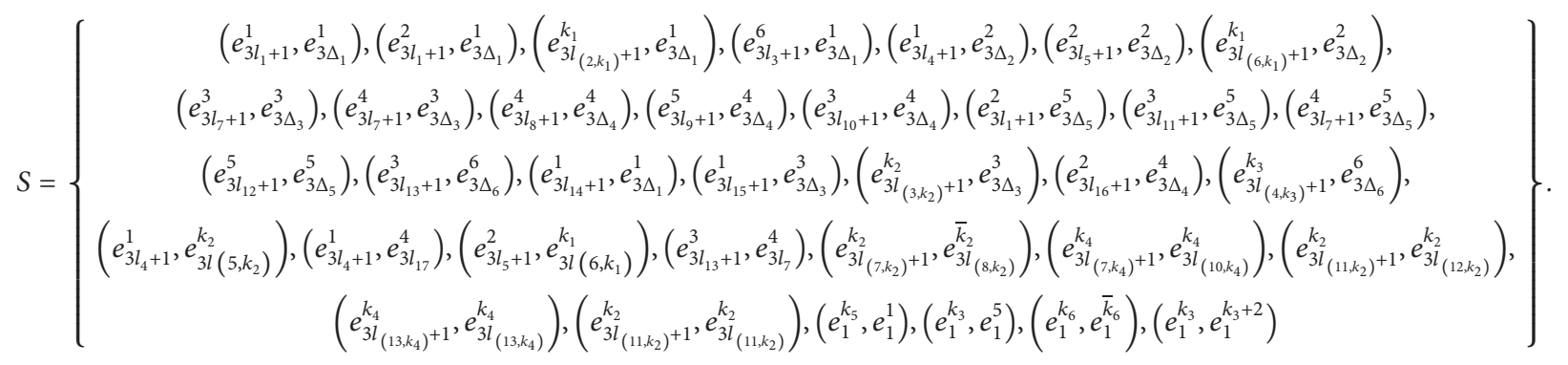

Let $H$ be the set of homomorphic images obtained by contracting each element of $\mathrm{S}$, then

$$
H=\left\{\begin{array}{c}
\Omega_{l_{1}}^{1}, \Omega_{l_{1}}^{2}, \Omega_{l_{\left(2, k_{1}\right)}}^{3}, \Omega_{l_{3}}^{4}, \Omega_{l_{4}}^{5}, \Omega_{l_{5}}^{6}, \Omega_{l_{\left(6, k_{1}\right)}}^{7}, \Omega_{l_{7}}^{8}, \Omega_{l_{7}}^{9}, \Omega_{l_{8}}^{10}, \Omega_{l_{9}}^{11}, \Omega_{l_{l_{0}}}^{12}, \Omega_{l_{1}}^{13}, \Omega_{l_{11}}^{14}, \Omega_{l_{7}}^{15}, \Omega_{l_{l_{2}}}^{16}, \Omega_{l_{13}}^{17}, \\
\Omega_{l_{l_{1}}}^{18}, \Omega_{l_{15}}^{19}, \Omega_{l_{\left(3, k_{2}\right)}}^{20}, \Omega_{l_{16}}^{21}, \Omega_{l_{\left(4, k_{3}\right)}}^{22}, \Omega_{\left(l_{4}, l_{\left(5, k_{2}\right)}\right)}^{23}, \Omega_{\left(l_{4}, l_{17}\right)}^{24}, \Omega_{\left(l_{5}, l_{\left(6, k_{1}\right)}\right)}^{25}, \Omega_{\left(l_{13}, l_{7}\right)}^{26}, \Omega_{\left(l_{\left(7, k_{2}\right)}, l_{\left(8, k_{2}\right)}\right)}^{27}, \\
\Omega_{\left(l_{\left(9, k_{4}\right)}, l_{\left(10, k_{4}\right)}\right)}^{28}, \Omega_{\left(l_{\left(11, k_{2}\right)}, l_{\left(12, k_{2}\right)}\right)}^{29}, \Omega_{\left(l_{\left(13, k_{4}\right)}, l_{\left(13, k_{4}\right)}\right)}^{30}, \Omega_{\left(l_{\left(11, k_{2}\right)}, l_{\left(1, k_{2}\right)}\right)}^{31}, \Omega_{k_{5}}^{32}, \Omega_{k_{3}}^{33}, \Omega_{k_{6}}^{34}, \Omega_{k_{3}}^{35}
\end{array}\right\} .
$$




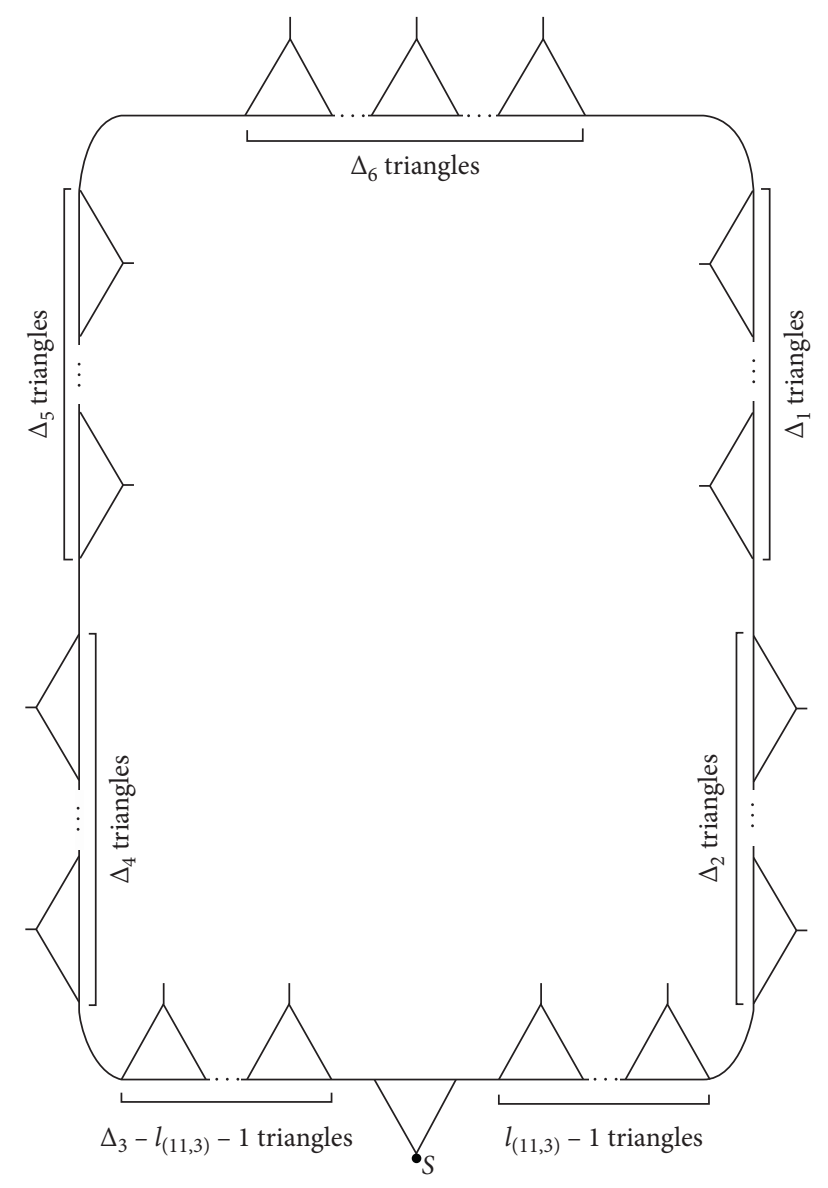

FIgURE 49: Homomorphic images $\Omega_{\left(l_{(1,3)}, l_{(11,3)}\right.}^{31}$.

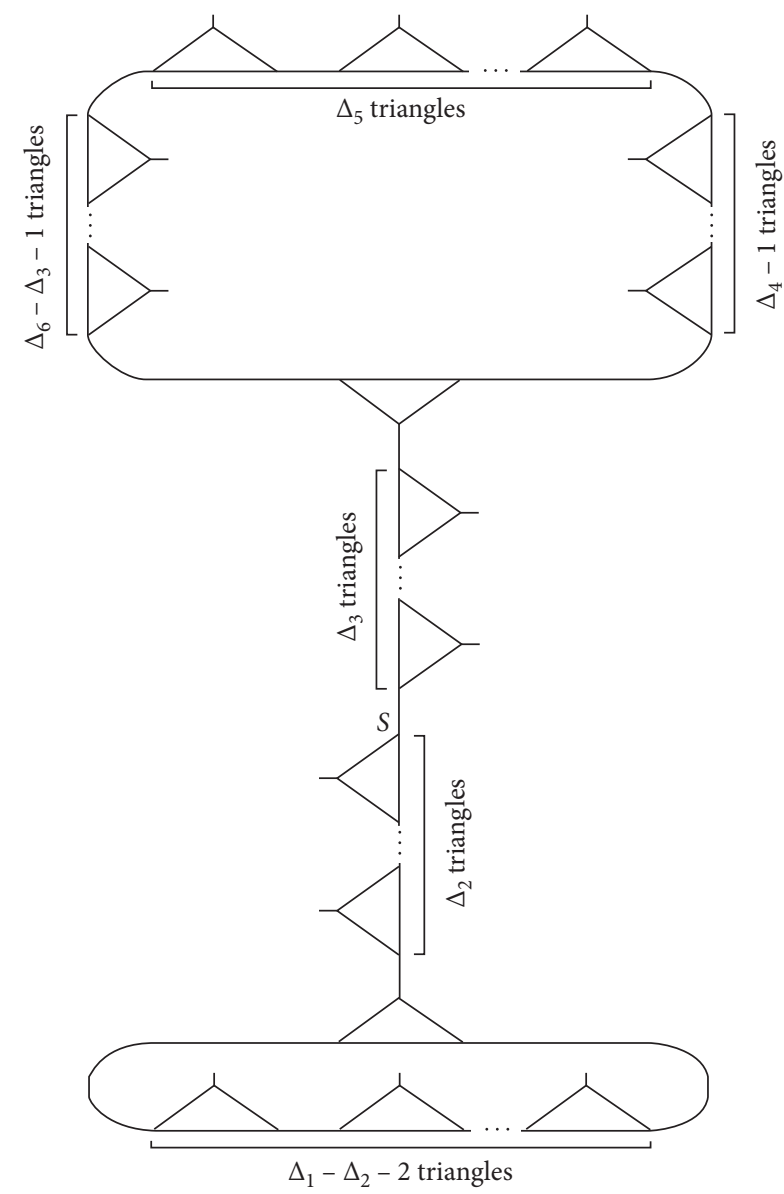

FIgURE 50: Homomorphic image $\Omega_{2}^{32}$.

Let $S^{\prime}$ be the sum of contracted pairs obtained by contracting each element of $\mathrm{S}$, then

$$
\begin{aligned}
S^{\prime}= & 3\left(\Delta_{2}^{2}+3 \Delta_{2}-2\right)+3\left(\Delta_{2}^{2}+3 \Delta_{2}\right)+\frac{3}{2}\left(\Delta_{6}^{2}+3 \Delta_{6}\right)+\frac{3}{2}\left(\Delta_{1}^{2}+3 \Delta_{1}-4\right)+3\left(\Delta_{2}^{2}+3 \Delta_{2}-4\right)+3\left(\Delta_{4}^{2}+3 \Delta_{4}-4\right) \\
& +\frac{3}{2}\left(\Delta_{4}^{2}+3 \Delta_{4}-4\right)+3\left(\Delta_{4}^{2}+3 \Delta_{4}-2\right)+\frac{3}{2}\left(\Delta_{5}^{2}+3 \Delta_{5}\right)+3\left(\Delta_{3}^{2}+3 \Delta_{3}\right)+\frac{3}{2}\left(\Delta_{2}^{2}+3 \Delta_{2}\right)+3\left(\Delta_{3}^{2}+3 \Delta_{3}-2\right) \\
& +3\left(\Delta_{4}^{2}+3 \Delta_{4}-4\right)+3\left(\Delta_{5}^{2}+3 \Delta_{5}\right)+\frac{3}{2}\left(\Delta_{3}^{2}+3 \Delta_{3}-4\right)+6\left(\Delta_{2}+2\right)\left(\Delta_{1}-\Delta_{2}-1\right) \\
& +3\left(\Delta_{4}+2\right)\left(\Delta_{1}-\Delta_{4}-1\right) \\
& +6\left(\Delta_{3}+2\right)\left(\Delta_{2}-\Delta_{3}-1\right) \\
& +6\left(\Delta_{1}-1\right)\left(\Delta_{4}-1\right)+12\left(\Delta_{3}-1\right)\left(\Delta_{4}-1\right)+\sum_{k_{1}=3}^{5}
\end{aligned}
$$



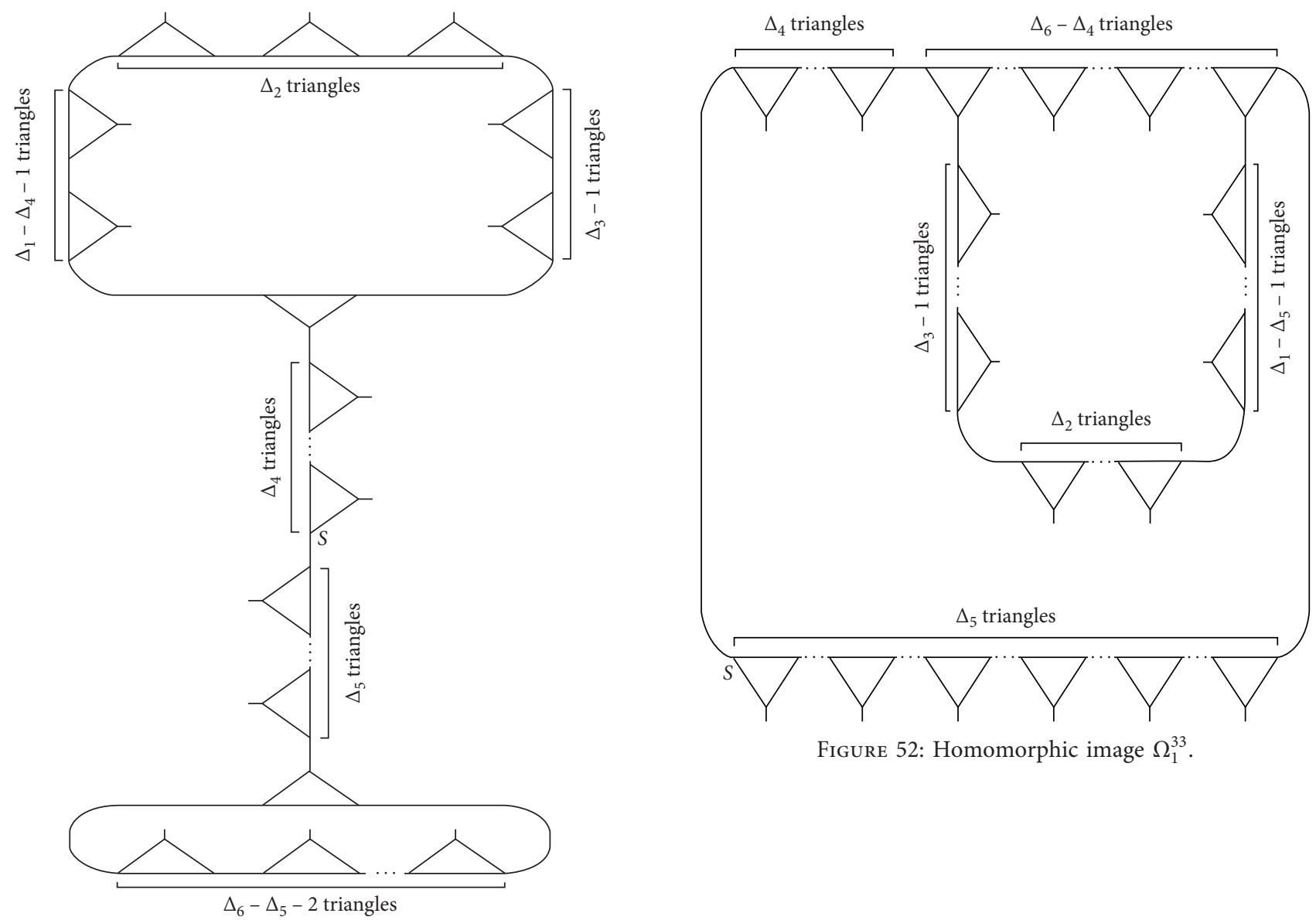

FIgURE 52: Homomorphic image $\Omega_{1}^{33}$.

FIgURE 51: Homomorphic image $\Omega_{4}^{32}$.

$$
\begin{aligned}
& {\left[3\left(\Delta_{k_{1}}^{2}+3 \Delta_{k_{1}}\right)+3\left(\Delta_{k_{1}}^{2}+3 \Delta_{k_{1}}-4\right)+12\left(\Delta_{2}-1\right)\left(\Delta_{k_{1}}-1\right)\right]} \\
& \quad+\sum_{k_{2}=2}^{3}\left[6\left(\Delta_{4}+2\right)\left(\Delta_{k_{2}}-\Delta_{4}-1\right)+12\left(\Delta_{1}-1\right)\left(\Delta_{k_{2}}-1\right)+6\left(\Delta_{k_{2}}-1\right)^{2}+6\left(\Delta_{k_{2}}-1\right)\left(\Delta_{k_{2}}-2\right)+6\left(\Delta_{k_{2}}-1\right)\right] \\
& \quad+\sum_{k_{3}=1}^{2}\left[6\left(\Delta_{5}+2 \Delta_{k_{3}}-\Delta_{5}-1\right)+6\left(\Delta_{4}+\Delta_{5}+2\right)+3\left(\Delta_{k_{3}+1}+2 \Delta_{k_{3}+2}+2\right)\right]+\sum_{k_{4}=1}^{4}\left[3\left(\Delta_{k_{4}}^{2}-3 \Delta_{k_{4}}+2\right)+3\left(\Delta_{k_{4}}-1\right)\right] \\
& \quad+\sum_{k_{5}=2,4}^{2}\left[6\left(\Delta_{k_{5}}+\Delta_{k_{5}+1}+2\right)\right]+\sum_{k_{6}=1,2,5}\left[6\left(\Delta_{k_{6}}+1\right)\right] \\
& \quad=\frac{1}{2}\left[9\left(\Delta_{1}+2 \Delta_{2}+2 \Delta_{3}+\Delta_{4}\right)-3\left(\Delta_{1}+2 \Delta_{2}+2 \Delta_{3}+\Delta_{4}\right)\right]
\end{aligned}
$$

shows that each element of $V(\Delta \times \Delta)$ is contracted.

Now,

$$
\begin{aligned}
|H|= & \Delta_{2}+\Delta_{2}+\Delta_{6}+\left(\Delta_{1}-1\right)+\left(\Delta_{2}-1\right)+\left(\Delta_{4}-1\right)+\left(\Delta_{1}-1\right)+\Delta_{4}+\Delta_{5}+\Delta_{3}+\Delta_{2}+\Delta_{3}+\left(\Delta_{4}-1\right)+\Delta_{5}+\left(\Delta_{3}-1\right) \\
& +\left(\Delta_{1}-\Delta_{2}-1\right)+\frac{\Delta_{1}-\Delta_{4}-\eta_{1}}{2}+\left(\Delta_{2}-\Delta_{3}-1\right)+ \begin{cases}\frac{1}{2}\left(\Delta_{1}-1\right)\left(\Delta_{4}-1\right) & \text { if } \Delta_{4}=1(\bmod 2) \\
\frac{1}{2}\left[\left(\Delta_{1}-1\right)\left(\Delta_{4}-1\right)+1-\eta_{3}\right] & \text { if } \Delta_{4}=0(\bmod 2)\end{cases}
\end{aligned}
$$



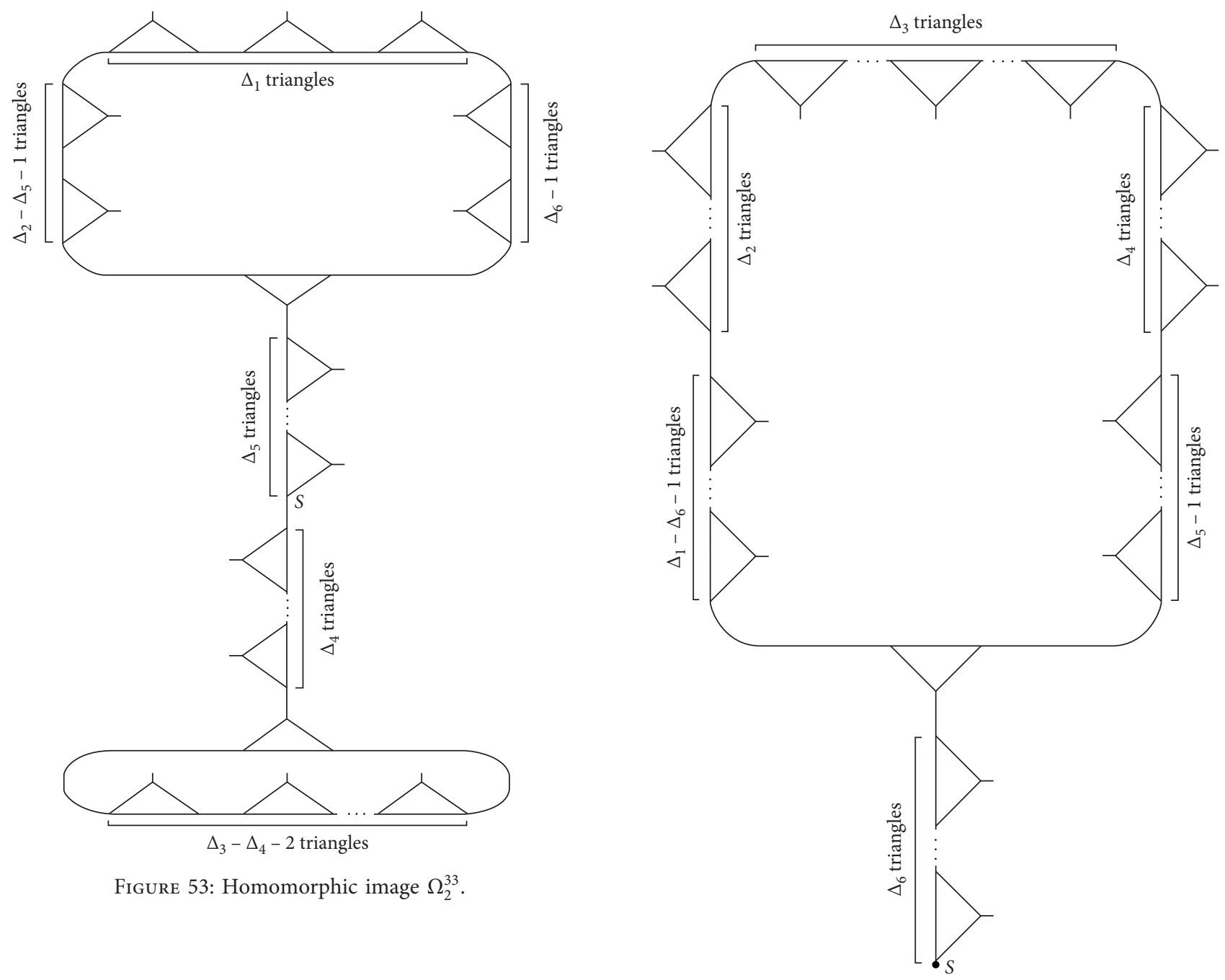

FIGURE 53: Homomorphic image $\Omega_{2}^{33}$.

Figure 54: Homomorphic image $\Omega_{1}^{34}$.

$$
\begin{aligned}
& +\sum_{k_{1}=3}^{5}\left[\Delta_{k_{1}}+\left(\Delta_{k_{1}}-1\right)+\left(\Delta_{2}-1\right)\left(\Delta_{k_{1}}-1\right)\right]+\sum_{k_{1}=3}^{5}\left[\left(\Delta_{k_{2}}-\Delta_{4}-1\right)+\left(\Delta_{1}-1\right)\left(\Delta_{k_{2}}-1\right)+\frac{1}{2} \Delta_{k_{2}}\left(\Delta_{k_{2}}-1\right)+\left(\Delta_{k_{2}}-1\right)\right. \\
& \left.+\frac{1}{2}\left(\Delta_{k_{2}}-1\right)\left(\Delta_{k_{2}}-2\right)\right] \\
& +\sum_{k_{3}=1}^{2}\left[\Delta_{k_{3}}-\Delta_{5}-1+1+1\right]+\sum_{k_{4}=1,4}\left[\frac{1}{4}\left(\Delta_{k_{4}}^{2}-2 \Delta_{k_{4}}+\Delta_{\left(4, k_{4}\right)}\right)+\frac{1}{2}\left(\Delta_{k_{4}}-\eta\left(4, k_{4}\right)\right)\right]+\sum_{k_{5}=2,4}[1]+\sum_{k_{6}=1,2,5}[1] \\
& =\frac{1}{4}\left[\left(\Delta_{1}+2 \Delta_{2}+2 \Delta_{3}+\Delta_{4}\right)^{2}+4\left(\Delta_{1}+2 \Delta_{2}+2 \Delta_{3}+\Delta_{4}\right)+4 \Delta_{4}+\eta\right] .
\end{aligned}
$$




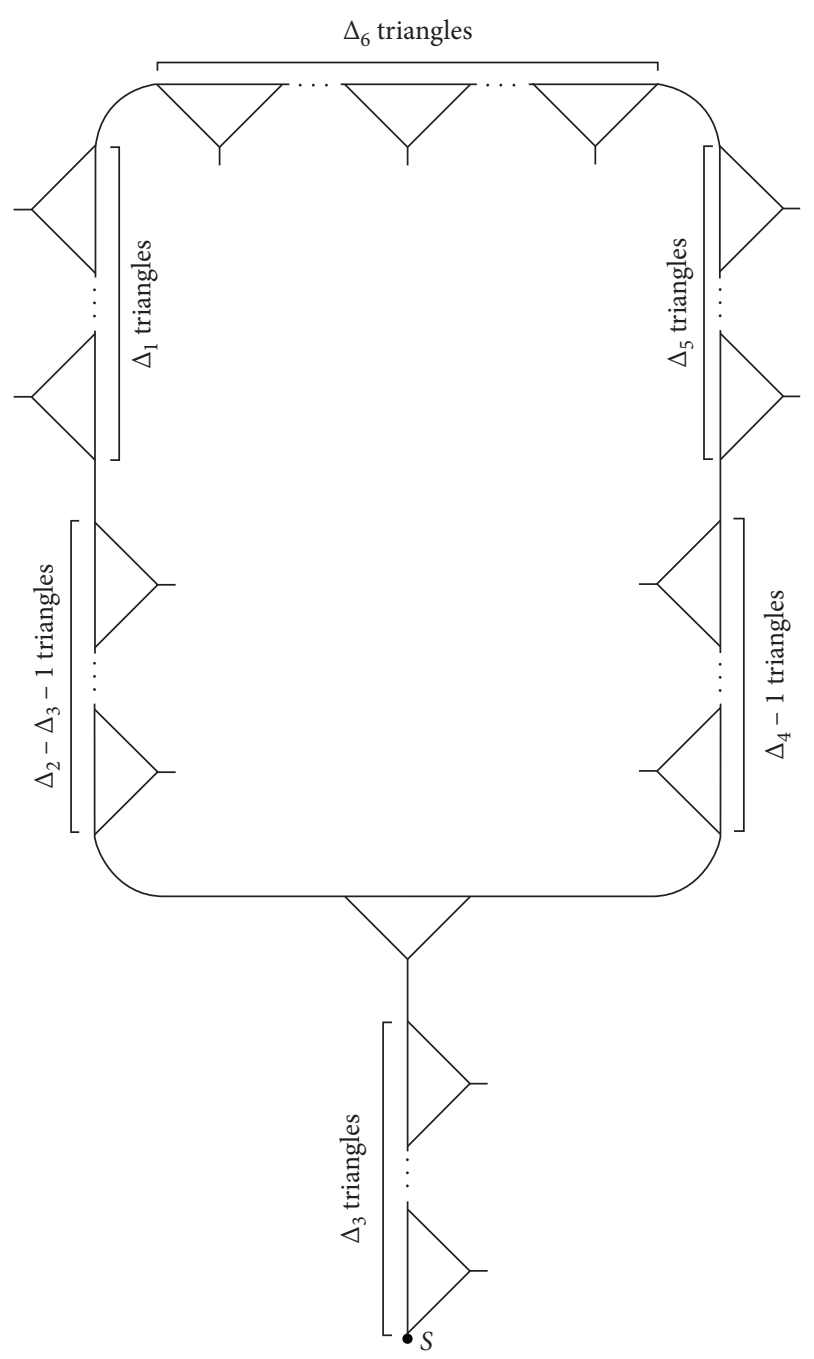

Figure 55: Homomorphic image $\Omega_{2}^{34}$.

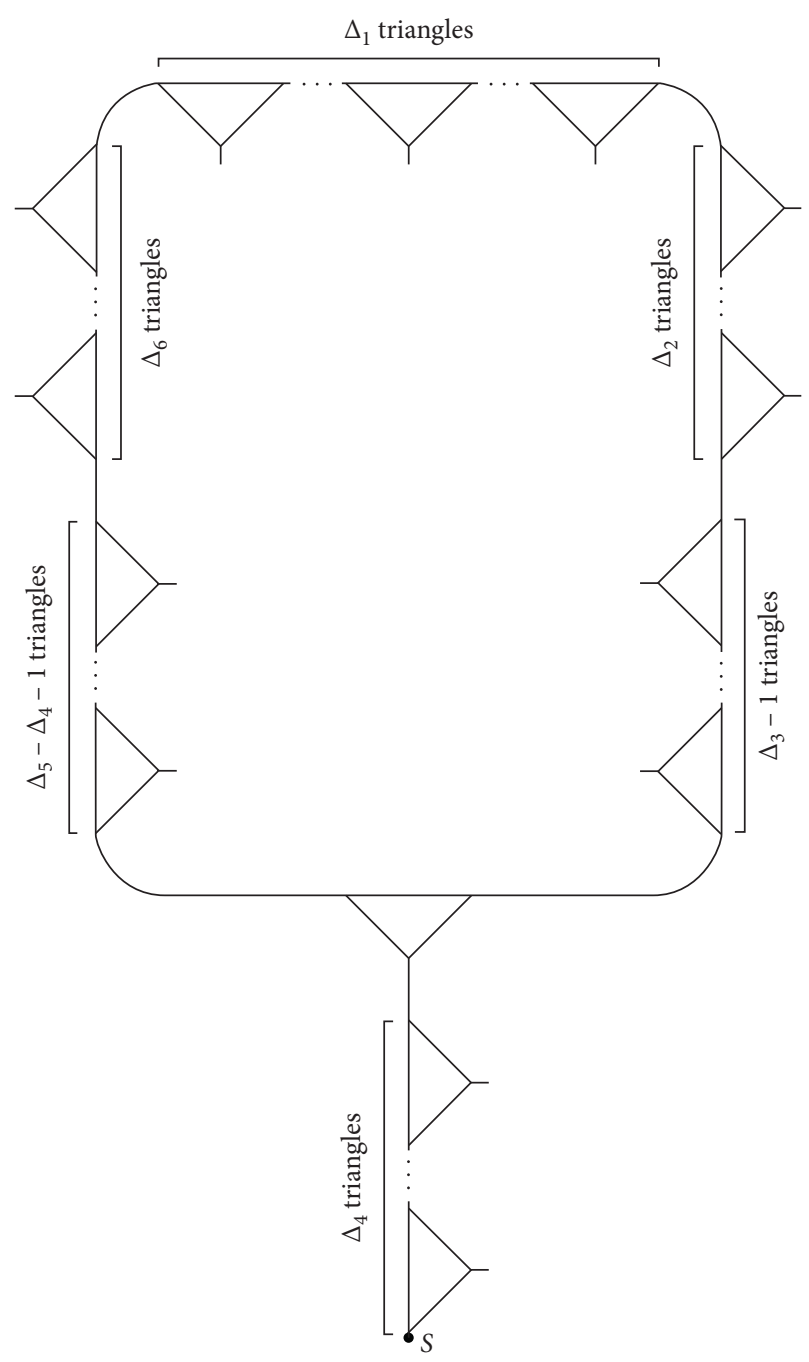

FIGURE 56: Homomorphic image $\Omega_{5}^{34}$. 


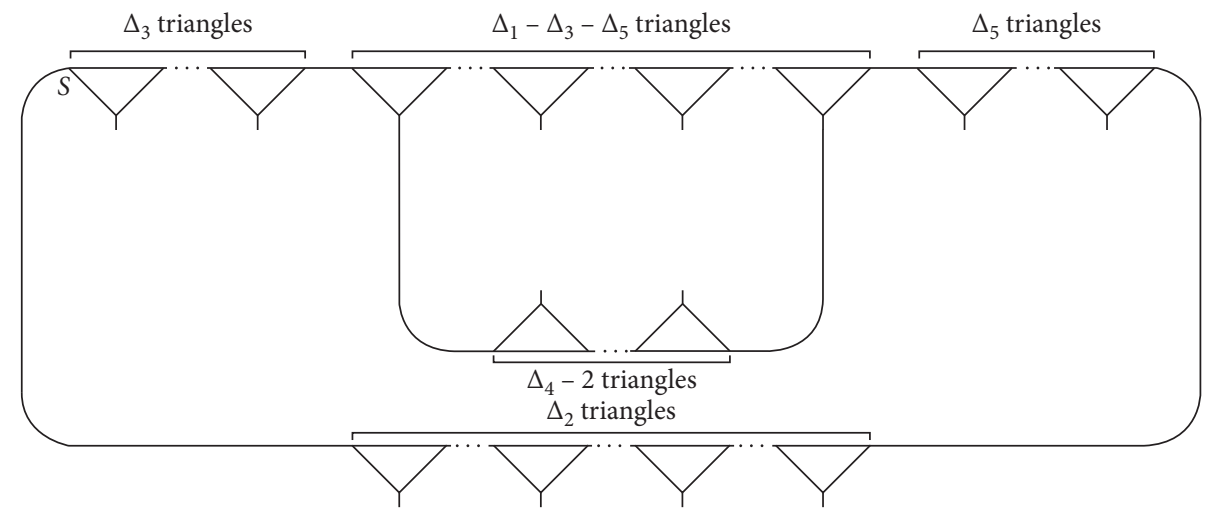

(a)

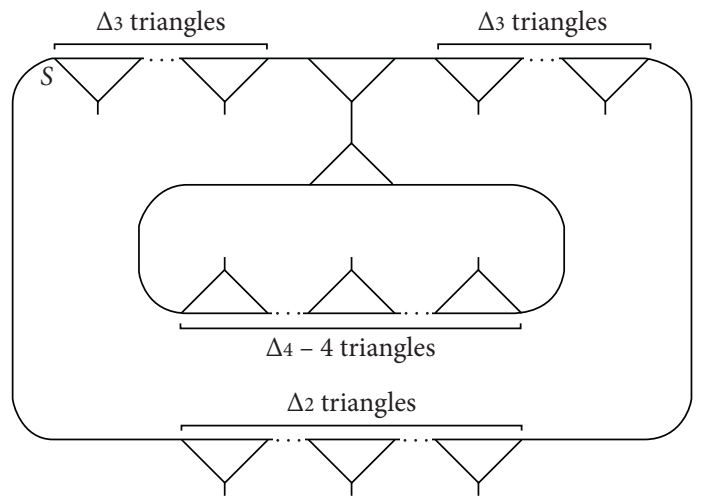

(b)

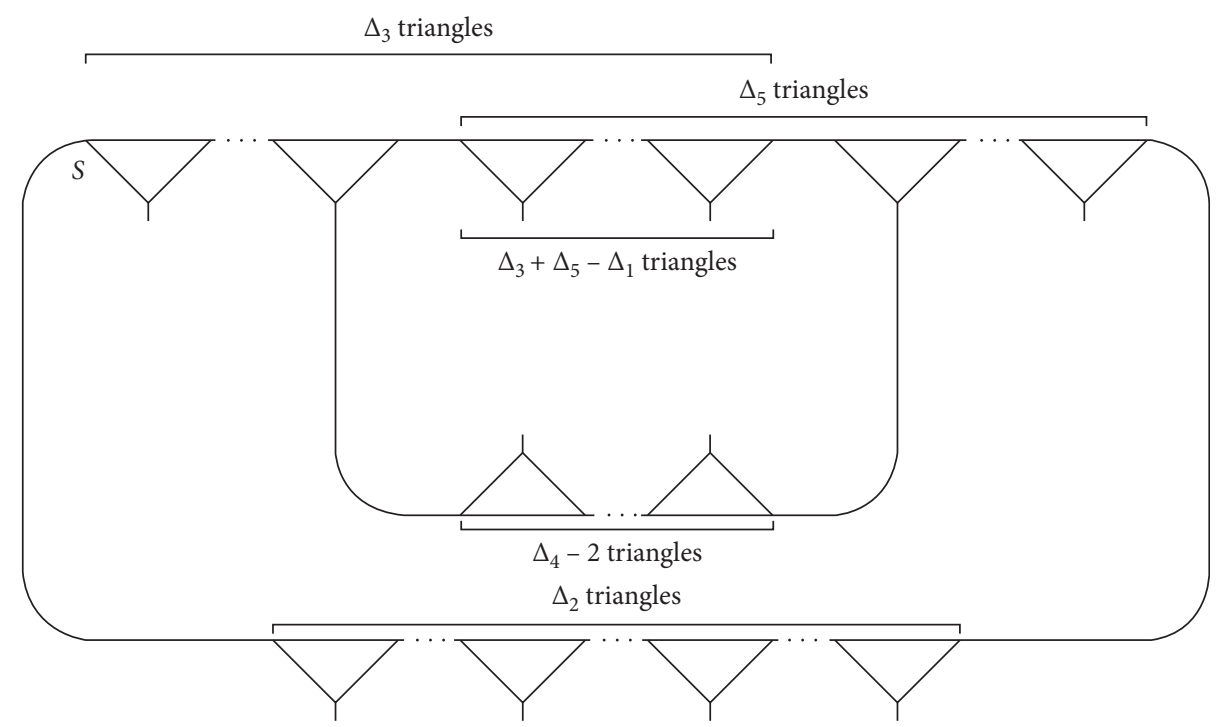

(c)

FIgURE 57: (a) Homomorphic image $\Omega_{1}^{35}: \Delta_{1}>\Delta_{3}+\Delta_{5}+1$. (b) Homomorphic image $\Omega_{1}^{35}: \Delta_{1}=\Delta_{3}+\Delta_{5}+1$. (c) Homomorphic image $\Omega_{1}^{35}$ : $\Delta_{1}<\Delta_{3}+\Delta_{5}+1$. 


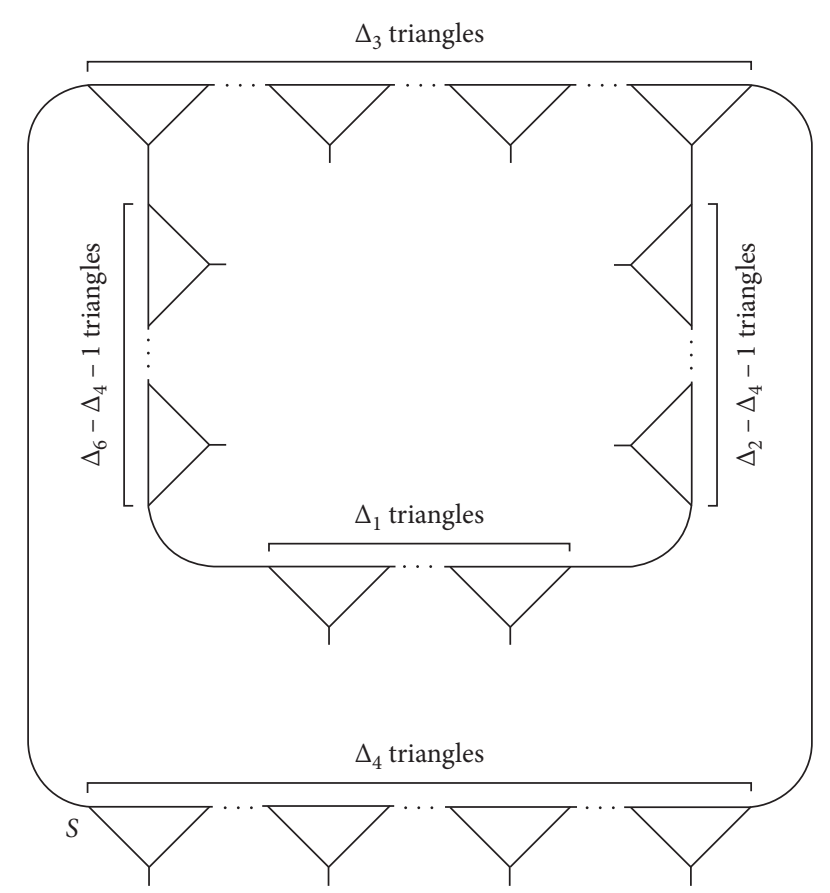

FIgURE 58: Homomorphic image $\Omega_{2}^{35}$.

The value of $|H|$ gives the total number of homomorphic images produced in the contraction of all pairs of vertices of $\Delta$. The value of $S^{\prime}$ guaranteed that the set $H$ contains all homomorphic images.

\section{Conclusion}

Thus, there are total $(1 / 2)\left[9\left(\Delta_{1}+2 \Delta_{2}+2 \Delta_{3}+\Delta_{4}\right)-3\left(\Delta_{1}+\right.\right.$ $\left.\left.2 \Delta_{2}+2 \Delta_{3}+\Delta_{4}\right)\right]$ number of elements in $V(\Delta \times \Delta)$. To find all distinct homomorphic images, we do not need to contract each pair of vertices of $V(\Delta \times \Delta)$. We have to contract only those pairs of vertices, which are in set $S$ and they are $(1 / 4)\left[\left(\Delta_{1}+2 \Delta_{2}+2 \Delta_{3}+\Delta_{4}\right)^{2}+4\left(\Delta_{1}+2 \Delta_{2}+2 \Delta_{3}+\Delta_{4}\right)+\right.$ $\left.4 \Delta_{4}+\eta\right]$ in numbers because if we contract the pair which is not belong to set $S$, we attain the homomorphic image, which we already acquired by contracting the element of set S.

\section{Applications of Homomorphic Copies in Lightweight Cryptography and Chemistry}

The construction of any product by using minimum resources without compromising on quality is primary objective of scientists.

The coset diagrams with 256 vertices are used in the construction of strong $8 \times 8 \mathrm{~S}$-boxes [9]. In present-day block ciphers, cryptographically secure S-boxes are designed to attain the requirements of Shannon's necessity for perplexity. Substitution boxes are the fundamental segments in numerous Feistel network-based block cryptosystems or substitution-permutation (S-P) networks. The use of $8 \times 8$ S-boxes in block cipher is excessively costly. So, it is not surprising that we are seeing strong progress in the field of lightweight cryptography in recent years, for instance
PRESENT. Lightweight cryptographic calculations are utilized in business items, including DESL, PRINTCIPHER, SEA, HIGHT, PRESENT, LED, and KATAN/KTANTAN. For instance, Keeloq is a 32-bit block cipher frequently utilized in the automobile business. Digital signature transponder is a 40-bit block cipher, implemented in wireless authentication systems. Since a homomorphic copy of a graph is smaller graph with the same algebraic structure, it can be quite handy to generate small sized S-boxes by using homomorphic copies of the coset diagram having 256 vertices.

Interrelation of certain types of coset graphs and structure of carbon allotropes not only highlights the connection but also improvises applications in many fields.

Several forms of carbon can be found in nature. One of the most important allotropes of carbon is fullerene which was discovered in 1985. Fullerenes are carbon-cage-like polyhedral molecules in which a large number of carbon atoms are bonded in a nearly spherical symmetric configuration. Fullerenes $C_{n}$ can be drawn for $n=20$ and for all even $n \geq 24$. They have $n$ carbon atoms, $(3 n / 2)$ bonds, 12 pentagonal faces, and $(n / 2)-10$ hexagonal faces. The most important member of the family of fullerenes is $C_{60}$. Fullerenes can be classified in terms of their groups of symmetries. These groups are also known as point groups. Every element of a point group is an isometry of the Euclidian space and so it is either a rotation around an axis or it is a reflection in a plane. The list of all 28 fullerene point groups is: $I_{h}, I, T_{h}, T_{d}, T, D_{6 h}, D_{6 d}, D_{6}, D_{5 h}, D_{5 d}, D_{5}, D_{3 h}, D_{3 d}, D_{3}, D_{2 h}$, $D_{2 d}, D_{2}, S_{6}, S_{4}, C_{3 h}, C_{2 h}, C_{3 v}, C_{3}, C_{2 v}, C_{2}, C_{s}, C_{i}$, and $C_{1}$ [10]. Fullerene $C_{60}$ has icosahedral symmetry, that is, the symmetry group is isomorphic to $A_{5}$. A constructive enumeration of fullerenes has been dealt in detail in [11]. The structure of coset graphs of fullerene $C_{60}$, symmetry group PSL $(2,5)$ and adjacency matrix has been explored through the action of modular group in [12]. Another carbon allotrope with high permutational symmetry is allotrope $D_{168}$ Schwarzite, proposed by Vanderbilt and Tersoff, which has an automorphism group of order 168. In [13], it has been shown that coset diagram for $\operatorname{PSL}(2,7)$ points has interesting relation with carbon allotrope with negative curvature $D_{168}$ Schwarzite. The future studies may extend the present study by investigating the homomorphic copies of the coset graphs for $\operatorname{PSL}(2,5)$ and $\operatorname{PSL}(2,7)$ and their related chemical structures.

\section{Data Availability}

No datasets were generated or analyzed during the current study.

\section{Conflicts of Interest}

The authors declare that they have no conflicts of interest.

\section{References}

[1] M. Akbas, "On suborbital graphs for the modular group," Bulletin of the London Mathematical Society, vol. 33, no. 3, pp. 647-652, 2001. 
[2] Ö. Koruoğlu, "The determination of parabolic points in modular and extended modular groups by continued fractions," Bulletin of the Malaysian Mathematical Sciences Society, vol. 33, no. 3, pp. 439-445, 2010.

[3] Q. Mushtaq, Coset Diagrams for the Modular Group, University of Oxford, Oxford, UK, 1983.

[4] Q. Mushtaq, "Modular group acting on real quadratic fields," Bulletin of the Australian Mathematical Society, vol. 37, no. 2, pp. 303-309, 1988.

[5] M. A. Malik and M. A. Zafar, "Real quadratic irrational numbers and modular group action," Southeast Asian Bulletin of Mathematics, vol. 35, no. 3, pp. 439-445, 2011.

[6] S. M. Husnine, M. A. Malik, and A. Majeed, "On ambiguous numbers of an invariant subset under the action of the modular group St udiaPSL (2, ZZ), "Scientiarum Mathematicarum Hungarica, vol. 42, no. 4, pp. 401-412, 2005.

[7] A. Torstensson, "Coset diagrams in the study of finitely presented groups with an application to quotients of the modular group," Journal of Commutative Algebra, vol. 2, no. 4, pp. 501-514, 2010.

[8] Q. Mushtaq and A. Razaq, "Equivalent pairs of words and points of connection," The Scientific World Journal, vol. 2014, Article ID 505496, 8 pages, 2014.

[9] A. Razaq, A. Yousaf, U. Shuaib, N. Siddiqui, A. Ullah, and A. Waheed, "A novel construction of substitution box involving coset diagram and a bijective map," Security and Communication Networks, vol. 2017, Article ID 5101934, 16 pages, 2017.

[10] P. W. Fowler and D. E. Manolopoulos, "An atlas of fullerenes," Courier Corporation, 2007.

[11] G. Brinkmann and A. W. M. Dress, "A constructive enumeration of fullerenes," Journal of Algorithms, vol. 23, no. 2, pp. 345-358, 1997.

[12] Q. Mushtaq and A. Rafiq, "Adjacency matrices of PSL $(2,5)$ and resemblance of its coset diagrams with fullerene C60," in Academy of Mathematics and Systems Science, Chinese Academy of Sciences, vol. 20, pp. 541-552, , no. No. 04, Suzhou University, Suzhou, China, 2013.

[13] Q. Mushtaq and N. Mumtaz, "PSL $(2,7)$ and carbon allotrope D168 Schwarzite," Journal of Mathematical Chemistry, vol. 56, no. 8, pp. 2485-2494, 2018. 